\title{
Hida families and rational points on elliptic curves
}

\section{Massimo Bertolini ${ }^{1}$, Henri Darmon ${ }^{2}$}

1 Dipartimento di Matematica "Federigo Enriques", Università degli Studi di Milano, Milano, Italy (e-mail: Massimo. Bertolini@mat.unimi.it)

2 Department of Mathematics, McGill University, Burnside Hall, Montreal, QC, Canada (e-mail: darmon@math.mcgill.ca)

Oblatum 7-VI-2006 \& 21-XI-2006

(C) Springer-Verlag 2007

\section{Contents}

\author{
Introduction \\ 1 Modular forms and Hida families \\ 2 Modular forms on quaternion algebras \\ 3 Hida $p$-adic $L$-functions attached to imaginary quadratic fields \\ 4 Heegner points on Shimura curves \\ 5 The main result \\ References
}

\section{Introduction}

Let $E$ be an elliptic curve over $\mathbb{Q}$ of conductor $N=M p$, having a prime $p \| N$ of multiplicative reduction. Because $E$ is modular, it corresponds to a normalised weight two eigenform on $\Gamma_{0}(N)$, whose $q$-expansion is denoted $f=\sum_{n} a_{n} q^{n}$.

Let $\mathcal{X}:=\operatorname{hom}\left(\mathbb{Z}_{p}^{\times}, \mathbb{Z}_{p}^{\times}\right) \simeq \mathbb{Z} /(p-1) \mathbb{Z} \times \mathbb{Z}_{p}$, which contains $\mathbb{Z}$ as a dense subset by associating to $k \in \mathbb{Z}$ the character $x \mapsto x^{k-2}$. Denote by $\mathcal{A}(U)$ the ring of $\mathbb{C}_{p}$-valued $p$-adic analytic functions on a compact open subset $U$ of $\mathcal{X}$. Hida's theory associates to $f$ a neighborhood $U$ of $2 \in \mathcal{X}$ (which can be assumed, for simplicity, to be contained in the residue class of 2 modulo $p-1$ ) and a formal $q$-expansion

$$
f_{\infty}=\sum_{n=1}^{\infty} a_{n}(k) q^{n}, \quad a_{1}=1, \quad a_{n} \in \mathcal{A}(U)
$$


which is characterised by the following properties of its specialisations

$$
f_{k}:=\sum_{n=1}^{\infty} a_{n}(k) q^{n}, \quad(\text { for fixed } k \in U):
$$

(1) For all $k \in U \cap \mathbb{Z}^{\geq 2}$, the series $f_{k}$ is the $q$-expansion of a normalised ordinary eigenform of weight $k$ on $\Gamma_{0}(N)$. (For this reason, $f_{k}$ is sometimes referred to as the "weight $k$ specialisation of $f_{\infty}$ ".)

(2) The weight 2 specialisation $f_{2}$ is equal to $f$.

In [M-Sw-D] and [MTT], a $p$-adic $L$-function $L_{p}(g, s)$ is associated to any ordinary eigenform $g$ of even weight $k \geq 2$, and to a choice of complex period $\Omega_{g}$ attached to $g$. The article [GS] explains how the functions $L_{p}\left(f_{k}, s\right)$ can be packaged into a single two-variable $p$-adic $L$-function: the so-called Mazur-Kitagawa $p$-adic $L$-function, denoted $L_{p}\left(f_{\infty} ; k, s\right)$, whose definition is recalled in Sect. 1 . Section 1 also describes the more general twisted $p$-adic $L$-functions $L_{p}\left(f_{\infty}, \chi, k, s\right)$ attached to a (quadratic) Dirichlet character $\chi$, which similarly interpolate the functions $L_{p}\left(f_{k}, \chi, s\right)$.

The $\mathbb{C}_{p}$-valued function $L_{p}\left(f_{\infty}, k, s\right)$ is $p$-adic analytic in a neighborhood of $(k, s)=(2,1)$. For each $k \in \mathbb{Z}^{\geq 2} \cap U$, it satisfies

$$
L_{p}\left(f_{\infty} ; k, s\right)=\lambda(k) L_{p}\left(f_{k}, s\right),
$$

for a suitable scalar $\lambda(k) \in \mathbb{C}_{p}$ attached to $f_{k}$ satisfying $\lambda(2)=1$. In particular,

$$
L_{p}\left(f_{\infty}, 2, s\right)=L_{p}(f, s)=L_{p}(E, s) .
$$

Among the general properties of $L_{p}\left(f_{k}, s\right)$ studied in [MTT] is a functional equation relating $L_{p}\left(f_{\infty}, k, s\right)$ to $L_{p}\left(f_{\infty}, k, k-s\right)$. The sign that appears in this functional equation does not depend on $k$, and will for that reason be denoted $\operatorname{sign}\left(f_{\infty}\right)$. If $E$ has split multiplicative reduction at $p$, then

$$
\operatorname{sign}\left(f_{\infty}\right)=-\operatorname{sign}(E, \mathbb{Q}),
$$

where $\operatorname{sign}(E, \mathbb{Q})$ denotes the sign in the functional equation of the classical Hasse-Weil $L$-function $L(E, s)$. The discrepancy between the $p$-adic and classical signs reflects the fact that $L_{p}(f, s)$ has a so-called "exceptional zero" in the sense of [MTT] at the central critical point $s=1$, arising from the fact that $p$ is a prime of split multiplicative reduction for $E$.

The present article is primarily interested in the behaviour of $L_{p}\left(f_{\infty}, k, s\right)$ in the presence of such an exceptional zero. One is naturally led to distinguish two cases.

Case 1. $\operatorname{sign}(E, \mathbb{Q})=1$. Equation (3) then shows that $L_{p}\left(f_{\infty} ; k, s\right)$ vanishes identically on the critical line $s=k / 2$, so that

$$
\frac{\partial}{\partial s} L_{p}\left(f_{\infty} ; 2,1\right)=-2 \frac{\partial}{\partial k} L_{p}\left(f_{\infty} ; 2,1\right) .
$$


In [GS], Greenberg and Stevens exploit this identity, together with a factorisation of $L_{p}\left(f_{\infty}, k, s\right)$ when restricted to the line $s=1$, to study the first derivative of $L_{p}(f, s)$ at $s=1$, obtaining:

$$
L_{p}^{\prime}(f, 1)=-2 a_{p}^{\prime}(2) \frac{L(f, 1)}{\Omega_{f}},
$$

$\Omega_{f}$ being the same real period that enters in the definition of $L_{p}(f, s)$. By exploiting the ordinary $\Lambda$-adic representation attached to $f_{\infty}$, and certain properties of its restriction to a decomposition group at $p$ established by Mazur and Wiles, the article [GS] also shows that

$$
-2 a_{p}^{\prime}(2)=\frac{\log (q)}{\operatorname{ord}_{p}(q)}
$$

where $q \in p \mathbb{Z}_{p}$ is Tate's $p$-adic period attached to $E$, which generates the kernel of the Tate uniformisation

$$
\Phi_{\text {Tate }}: \mathbb{C}_{p}^{\times} \longrightarrow E\left(\mathbb{C}_{p}\right) .
$$

Note that formulas (4) and (5) imply that

$$
L_{p}^{\prime}(f, 1)=\frac{\log (q)}{\operatorname{ord}_{p}(q)} \frac{L(f, 1)}{\Omega_{f}},
$$

a statement that was discovered empirically and formulated as the "exceptional zero conjecture" in [MTT].

Case 2. $\operatorname{sign}(E, \mathbb{Q})=-1$. In that case $L(E, 1)=0$, and the Birch and Swinnerton-Dyer conjecture predicts that $E(\mathbb{Q})$ is infinite. The analysis of $L_{p}\left(f_{\infty}, k, s\right)$ at $(k, s)=(2,1)$ carried out by Greenberg and Stevens (and recalled in Sect. 5.2) shows that

$$
\frac{\partial}{\partial s} L_{p}\left(f_{\infty} ; 2,1\right)=\frac{\partial}{\partial k} L_{p}\left(f_{\infty} ; 2,1\right)=0,
$$

so that $L_{p}\left(f_{\infty} ; k, s\right)$ vanishes to order at least 2 at $(k, s)=(2,1)$. On the other hand, because $\operatorname{sign}\left(f_{\infty}\right)=1$, the restriction of $L_{p}\left(f_{\infty} ; k, s\right)$ to the critical line $s=k / 2$ need not vanish identically, and one is led to consider its second derivative.

Let

$$
\log _{E}: E\left(\mathbb{Q}_{p}\right) \longrightarrow \mathbb{G}_{a}\left(\mathbb{Q}_{p}\right)
$$

be the formal group logarithm on $E$, defined by letting $\log _{q}: \mathbb{C}_{p}^{\times} \longrightarrow \mathbb{C}_{p}$ be the branch of the $p$-adic logarithm which satisfies $\log _{q}(q)=0$, and setting

$$
\log _{E}(P):=\log _{q}\left(\Phi_{\text {Tate }}^{-1}(P)\right) .
$$

The main result of this paper, which follows by specialising Theorem 5.4 of Sect. 5.2 to the trivial character, is: 
Theorem 1. Suppose that $E$ has at least two primes of semistable reduction.

(1) There is a global point $\mathbf{P} \in E(\mathbb{Q}) \otimes \mathbb{Q}$ and a scalar $\ell \in \mathbb{Q}^{\times}$such that

$$
\frac{d^{2}}{d k^{2}} L_{p}\left(f_{\infty} ; k, k / 2\right)_{k=2}=\ell \cdot \log _{E}(\mathbf{P})^{2} .
$$

(2) The point $\mathbf{P}$ is of infinite order if and only if $L^{\prime}(E, 1) \neq 0$.

Remark 2. As will be explained shortly, the proof of Theorem 1 exhibits $\mathbf{P}$ as a Heegner point arising from an appropriate Shimura curve parametrisation; Part 2 of Theorem 1 then follows from a result of Zhang [Zh1] extending the Gross-Zagier formula to these points.

Remark 3. Theorem 1 (or rather, the somewhat more general Theorem 5.4) is the main ingredient used in [BD3] to prove the rationality of StarkHeegner points over genus fields of real quadratic fields. Independently of this application, Theorem 1 suggests a general mechanism for producing rational points on elliptic curves from values of $L$-series, encompassing situations where the Heegner point construction used in its proof may be unavailable. (In this connection, it is worth comparing Theorem 1 with the main theorem of $[\mathrm{Ru}]$ concerning elliptic curves with complex multiplication, and with the conjecture of Perrin-Riou described in [PR].) Theorem 1 also yields as a corollary the first non-trivial case of a conjecture of Greenberg [Gre]:

Corollary 4. Assume the hypotheses of Theorem 1. If $L^{\prime}(E, 1) \neq 0$, then there exist infinitely many $k \in \mathbb{Z}^{>2}$ for which $L\left(f_{k}, k / 2\right) \neq 0$.

Remark 5. There would be no difficulty in generalising the statement or the proof of Theorem 1 to the case where $f$ is a normalised eigenform with not necessarily rational Fourier coefficients. In this case the second derivative of $L_{p}\left(f_{\infty} ; k, k / 2\right)$ at $k=2$ is related to the formal group logarithm of a rational point on the modular abelian variety attached to $f$.

Remark 6. The hypothesis of an extra prime of semistable reduction made in Theorem 1 is automatically satisfied if $E$ is semistable everywhere, since a semistable elliptic curve with $\operatorname{sign}(E, \mathbb{Q})=-1$ must have an even number of primes of split multiplicative reduction. We expect Theorem 1 to be true without this assumption, but the proof that we offer relies on it in a crucial way. It would be of interest to dispense with it. This might be possible using Hida families attached to Hilbert modular forms over real quadratic fields.

We now give a brief overview of the proof of Theorem 1. This proof rests on the study of an auxiliary two-variable $p$-adic $L$-function attached to a suitable choice of imaginary quadratic field $K$ in which $p$ is inert. This $L$-function, introduced in Definition 3.5 and Remark 3.6 of Sect. 3 and denoted $L_{p}\left(f_{\infty} / K ; k, s\right)$, interpolates the anticyclotomic $p$-adic $L$-functions attached to $f_{k} / K$ defined in [BD1] and in [BDIS]. Of special interest is its restriction to the central critical line $s=k / 2$, denoted $L_{p}\left(f_{\infty} / K, k\right)$. 
In Sect. 4 a global point $P_{K} \in E(\mathbb{Q}) \otimes \mathbb{Q}$ is constructed as the trace to $\mathbb{Q}$ of a Heegner point attached to $K$ arising from an appropriate Shimura curve parametrisation. Corollary 4.10 of Sect. 4 establishes a direct relationship between $P_{K}$ and the $p$-adic $L$-function $L_{p}\left(f_{\infty} / K, k\right)$, namely,

\section{Theorem 7.}

$$
\frac{d^{2}}{d k^{2}} L_{p}\left(f_{\infty} / K, k\right)_{k=2}=2 \log _{E}\left(P_{K}\right)^{2} .
$$

This result is analogous to Part 1 of Theorem 1, with the crucial difference that the point $P_{K}$ is independently defined via a Heegner-type construction, so that the proof of an identity like (8) can be approached by building on the techniques introduced in [BD2].

Remark 8. It would be worthwhile to understand Theorem 7 in the framework of the $p$-adic Birch and Swinnerton-Dyer conjectures for the anticyclotomic setting, in the same way that the conjectures of [BD1] inspired the main results of [BD2].

Section 3.3 uses work of Gross, Hatcher, Zhang and Hui Xue to describe a precise interpolation property relating $L_{p}\left(f_{\infty} / K, k\right)$ to the algebraic parts of the corresponding classical central critical values $L\left(f_{k} / K, k / 2\right)$ for even integers $k \geq 2$. This leads to the following factorisation formula which is proved in Sect. 5.1:

Theorem 9. Let $\epsilon_{K}$ be the quadratic Dirichlet character associated to $K$. There exists a p-adic analytic function $\eta$ of $k \in U$ satisfying $\eta(2) \in \mathbb{Q}^{\times}$, for which

$$
L_{p}\left(f_{\infty} / K, k\right)=\eta(k) L_{p}\left(f_{\infty}, k, k / 2\right) L_{p}\left(f_{\infty}, \epsilon_{K}, k, k / 2\right),
$$

for all $k \in U$.

The proof of Theorem 1, which is described in Sect. 5, proceeds by invoking non-vanishing results for quadratic twists of $L$-series (such as those that are explained in [MM]) to choose an imaginary quadratic field $K$ in such a way that $L\left(f, \epsilon_{K}, 1\right) \neq 0$. Since $p$ is inert in $K$, we have $\epsilon_{K}(p)=-a_{p}$. Therefore the $p$-adic $L$-function $L_{p}\left(f, \epsilon_{K}, s\right)$ does not have an exceptional zero. In particular, $L_{p}\left(f, \epsilon_{K}, 1\right)$ belongs to $\mathbb{Q}^{\times}$.

Taking second derivatives at $k=2$ in the formula (9) of Theorem 9 and applying Theorem 7 yields

$$
\eta(2) \frac{d^{2}}{d k^{2}} L_{p}\left(f_{\infty} ; k, k / 2\right)_{k=2} \cdot L_{p}\left(f, \epsilon_{K}, 1\right)=2 \log _{E}\left(P_{K}\right)^{2} .
$$

Theorem 1 now follows by setting $\ell=2 \eta(2)^{-1} L_{p}\left(f, \epsilon_{K}, 1\right)^{-1}$ (a non-zero rational number by the choice of $K$ ) and $\mathbf{P}=P_{K}$. Note that both $\mathbf{P}:=P_{K}$ and $\ell$ depend on the choice of $K$ that was made, but the product $\ell \log _{E}(\mathbf{P})^{2}$ is independent of this choice. 
Part 2 of Theorem 1 follows from a result of Zhang generalising the original Gross-Zagier formula, which expresses the height of $P_{K}$ as a multiple of $L^{\prime}(E / K, 1)$ by a non-zero scalar. In particular, because of the non-vanishing of $L\left(E, \epsilon_{K}, 1\right)$, the point $P_{K}$ is of infinite order precisely when $L^{\prime}(E, 1) \neq 0$.

\section{Modular forms and Hida families}

\subsection{Modular symbols. Let}

$$
g=\sum_{n=1}^{\infty} a_{n}(g) q^{n} \in S_{k}\left(\Gamma_{0}(N)\right)
$$

be a normalised cusp form of even weight $k \geq 2$ on $\Gamma_{0}(N)$. The Fourier coefficients $a_{n}(g)$ generate a finite algebraic extension of $\mathbb{Q}$, denoted $K_{g}$. Fix embeddings of $K_{g}$ into both $\mathbb{C}$ and $\mathbb{C}_{p}$.

Let $\mathfrak{P}_{k}=\mathfrak{P}_{k}\left(\mathbb{C}_{p}\right)$ denote the space of homogeneous polynomials in two variables of degree $k-2$ with coefficients in $\mathbb{C}_{p}$. It is equipped with a right action of $\mathbf{G L}_{2}\left(\mathbb{Q}_{p}\right)$ given by the rule

$$
(P \mid \gamma)(x, y):=P(a x+b y, c x+d y), \quad \text { for } \gamma=\left(\begin{array}{ll}
a & b \\
c & d
\end{array}\right) .
$$

Let $V_{k}=V_{k}\left(\mathbb{C}_{p}\right)$ denote the $\mathbb{C}_{p}$-linear dual of $\mathfrak{P}_{k}$, which inherits a left action of $\mathbf{G L}_{2}\left(\mathbb{Q}_{p}\right)$ by the usual rule

$$
(\gamma R)(P)=R(P \mid \gamma)
$$

A modular symbol with values in an abelian group $G$ is a function

$$
I: \mathbb{P}_{1}(\mathbb{Q}) \times \mathbb{P}_{1}(\mathbb{Q}) \longrightarrow G, \quad \text { denoted }(r, s) \mapsto I\{r \rightarrow s\},
$$

satisfying

$$
I\{r \rightarrow s\}+I\{s \rightarrow t\}=I\{r \rightarrow t\}, \quad \text { for all } r, s, t \in \mathbb{P}_{1}(\mathbb{Q}) .
$$

The space of all such modular symbols is denoted $M S(G)$. The group $\mathbf{G L}_{2}(\mathbb{Q})$ acts on $\mathbb{P}_{1}(\mathbb{Q})$ on the left by Möbius transformations in the usual way. Hence if $G$ is equipped with a left action of $\mathbf{G L}_{2}(\mathbb{Q})$, then $M S(G)$ inherits such an action by the rule

$$
(\gamma I)\{r \rightarrow s\}:=\gamma I\left\{\gamma^{-1} r \rightarrow \gamma^{-1} s\right\} .
$$

To the form $g$ is associated the basic $V_{k}(\mathbb{C})$-valued modular symbol $\tilde{I}_{g}$ defined by

$$
\tilde{I}_{g}\{r \rightarrow s\}(P):=2 \pi i \int_{r}^{s} g(z) P(z, 1) d z .
$$

The invariance property of $g$ under the group $\Gamma_{0}(N)$ implies that $\tilde{I}_{g}$ is fixed by the action of this group, i.e.,

$$
\tilde{I}_{g}\left\{\gamma^{-1} r \rightarrow \gamma^{-1} s\right\}(P \mid \gamma)=\tilde{I}_{g}\{r \rightarrow s\}(P), \quad \text { for all } \gamma \in \Gamma_{0}(N) .
$$


The matrix $c=\left(\begin{array}{cc}1 & 0 \\ 0 & -1\end{array}\right)$ induces an involution on $M S_{\Gamma_{0}(N)}\left(V_{k}(\mathbb{C})\right)$, the space of $V_{k}(\mathbb{C})$-valued modular symbols which are fixed by $\Gamma_{0}(N)$. Let $\tilde{I}_{g}^{+}$ and $\tilde{I}_{g}^{-}$denote the plus and minus eigencomponents of $\tilde{I}_{g}$ for this involution.

The modular symbols $\tilde{I}_{g}, \tilde{I}_{g}^{+}$and $\tilde{I}_{g}^{-}$encode certain complex line integrals of $g$. The following result of Shimura asserts that these functions can be rescaled so as to belong to $M S\left(V_{k}\left(K_{g}\right)\right)$.

Proposition 1.1. There exist complex periods $\Omega_{g}^{+}$and $\Omega_{g}^{-}$with the property that the modular symbols

$$
I_{g}^{+}:=\left(\Omega_{g}^{+}\right)^{-1} \tilde{I}_{g}^{+}, \quad I_{g}^{-}:=\left(\Omega_{g}^{-}\right)^{-1} \tilde{I}_{g}^{-}
$$

belong to $\operatorname{MS}\left(V_{k}\left(K_{g}\right)\right)$. These periods can be chosen to satisfy

$$
\Omega_{g}^{+} \Omega_{g}^{-}=\langle g, g\rangle,
$$

where $\langle g, g\rangle$ is the Petersson scalar product of $g$ with itself.

In Proposition 1.1, the Petersson scalar product is normalised so that, if $g$ and $h$ are cusp forms of weight $k$ on $\Gamma_{0}(M)$, then

$$
\langle g, h\rangle=4 \pi^{2} \iint_{\Gamma_{0}(M) \backslash \mathscr{H}} g(z) \bar{h}(z) y^{k-2} d x d y, \quad z=x+i y,
$$

where the integral is taken over any fundamental region for the action of $\Gamma_{0}(M)$ on $\mathscr{H}$.

Choose a "sign at infinity" $w_{\infty} \in\{+1,-1\}$, and set

$$
\Omega_{g}:=\left\{\begin{array}{ll}
\Omega_{g}^{+} & \text {if } w_{\infty}=+1 ; \\
\Omega_{g}^{-} & \text {if } w_{\infty}=-1 ;
\end{array} \quad I_{g}:= \begin{cases}I_{g}^{+} & \text {if } w_{\infty}=+1 \\
I_{g}^{-} & \text {if } w_{\infty}=-1 .\end{cases}\right.
$$

Note that the modular symbol $I_{g}$ can be viewed as an element of $M S\left(V_{k}\left(\mathbb{C}_{p}\right)\right)$ thanks to the chosen embedding of $K_{g}$ into $\mathbb{C}_{p}$. This will be frequently done in the sequel.

It is useful to record, for future reference, the explicit formula describing the action of the Hecke operator $U_{p}$ on the element $I_{g} \in M S_{\Gamma_{0}(N)}\left(V_{k}\left(\mathbb{C}_{p}\right)\right)$. For each $0 \leq a \leq p-1$, denote by $\gamma_{a}$ the matrix $\left(\begin{array}{ll}1 & a \\ 0 & p\end{array}\right)$.

Lemma 1.2. For all $r, s \in \mathbb{P}_{1}(\mathbb{Q})$ and all $P \in \mathfrak{P}_{k}\left(\mathbb{C}_{p}\right)$,

$$
I_{g \mid U_{p}}\{r \rightarrow s\}(P)=\sum_{a=0}^{p-1} I_{g}\left\{\gamma_{a} r \rightarrow \gamma_{a} s\right\}\left(P \mid p \gamma_{a}^{-1}\right) .
$$

Proof. This follows (after a direct calculation) from the proposition in $\S 4$, Chap. I of [MTT]. 
The modular symbol $I_{g}$ can be used to define a function of $j, a, m \in \mathbb{Z}$ and $P \in \mathfrak{P}_{k}\left(\mathbb{C}_{p}\right)$ by the rule

$$
I_{g}[j, a, m]:=I_{g}\left\{\infty \rightarrow \frac{a}{m}\right\}\left(\left(x-\frac{a}{m} y\right)^{j-1} y^{k-j-1}\right) .
$$

The invariance properties of $I_{g}$ under the matrix $\left(\begin{array}{ll}1 & 1 \\ 0 & 1\end{array}\right)$ imply that the value of $I_{g}[j, a, m]$ depends only on the value of $a$ in $\mathbb{Z} / m \mathbb{Z}$.

Let $\chi:(\mathbb{Z} / m \mathbb{Z})^{\times} \longrightarrow\{ \pm 1\}$ be a primitive quadratic Dirichlet character, and let

$$
\tau(\chi):=\sum_{a=1}^{m} \chi(a) e^{2 \pi i a / m}
$$

denote the usual Gauss sum. The following proposition relates $I_{g}$ to special values of $L$-functions, and in particular asserts the algebraicity of these special values.

Proposition 1.3. Let $1 \leq j \leq k-1$ be an integer, and suppose that the character $\chi$ satisfies $\chi(-1)=(-1)^{j-1} w_{\infty}$. Then the expression

$$
L^{*}(g, \chi, j):=\frac{(j-1) ! \tau(\chi)}{(-2 \pi i)^{j-1} \Omega_{g}} L(g, \chi, j)
$$

belongs to $K_{g}$, and

$$
L^{*}(g, \chi, j)=\sum_{a=1}^{m} \chi(a) I_{g}[j, a, m] .
$$

Proof. This well known formula of Birch and Manin expressing special values of $L$-series in terms of modular symbols can be found, for example, in formula (8.6) of [MTT].

The expression $L^{*}(g, \chi, j)$ will sometimes be referred to as the algebraic part of the special value $L(g, \chi, j)$. Note that this expression is a multiple of $L(g, \chi, j)$ by a simple non-zero factor, and that it can be viewed as an element of $\mathbb{C}_{p}$ thanks to the chosen embedding of $K_{g}$ into $\mathbb{C}_{p}$.

\subsection{Hida theory. Let}

$$
\tilde{\Lambda}=\mathbb{Z}_{p}\left[\left[\mathbb{Z}_{p}^{\times}\right]\right]:=\lim _{\leftarrow} \mathbb{Z}_{p}\left[\left(\mathbb{Z} / p^{n} \mathbb{Z}\right)^{\times}\right], \quad \Lambda=\mathbb{Z}_{p}\left[\left[\left(1+p \mathbb{Z}_{p}\right)^{\times}\right]\right]
$$

denote the usual Iwasawa algebras. It is often useful to view $\tilde{\Lambda}$ as functions on the space of continuous $\mathbb{Z}_{p}$-algebra homomorphisms

$$
\mathcal{X}:=\operatorname{hom}_{\mathrm{cts}}\left(\tilde{\Lambda}, \mathbb{Z}_{p}\right)=\operatorname{hom}_{\mathrm{gp}}\left(\mathbb{Z}_{p}^{\times}, \mathbb{Z}_{p}^{\times}\right) \simeq \mathbb{Z} /(p-1) \mathbb{Z} \times \mathbb{Z}_{p} .
$$


Given $\lambda \in \tilde{\Lambda}$, and $x \in \mathcal{X}$, we therefore occasionally write $\lambda(x)$ for $x(\lambda)$. (Elements of $\tilde{\Lambda}$ are then referred to as Iwasawa functions on $\mathcal{X}$.)

The set $\mathbb{Z}$ of integers embeds naturally in $\mathcal{X}$ by the rule

$$
k \mapsto x_{k}, \quad \text { with } x_{k}(t)=t^{k-2} \text { for } t \in \mathbb{Z}_{p}^{\times} .
$$

Note that with these conventions, the element 2 corresponds to the augmentation map on $\tilde{\Lambda}$ and $\Lambda$.

For each integer $n \geq 0$, let $Y\left(N, p^{n}\right)$ denote the open modular curve whose complex points are identified with $\mathscr{H} /\left(\Gamma_{0}(N) \cap \Gamma_{1}\left(p^{n}\right)\right)$, and let

$$
H_{n}:=H_{1}\left(Y\left(N, p^{n}\right), \mathbb{Z}_{p}\right)=\left(\Gamma_{0}(N) \cap \Gamma_{1}\left(p^{n}\right)\right)_{\mathrm{ab}} \otimes \mathbb{Z}_{p} .
$$

The $H_{n}$ are finitely generated $\mathbb{Z}_{p}$-modules, and are equipped with a natural action of the Hecke operators $T_{\ell}$ (for $\ell \neq p$ ) as well as of the operator $T_{p}=U_{p}$. We may decompose $H_{n}$ as a direct sum of two spaces

$$
H_{n}=H_{n}^{\text {ord }} \oplus H_{n}^{\text {nil }},
$$

on which the Hecke operator $U_{p}$ acts invertibly and topologically nilpotently, respectively. More precisely,

$$
H_{n}^{\text {ord }}:=e_{\text {ord }} H_{n},
$$

where $e_{\text {ord }}:=\lim _{n \longrightarrow \infty} U_{p}^{n !}$ is Hida's projector to the ordinary part in the Hecke algebra.

The natural homomorphisms $H_{n+1} \longrightarrow H_{n}$ induced by the inclusions

$$
\Gamma_{0}(N) \cap \Gamma_{1}\left(p^{n+1}\right) \longrightarrow \Gamma_{0}(N) \cap \Gamma_{1}\left(p^{n}\right)
$$

respect the ordinary parts, and hence the modules $H_{n}^{\text {ord }}$ are part of a projective system of modules over the Hecke algebra. Note also that the diamond operators give rise to an inclusion of the group ring $\mathbb{Z}_{p}\left[\left(\mathbb{Z} / p^{n} \mathbb{Z}\right)^{\times}\right]$in the ring of Hecke operators acting on $H_{n}$. The inverse limit

$$
H_{\infty}^{\text {ord }}:=\lim _{\leftarrow} H_{n}^{\text {ord }}
$$

therefore inherits a structure of a $\Lambda$-module on which the Hecke operators act $\Lambda$-linearly. Let $\mathbb{T}_{\infty}^{\text {ord }}$ denote the $\Lambda$-algebra generated by the images of the Hecke operators acting on $H_{\infty}^{\text {ord }}$.

Crucial to the constructions of this paper is the following structure theorem due to Hida.

Theorem 1.4 (Hida). The algebra $\mathbb{T}_{\infty}^{\text {ord }}$ is a free $\Lambda$-module of finite rank, and is unramified over the augmentation ideal of $\Lambda$. 
The normalised eigenform $f$ of the introduction gives rise to an algebra homomorphism

$$
\eta_{f}: \mathbb{T}_{\infty}^{\text {ord }} \longrightarrow \mathbb{Z}_{p}
$$

sending the Hecke operator $T_{n}$ to $a_{n}(f)$, and $U_{p}$ to $a_{p}(f)$. The restriction of $\eta_{f}$ to $\Lambda$ is the augmentation map, corresponding to $2 \in \mathcal{X}$. Let $\Lambda^{\dagger} \supset \Lambda$ denote the ring of power series with coefficients in $\mathbb{C}_{p}$ which converge in some neighborhood of $2 \in \mathcal{X}$. Since $\Lambda^{\dagger}$ is Henselian and since the augmentation ideal is unramified in $\mathbb{T}_{\infty}^{\text {ord }}$, the homomorphism $\eta_{f}$ lifts uniquely to a $\Lambda$-algebra homomorphism

$$
\eta_{f_{\infty}}: \mathbb{T}_{\infty}^{\text {ord }} \longrightarrow \Lambda^{\dagger}
$$

Following the notations of the introduction, let $a_{n}(k):=\eta_{f_{\infty}}\left(T_{n}\right)$. The formal $q$-expansion with coefficients in $\Lambda^{\dagger}$

$$
f_{\infty}:=\sum_{n=1}^{\infty} a_{n}(k) q^{n}
$$

is the Hida family alluded to in the introduction. Fix a neighborhood $U$ of $2 \in \mathcal{X}$ on which the elements $a_{n}$ converge, and suppose for simplicity that $U$ is contained in the residue class of 2 modulo $p-1$.

If $k$ belongs to $U \cap \mathbb{Z}^{\geq 2}$, the weight $k$ specialisation

$$
f_{k}:=\sum_{n} a_{n}(k) q^{n}
$$

is a normalised eigenform of weight $k$ on $\Gamma_{0}(N)$. It is new at the primes dividing $M=N / p$ (see for example Theorem (2.6) of [GS]), but it is not new at $p$ when $k>2$. More precisely, if $k$ belongs to $U \cap \mathbb{Z}^{>2}$, the modular form $f_{k}$ arises from a normalised eigenform on $\Gamma_{0}(M)$, denoted $f_{k}^{\sharp}=\sum_{n} a_{n}\left(f_{k}^{\sharp}\right) q^{n}$. If $(p, n)=1$, then $a_{n}\left(f_{k}^{\sharp}\right)=a_{n}\left(f_{k}\right)$. Letting

$$
1-a_{p}\left(f_{k}^{\sharp}\right) p^{-s}+p^{k-1-2 s}=\left(1-\alpha_{p}(k) p^{-s}\right)\left(1-\beta_{p}(k) p^{-s}\right)
$$

denote the Euler factor at $p$ that appears in the $L$-series of $f_{k}^{\sharp}$, we may order the roots $\alpha_{p}(k)$ and $\beta_{p}(k)$ in such a way that

$$
\alpha_{p}(k)=a_{p}\left(f_{k}\right), \quad \beta_{p}(k)=p^{k-1} a_{p}\left(f_{k}\right)^{-1} .
$$

With this convention, we have

$$
f_{k}(z)=f_{k}^{\sharp}(z)-\beta_{p}(k) f_{k}^{\sharp}(p z) .
$$

The field $K_{f_{k}}$ generated by the Fourier coefficients of $f_{k}$ is an extension of $\mathbb{Q}$ of finite degree, which we view as being embedded into both $\mathbb{C}$ and $\mathbb{C}_{p}$. 
For each $k$, we choose the Shimura periods $\Omega_{k}^{+}:=\Omega_{f_{k}}^{+}$and $\Omega_{k}^{-}:=\Omega_{f_{k}}^{-}$as in Proposition 1.1, requiring that

$$
\Omega_{2}^{+} \Omega_{2}^{-}=\langle f, f\rangle, \quad \Omega_{k}^{+} \Omega_{k}^{-}=\left\langle f_{k}^{\sharp}, f_{k}^{\sharp}\right\rangle(k>2) .
$$

Thanks to these periods we may talk about the $V_{k}\left(\mathbb{C}_{p}\right)$-valued modular symbols $I_{f_{k}}^{+}$and $I_{f_{k}}^{-}$associated to each $f_{k}$. Since the Fourier coefficients of $f_{k}$ vary $p$-adically analytically with $k$, it is natural to ask whether the functions $I_{f_{k}}$, which encode the Shimura periods of $f_{k}$, can be likewise viewed as part of an analytically varying family (as a function of $k$ ). This question is considered in the next section.

1.3. Measure-valued modular symbols. Let $L_{*}:=\mathbb{Z}_{p}^{2}$ denote the standard $\mathbb{Z}_{p}$-lattice in $\mathbb{Q}_{p}^{2}$, and let $L_{*}^{\prime}$ denote its set of primitive vectors, i.e., the vectors in $L_{*}$ which are not divisible by $p$.

The space of continuous $\mathbb{C}_{p}$-valued functions on $L_{*}^{\prime}$ is equipped with the right action of $\mathbf{G L}_{2}\left(\mathbb{Z}_{p}\right)$ defined by the rule which is compatible with (10)

$$
(F \mid g)(x, y):=F(a x+b y, c x+d y), \quad \text { for } g=\left(\begin{array}{ll}
a & b \\
c & d
\end{array}\right),
$$

and with a topology given by the sup norm. Its continuous dual is called the space of measures on $L_{*}^{\prime}$. Denote by $\mathbb{D}_{*}$ this latter space. The action of the group $\mathbb{Z}_{p}^{\times}$on $L_{*}^{\prime}$ given by $\lambda(x, y)=(\lambda x, \lambda y)$ gives rise to a natural $\tilde{\Lambda}$-module structure on $\mathbb{D}_{*}$ by setting

$$
\int_{L_{*}^{\prime}} F(x, y) d(a \cdot \mu)(x, y):=\int_{L_{*}^{\prime}} F(a x, a y) d \mu(x, y),
$$

for all $a \in \mathbb{Z}_{p}^{\times}$. If $X$ is any compact open subset of $L_{*}^{\prime}$, we adopt the common notation

$$
\int_{X} F d \mu:=\int_{L_{*}^{\prime}} \mathbf{1}_{X} F d \mu
$$

where $\mathbf{1}_{X}$ is the characteristic function of $X$. Note that the group $\mathbf{G L}_{2}\left(\mathbb{Z}_{p}\right)$ also acts on $\mathbb{D}_{*}$ on the left by translation, so that

$$
\int_{X} F d(\gamma \mu)=\int_{\gamma^{-1} X}(F \mid \gamma) d \mu .
$$

Denote by $\Gamma_{0}\left(p \mathbb{Z}_{p}\right)$ the group of matrices in $\mathbf{G L}_{2}\left(\mathbb{Z}_{p}\right)$ which are upper triangular modulo $p$. Our interest in the space $\mathbb{D}_{*}$ lies in the fact that it is equipped, for all $k \in \mathbb{Z}^{\geq 2}$, with a $\Gamma_{0}\left(p \mathbb{Z}_{p}\right)$-equivariant homomorphism

$$
\rho_{k}: \mathbb{D}_{*} \longrightarrow V_{k}
$$


defined by

$$
\rho_{k}(\mu)(P):=\int_{\mathbb{Z}_{p} \times \mathbb{Z}_{p}^{\times}} P(x, y) d \mu(x, y) .
$$

(Note that $\rho_{k}$ does not respect the full action of $\mathbf{G L}_{2}\left(\mathbb{Z}_{p}\right)$, because the domain of integration that appears in its definition is only preserved by $\Gamma_{0}\left(p \mathbb{Z}_{p}\right)$.) The homomorphism $\rho_{k}$ gives rise to a homomorphism, denoted by the same letter by abuse of notation:

$$
\rho_{k}: M S_{\Gamma_{0}(M)}\left(\mathbb{D}_{*}\right) \longrightarrow M S_{\Gamma_{0}(N)}\left(V_{k}\right) .
$$

Recall the ring $\Lambda^{\dagger} \supset \Lambda$ of power series which converge in some neighborhood of $2 \in \mathcal{X}$, and set

$$
\mathbb{D}_{*}^{\dagger}:=\mathbb{D}_{*} \otimes_{\Lambda} \Lambda^{\dagger}
$$

If

$$
\mu=\lambda_{1} \mu_{1}+\cdots+\lambda_{t} \mu_{t}, \quad \text { with } \lambda_{j} \in \Lambda^{\dagger}, \quad \mu_{j} \in \mathbb{D}_{*}
$$

is any element of $\mathbb{D}_{*}^{\dagger}$, then there exists a neigbourhood $U_{\mu}$ of $2 \in X$ on which all the coefficients $\lambda_{j}$ converge. Call such a region $U_{\mu}$ a neighborhood of regularity for $\mu$.

Given $k \in U_{\mu}$, a continuous function $F$ on $L_{*}^{\prime}$ is said to be homogeneous of degree $k-2$ if $F(\lambda x, \lambda y)=\lambda^{k-2} F(x, y)$, for all $\lambda \in \mathbb{Z}_{p}^{\times}$. For any $k \in U_{\mu}$, and any homogenous function $F(x, y)$ of degree $k-2$, the function $F$ can be integrated against $\mu$ by the rule

$$
\int_{X} F d \mu:=\lambda_{1}(k) \int_{X} F d \mu_{1}+\cdots+\lambda_{t}(k) \int_{X} F d \mu_{t},
$$

for any compact open $X \subset L_{*}^{\prime}$.

The space $M S_{\Gamma_{0}(M)}\left(\mathbb{D}_{*}\right)$ is equipped with a natural action of the Hecke operators, including an operator $U_{p}$ which is defined so as to be compatible with the specialisation maps $\rho_{k}$ of (17). More precisely, in the notations of Lemma 1.2, it is given by the formula

$$
\int_{X} F d\left(U_{p} \mu\right)\{r \rightarrow s\}=\sum_{a=0}^{p-1} \int_{p^{-1} \gamma_{a} X}\left(F \mid p \gamma_{a}^{-1}\right) d \mu\left\{\gamma_{a} r \rightarrow \gamma_{a} s\right\} .
$$

Let $M S_{\Gamma_{0}(M)}^{\text {ord }}\left(\mathbb{D}_{*}\right)$ denote the ordinary subspace of $M S_{\Gamma_{0}(M)}\left(\mathbb{D}_{*}\right)$. Proposition (6.1) of [GS] asserts that this module is free and of finite rank over $\Lambda$. Therefore the same is true of the $\Lambda^{\dagger}$-module

$$
M S_{\Gamma_{0}(M)}^{\text {ord }}\left(\mathbb{D}_{*}\right)^{\dagger}:=M S_{\Gamma_{0}(M)}^{\text {ord }}\left(\mathbb{D}_{*}\right) \otimes_{\Lambda} \Lambda^{\dagger} \subset M S_{\Gamma_{0}(M)}\left(\mathbb{D}_{*}^{\dagger}\right) .
$$

Given $r, s \in \mathbb{P}_{1}(\mathbb{Q})$, and $\mu \in M S_{\Gamma_{0}(M)}^{\text {ord }}\left(\mathbb{D}_{*}\right)^{\dagger}$, denote by $\mu\{r \rightarrow s\}$ the cor- 
responding element of $\mathbb{D}_{*}^{\dagger}$. It is possible to choose a common neighborhood of regularity $U_{\mu}$ for the measures $\mu\{r \rightarrow s\}$, and this makes it possible to define $\rho_{k}(\mu)$ for all $k \in U_{\mu} \cap \mathbb{Z}^{\geq 2}$.

Recall the Hida family $f_{\infty}$ that was introduced in the previous section. The following result of Greenberg and Stevens plays a key role in the constructions of this section.

Theorem 1.5. There exists a neighborhood $U$ of $2 \in X$ and a measurevalued symbol $\mu_{*} \in M S_{\Gamma_{0}(M)}^{\text {ord }}\left(\mathbb{D}_{*}\right)^{\dagger}$ which is regular on $U$, and satisfies

(1) $\rho_{2}\left(\mu_{*}\right)=I_{f}$;

(2) For all $k \in U \cap \mathbb{Z}^{\geq 2}$, there exists a scalar $\lambda(k) \in \mathbb{C}_{p}$ such that

$$
\rho_{k}\left(\mu_{*}\right)=\lambda(k) I_{f_{k}} .
$$

Proof. See Theorem (5.13) of [GS], whose proof is explained in Sect. 6 of that paper.

Remark 1.6. Note that $\mu_{*}$ depends on the choice of sign $w_{\infty}$, and that there are therefore two $\mathbb{D}_{*}^{\dagger}$-valued modular symbols, $\mu_{*}^{+}$and $\mu_{*}^{-}$, which interpolate the modular symbols $I_{f_{k}}^{+}$and $I_{f_{k}}^{-}$respectively. Likewise, there are two families of scalars, $\lambda^{+}(k)$ and $\lambda^{-}(k)$, which depend on the choices of Shimura periods $\Omega_{k}^{+}$and $\Omega_{k}^{-}$that had to be made for each $f_{k}$ in order to define $I_{f_{k}}^{+}$and $I_{f_{k}}^{-}$respectively. There has been no attempt at making a coherent choice of these Shimura periods as $k$ varies (and it is not even clear that such a choice can be made in a natural way) so that the function $\lambda(k)$ cannot be expected to be continuous as a function of $k$ (with respect to the topology on $\mathbb{Z}^{\geq 2}$ induced by its inclusion into $\mathcal{X}$ ). It would be interesting to know whether it is possible to choose the periods $\Omega_{k}$ a priori in such a way that $\lambda(k)$ extends to a continuous, or even analytic, function in a neighborhood of $2 \in \mathcal{X}$. An understanding of this issue is not germane to the proofs of the theorems in this article.

We note the following non-vanishing property of the scalars $\lambda(k)$ of Theorem 1.5.

Proposition 1.7. There is a neighborhood $U$ of $2 \in \mathcal{X}$ with $\lambda(k) \neq 0$, for all $k \in U \cap \mathbb{Z}^{\geq 2}$.

Proof. The set of $k$ for which $\rho_{k}\left(\mu_{*}\right)=0$ is a finite subset of $\mathbb{Z}$ which does not contain 2 .

By shrinking $U$ if necessary, we may therefore assume that the scalars $\lambda(k)$ are non-zero, for all $k \in U \cap \mathbb{Z}^{\geq 2}$. Note that, even when the periods $\Omega_{k}$ have been chosen, the measure-valued modular symbol $\mu_{*}$ is only defined up to multiplication by an element $\alpha \in \Lambda^{\dagger}$ satisfying $\alpha(2)=1$.

Let $\mathbb{D}$ denote the module of compactly supported measures on

$$
\mathcal{W}:=\mathbb{Q}_{p}^{2}-\{(0,0)\} .
$$


The space $\mathbb{D}_{*}$ is contained in $\mathbb{D}$ by viewing elements of $\mathbb{D}$ as measures with support contained in $L_{*}^{\prime}$. Write $\mathbb{D}^{\dagger}:=\mathbb{D} \otimes \Lambda^{\dagger}$. It is convenient to attach to $\mu_{*}$ a family $\mu_{L}$ of $\mathbb{D}^{\dagger}$-valued modular symbols, indexed by the $\mathbb{Z}_{p}$-lattices $L$ in $\mathbb{Q}_{p}^{2}$. This is done by exploiting the action of the group

$$
\Gamma:=\left\{\gamma=\left(\begin{array}{ll}
a & b \\
c & d
\end{array}\right) \in \mathbf{G L}_{2}(\mathbb{Z}[1 / p]) \text { with } M \mid c, \operatorname{det}(\gamma)>0 .\right\} .
$$

Proposition 1.8. There exists a unique collection $\left\{\mu_{L}\right\}$ of $\mathbb{D}^{\dagger}$-valued modular symbols, indexed by the $\mathbb{Z}_{p}$-lattices $L \subset \mathbb{Q}_{p}^{2}$, and satisfying:

(1) $\mu_{L_{*}}=\mu_{*}$;

(2) For all $\gamma \in \Gamma$, and all compact open $X \subset \mathcal{W}$,

$$
\int_{\gamma X}\left(F \mid \gamma^{-1}\right) d \mu_{\gamma L}\{\gamma r \rightarrow \gamma s\}=\int_{X} F d \mu_{L}\{r \rightarrow s\} .
$$

Proof. The group $\Gamma$ acts transitively on the collection of $\mathbb{Z}_{p}$-lattices in $\mathbb{Q}_{p}^{2}$. The stabiliser of $L_{*}$ in $\Gamma$ for this action is the group $\Gamma_{0}(M)$. Since the modular symbol $\mu_{*}$ is fixed under the action of $\Gamma_{0}(M)$, the rule of (19) is well-defined and determines $\mu_{L}$ uniquely, for each lattice $L$.

For future reference, some of the salient properties of the system $\mu_{L}$ are recorded in a sequence of lemmas.

Lemma 1.9. Let $L$ be a lattice. The distributions $\mu_{L}\{r \rightarrow s\}$ are supported on $L^{\prime}$, for all $r, s \in \mathbb{P}_{1}(\mathbb{Q})$.

Proof. The distributions $\mu_{L_{*}}\{r \rightarrow s\}=\mu_{*}\{r \rightarrow s\}$ are supported on $L_{*}^{\prime}$, for all $r, s \in \mathbb{P}_{1}(\mathbb{Q})$. The lemma follows from the $\Gamma$-equivariance property defining the distributions $\mu_{L}\{r \rightarrow s\}$ at other lattices $L$.

Lemma 1.10. Suppose that $L_{2} \subset L_{1}$ is a sublattice of index $p$ in $L_{1}$. Then for all $k \in U \cap \mathbb{Z}^{\geq 2}$, for all homogeneous functions $F$ on $L_{1}^{\prime} \cap L_{2}^{\prime}$ of degree $k-2$, and for all $r, s \in \mathbb{P}_{1}(\mathbb{Q})$, we have

$$
\int_{L_{1}^{\prime} \cap L_{2}^{\prime}} F d \mu_{L_{2}}\{r \rightarrow s\}=a_{p}(k) \int_{L_{1}^{\prime} \cap L_{2}^{\prime}} F d \mu_{L_{1}}\{r \rightarrow s\} .
$$

Proof. Since the group $\Gamma$ acts transitively on the pairs $\left(L_{1}, L_{2}\right)$ of lattices satisfying $\left[L_{1}: L_{2}\right]=p$, it is enough to prove the lemma in the case where

$$
L_{1}=\frac{1}{p} \mathbb{Z}_{p}+\mathbb{Z}_{p}, \quad L_{2}=\mathbb{Z}_{p}^{2},
$$

so that

$$
L_{1}^{\prime} \cap L_{2}^{\prime}=\mathbb{Z}_{p} \times \mathbb{Z}_{p}^{\times}
$$


For each $a=0, \ldots, p-1$, let $L_{(a)}$ denote the $\mathbb{Z}_{p}$-lattice

$$
L_{(a)}:=\left\{(x, y) \in \mathbb{Z}_{p}^{2} \text { such that } p \mid x+a y\right\} .
$$

The reader will note that

$$
L_{2}^{\prime} \cap L_{(a)}^{\prime}=\left\{(x, y) \in \mathbb{Z}_{p} \times \mathbb{Z}_{p}^{\times} \text {such that } x / y \equiv-a \quad(\bmod p)\right\},
$$

and hence $L_{1}^{\prime} \cap L_{2}^{\prime}$ can be written as a disjoint union

$$
L_{1}^{\prime} \cap L_{2}^{\prime}=\bigcup_{a=0}^{p-1} L_{2}^{\prime} \cap L_{(a)}^{\prime}
$$

In particular,

$$
\int_{L_{1}^{\prime} \cap L_{2}^{\prime}} F d \mu_{L_{2}}\{r \rightarrow s\}=\sum_{a=0}^{p-1} \int_{L_{2}^{\prime} \cap L_{(a)}^{\prime}} F d \mu_{L_{2}}\{r \rightarrow s\} .
$$

Recall the matrices $\gamma_{a}=\left(\begin{array}{ll}1 & a \\ 0 & p\end{array}\right)$ that were introduced earlier in describing the Hecke operator $U_{p}$, and observe that

$$
\gamma_{a} L_{2}=p L_{1}, \quad \gamma_{a} L_{(a)}=p L_{2}, \quad \text { so that } \gamma_{a}\left(L_{2}^{\prime} \cap L_{(a)}^{\prime}\right)=p\left(L_{1}^{\prime} \cap L_{2}^{\prime}\right) .
$$

Using the invariance under $\gamma_{a}$ of the $a$-th term in (20), this expression can be rewritten as:

$$
\begin{aligned}
\int_{L_{1}^{\prime} \cap L_{2}^{\prime}} F d \mu_{L_{2}}\{r \rightarrow s\} & =\sum_{a=0}^{p-1} \int_{p\left(L_{1}^{\prime} \cap L_{2}^{\prime}\right)}\left(F \mid \gamma_{a}^{-1}\right) d \mu_{p L_{1}}\left\{\gamma_{a} r \rightarrow \gamma_{a} s\right\} \\
& =\sum_{a=0}^{p-1} \int_{L_{1}^{\prime} \cap L_{2}^{\prime}}\left(F \mid p \gamma_{a}^{-1}\right) d \mu_{L_{1}}\left\{\gamma_{a} r \rightarrow \gamma_{a} s\right\} \\
& =\int_{L_{1}^{\prime} \cap L_{2}^{\prime}} F d\left(U_{p} \mu_{L_{1}}\right)\{r \rightarrow s\} \\
& =a_{p}(k) \int_{L_{1}^{\prime} \cap L_{2}^{\prime}} F d \mu_{L_{1}}\{r \rightarrow s\} .
\end{aligned}
$$

where the penultimate equality follows from (18). The result follows. 
1.4. The Mazur-Kitagawa $p$-adic $L$-functions. We use the $\mathbb{D}_{*}^{\dagger}$-valued modular symbol $\mu_{*}$ to define the Mazur-Kitagawa two-variable $p$-adic $L$-function attached to $f$ and a Dirichlet character $\chi$, as follows:

Definition 1.11. Let $\chi$ be a primitive quadratic Dirichlet character of conductor $m$ satisfying $\chi(-1)=w_{\infty}$. The Mazur-Kitagawa two-variable $p$-adic $L$-function attached to $\chi$ is the function of $(k, s) \in U \times \mathcal{X}$ defined by the rule

$$
L_{p}\left(f_{\infty}, \chi, k, s\right)=\sum_{a=1}^{m} \chi(a p) \int_{\mathbb{Z}_{p}^{\times} \times \mathbb{Z}_{p}^{\times}}\left(x-\frac{p a}{m} y\right)^{s-1} y^{k-s-1} d \mu_{*}\left\{\infty \rightarrow \frac{p a}{m}\right\} .
$$

The function $L_{p}\left(f_{\infty}, \chi, k, s\right)$ satisfies the following interpolation property with respect to special values of the classical $L$-functions $L\left(f_{k}, \chi, s\right)$.

Theorem 1.12. Suppose that $k$ belongs to $U \cap \mathbb{Z}^{\geq 2}$, and that $1 \leq j \leq k-1$ satisfies $\chi(-1)=(-1)^{j-1} w_{\infty}$. Then

$$
L_{p}\left(f_{\infty}, \chi, k, j\right)=\lambda(k)\left(1-\chi(p) a_{p}(k)^{-1} p^{j-1}\right) L^{*}\left(f_{k}, \chi, j\right) .
$$

Proof. Let $L_{1}$ and $L_{2}$ denote the $\mathbb{Z}_{p}$ lattices that are defined in the proof of Lemma 1.10. The integral that appears in the definition of $L_{p}\left(f_{\infty}, \chi, k, j\right)$ can be expressed as a difference of two contributions

$$
\int_{\mathbb{Z}_{p}^{\times} \times \mathbb{Z}_{p}^{\times}}\left(x-\frac{p a}{m} y\right)^{j-1} y^{k-j-1} d \mu_{*}\left\{\infty \rightarrow \frac{p a}{m} y\right\}=V_{1}-V_{2},
$$

where

$$
\begin{aligned}
V_{1} & =\int_{\mathbb{Z}_{p} \times \mathbb{Z}_{p}^{\times}}\left(x-\frac{p a}{m} y\right)^{j-1} y^{k-j-1} d \mu_{L_{2}}\left\{\infty \rightarrow \frac{p a}{m}\right\} \\
& =\lambda(k) I_{f_{k}}\left\{\infty \rightarrow \frac{p a}{m}\right\}\left(\left(x-\frac{p a}{m} y\right)^{j-1} y^{k-j-1}\right) \\
& =\lambda(k) I_{f_{k}}[j, p a, m],
\end{aligned}
$$

and

$$
\begin{aligned}
V_{2} & =\int_{p \mathbb{Z}_{p} \times \mathbb{Z}_{p}^{\times}}\left(x-\frac{p a}{m} y\right)^{j-1} y^{k-j-1} d \mu_{L_{2}}\left\{\infty \rightarrow \frac{p a}{m}\right\} \\
& =p^{j-1} \int_{\mathbb{Z}_{p} \times \mathbb{Z}_{p}^{\times}}\left(x-\frac{a}{m} y\right)^{j-1} y^{k-j-1} d \mu_{L_{1}}\left\{\infty \rightarrow \frac{a}{m}\right\},
\end{aligned}
$$

where this last equality follows from (19) with $\gamma=\left(\begin{array}{cc}1 / p & 0 \\ 0 & 1\end{array}\right)$. Hence by 
Lemma 1.10,

(28)

$$
\begin{aligned}
V_{2} & =a_{p}(k)^{-1} p^{j-1} \int_{\mathbb{Z}_{p} \times \mathbb{Z}_{p}^{\times}}\left(x-\frac{a}{m} y\right)^{j-1} y^{k-j-1} d \mu_{L_{2}}\left\{\infty \rightarrow \frac{a}{m}\right\} \\
& =\lambda(k) a_{p}(k)^{-1} p^{j-1} I_{f_{k}}\left\{\infty \rightarrow \frac{a}{m}\right\}\left(\left(x-\frac{a}{m} y\right)^{j-1} y^{k-j-1}\right) \\
& =\lambda(k) a_{p}(k)^{-1} p^{j-1} I_{f_{k}}[j, a, m] .
\end{aligned}
$$

Combining (25), (27), and (29) with Definition 1.11 yields

$$
\begin{aligned}
L_{p}\left(f_{\infty}, \chi, k, j\right)= & \lambda(k) \sum_{a=1}^{m} \chi(p a) I_{f_{k}}[j, p a, m] \\
& -\lambda(k) a_{p}(k)^{-1} p^{j-1} \sum_{a=1}^{m} \chi(a p) I_{f_{k}}[j, a, m] \\
= & \lambda(k)\left(1-\chi(p) a_{p}(k)^{-1} p^{j-1}\right) L^{*}\left(f_{k}, \chi, j\right),
\end{aligned}
$$

where the last equation follows from Proposition 1.3.

Remark 1.13. Setting $j=1$ in (26) and (28) and summing over the $a \in$ $\mathbb{Z} / m \mathbb{Z}$ shows that

$$
L_{p}\left(f_{\infty}, \chi, k, 1\right)=\left(1-\chi(p) a_{p}(k)^{-1}\right) L_{p}^{*}\left(f_{\infty}, \chi, k\right),
$$

where

$$
L_{p}^{*}\left(f_{\infty}, \chi, k\right):=\sum_{a=1}^{m} \chi(a) \int_{\mathbb{Z}_{p} \times \mathbb{Z}_{p}^{\times}} y^{k-2} d \mu_{*}\left\{\infty \rightarrow \frac{a}{m}\right\} .
$$

The expression for $L_{p}^{*}\left(f_{\infty}, \chi, k\right)$ extends to a $p$-adic analytic function of $k \in U$, called the improved p-adic L-function in [GS]. The factorisation (30) plays an important role in the proof by Greenberg and Stevens of the exceptional zero conjecture of Mazur, Tate and Teitelbaum.

Theorem 1.12 can also be written in terms of the form $f_{k}^{\sharp}$ of (13), so that $L_{p}\left(f_{\infty}, \chi, k, j\right)$ is equal to

$$
\lambda(k)\left(1-\chi(p) a_{p}(k)^{-1} p^{j-1}\right)\left(1-\chi(p) a_{p}(k)^{-1} p^{k-j-1}\right) L^{*}\left(f_{k}^{\sharp}, \chi, j\right) .
$$

Note in particular that, after specialising at $j=k / 2$, one finds

$$
L_{p}\left(f_{\infty}, \chi, k, k / 2\right)=\lambda(k)\left(1-\chi(p) a_{p}(k)^{-1} p^{\frac{k}{2}-1}\right)^{2} L^{*}\left(f_{k}^{\sharp}, \chi, k / 2\right) .
$$

It will be important for our later study that the Euler factor appearing in this formula is a square. 


\section{Modular forms on quaternion algebras}

2.1. Basic definitions. Let $N$ be a postive integer admitting a factorisation as a product of three relatively prime integers

$$
N=p N^{+} N^{-},
$$

where $p$ is a prime and $N^{-}$is the square-free product of an odd number of primes. Let $B$ be the definite quaternion algebra over $\mathbb{Q}$ ramified exactly at the primes dividing $N^{-}$. That is to say, the algebra $B \otimes \mathbb{R}$ is isomorphic to Hamilton's real quaternions, and for each prime $\ell$ the ring $B_{\ell}:=B \otimes_{\mathbb{Q}} \mathbb{Q}_{\ell}$ is isomorphic to the matrix algebra $M_{2}\left(\mathbb{Q}_{\ell}\right)$ if $\ell$ does not divide $N^{-}$, and to the quaternion division algebra over $\mathbb{Q}_{\ell}$ otherwise. The assumption on $N^{-}$ ensures the existence of this quaternion algebra.

Let $\hat{\mathbb{Z}}$ be the profinite completion of $\mathbb{Z}$, and for any ring $Z$ write

$$
\hat{Z}:=Z \otimes \hat{\mathbb{Z}}
$$

Thus, for example, $\hat{\mathbb{Q}}$ is the ring of finite adèles of $\mathbb{Q}$ and

$$
\hat{B}^{\times}=B^{\times}(\hat{\mathbb{Q}}) \subset \prod_{\ell} B_{\ell}^{\times}
$$

is the group of adèlic points of $B^{\times}$viewed as an algebraic group over $\mathbb{Q}$. Given $b \in \hat{B}^{\times}$, denote by $b_{p} \in B_{p}^{\times}$its component at $p$.

Since $p$ does not divide $N^{-}$, we may fix an isomorphism of $\mathbb{Q}_{p}$-algebras

$$
\iota_{p}: B_{p}=B \otimes_{\mathbb{Q}} \mathbb{Q}_{p} \longrightarrow M_{2}\left(\mathbb{Q}_{p}\right)
$$

and denote by the same letter the corresponding identification of $B_{p}^{\times}$with $\mathbf{G L}_{2}\left(\mathbb{Q}_{p}\right)$. Let $\Sigma=\prod_{\ell} \Sigma_{\ell}$ be a compact open subgroup of $\hat{B}^{\times}$, and let $A$ be any $\mathbb{Q}_{p}$-vector space (or sometimes, $\mathbb{Z}_{p}$-module) equipped with a linear left action of the semigroup $M_{2}\left(\mathbb{Z}_{p}\right)$ of matrices with entries in $\mathbb{Z}_{p}$ and non-zero determinant.

Definition 2.1. An $A$-valued automorphic form on $B^{\times}$of level $\Sigma$ is a function

$$
\phi: \hat{B}^{\times} \longrightarrow A
$$

satisfying

$$
\phi(g b \sigma)=\iota_{p}\left(\sigma_{p}^{-1}\right) \cdot \phi(b),
$$

for all $g \in B^{\times}, b \in \hat{B}^{\times}$, and $\sigma \in \Sigma$.

Denote by $S(\Sigma ; A)$ the space of such forms. 
Remark 2.2. Definition 2.1 differs from the one that is encountered in [BD1]-[BDIS], where an automorphic form on $B^{\times}$"of level $\Sigma$ " is defined to be a function $\tilde{\phi}: \hat{B}^{\times} \longrightarrow A$ satisfying

$$
\tilde{\phi}(g b \sigma)=\iota_{p}(g) \tilde{\phi}(b), \quad \text { for all } g \in B^{\times}, b \in \hat{B}^{\times}, \sigma \in \Sigma .
$$

One passes from one definition to the other by setting

$$
\tilde{\phi}(b)=\iota_{p}\left(b_{p}\right) \phi(b) .
$$

Remark 2.3. An element of $S(\Sigma ; A)$ is completely determined by its values on a set of representatives for the double coset space

$$
\mathbb{X}_{\Sigma}:=B^{\times} \backslash \hat{B}^{\times} / \Sigma,
$$

which is finite (since it is both compact, and discrete). Hence if $A$ is finite dimensional, the same is true for $S(\Sigma ; A)$.

We will primarily be interested in three situations:

(1) The case where $A=\mathbb{Z}_{p}$ with trivial action. In that case the $A$-valued automorphic forms of level $\Sigma$ are said to be of weight 2 and the $\mathbb{Z}_{p}$-module of such forms is denoted $S_{2}(\Sigma)$.

(2) Let $\mathfrak{P}_{k}\left(\mathbb{Q}_{p}\right)$ and $V_{k}\left(\mathbb{Q}_{p}\right)$ denote the spaces introduced in Sect. 1.1 with the left and right actions by $\mathbf{G L}_{2}\left(\mathbb{Q}_{p}\right)$ described in (10) and (11) respectively. The $V_{k}\left(\mathbb{Q}_{p}\right)$-valued automorphic forms of level $\Sigma$ are said to be of weight $k$ and the $\mathbb{Q}_{p}$-vector space of such forms is denoted $S_{k}(\Sigma)$. (The reason for this terminology will become apparent in Sect. 2.3.)

(3) If $\Sigma_{p}=\mathbf{G L}_{2}\left(\mathbb{Z}_{p}\right)$, and $A$ is the module $\mathbb{D}_{*}$ or $\mathbb{D}_{*}^{\dagger}$ defined in Sect. 1.3, an $A$-valued modular form of level $\Sigma$ is referred to as a $p$-adic family of modular forms, and the space of such modular forms is denoted $S_{\infty}(\Sigma)$ or $S_{\infty}^{\dagger}(\Sigma)$, depending on whether $A=\mathbb{D}_{*}$ or $\mathbb{D}_{*}^{\dagger}$. The spaces $S_{\infty}(\Sigma)$ and $S_{\infty}^{\dagger}(\Sigma)$ are equipped with a natural action of $\Lambda$ and $\Lambda^{\dagger}$ respectively, which commute with the Hecke operators. Let $\Sigma^{\prime}$ be the level structure obtained from $\Sigma$ by replacing $\Sigma_{p}=\mathbf{G L}_{2}\left(\mathbb{Z}_{p}\right)$ by the subgroup $\Gamma_{0}\left(p \mathbb{Z}_{p}\right)$ of matrices which are upper triangular modulo $p$. Recall the $\Gamma_{0}\left(p \mathbb{Z}_{p}\right)$-equivariant "weight $k$ specialisation" homomorphism $\rho_{k}: \mathbb{D}_{*} \longrightarrow V_{k}$ defined in (16) of Sect. 1.3. In this chapter, it is convenient to adopt a slightly different definition, by setting

$$
\rho_{k}(\mu)(P):=\int_{\mathbb{Z}_{p}^{\times} \times p \mathbb{Z}_{p}} P(x, y) d \mu(x, y) .
$$

Thus we are now integrating on the region which is complementary to the region $\mathbb{Z}_{p} \times \mathbb{Z}_{p}^{\times}$that appears in (16). Since this region is also preserved by $\Gamma_{0}\left(p \mathbb{Z}_{p}\right)$, the specialisation map $\rho_{k}$ is $\Gamma_{0}\left(p \mathbb{Z}_{p}\right)$-equivari- 
ant and gives rise to homomorphisms, denoted $\rho_{k}$ as well by abuse of notation:

$$
\rho_{k}: S_{\infty}(\Sigma) \longrightarrow S_{k}\left(\Sigma^{\prime}\right) .
$$

As in Sect. 1.3, these specialisation maps allow us to think of $S_{\infty}(\Sigma)$ as a space of families of modular forms of level $\Sigma^{\prime}$ and "varying weights".

The level structures $\Sigma$ that interest us will be of the form $\Sigma=\Sigma_{0}\left(N^{+}, N^{-}\right)$, where $N^{+}$is any integer that is relatively prime to $N^{-}$. To describe this compact open subgroup precisely, choose a maximal order $\mathcal{R}$ in $B$ satisfying

$$
\iota_{p}\left(\mathcal{R} \otimes \mathbb{Z}_{p}\right)=M_{2}\left(\mathbb{Z}_{p}\right) .
$$

(Note that such an order is not unique, even up to conjugation by elements of $B^{\times}$in general, since $B$ is a definite quaternion algebra and therefore does not satisfy the Eichler condition.) For each place $\ell$ of $\mathbb{Q}$ not dividing $N^{-}$, the ring $\mathcal{R} \otimes \mathbb{Z}_{\ell}$ is isomorphic to the $2 \times 2$ matrix ring $M_{2}\left(\mathbb{Z}_{\ell}\right)$. For each $\ell$ not dividing $N^{-}$, fix an isomorphism

$$
\iota_{\ell}: B \otimes \mathbb{Q}_{\ell} \longrightarrow M_{2}\left(\mathbb{Q}_{\ell}\right)
$$

sending $\mathcal{R} \otimes \mathbb{Z}_{\ell}$ to $M_{2}\left(\mathbb{Z}_{\ell}\right)$. The compact open subgroup $\Sigma_{0}\left(N^{+}, N^{-}\right)=$ $\prod_{\ell} \Sigma_{\ell}$ is then defined by letting $\Gamma_{0}\left(N^{+} \mathbb{Z}_{\ell}\right)$ be the subgroup of $\mathbf{G L}_{2}\left(\mathbb{Z}_{\ell}\right)$ consisting of matrices that are upper triangular modulo $N^{+}$, and setting

$$
\Sigma_{\ell}= \begin{cases}\left(\mathcal{R} \otimes \mathbb{Z}_{\ell}\right)^{\times} & \text {if } \ell \mid N^{-}, \\ \iota_{\ell}^{-1}\left(\Gamma_{0}\left(N^{+} \mathbb{Z}_{\ell}\right)\right) & \text { otherwise. }\end{cases}
$$

Denote by

$$
\mathbb{X}_{0}\left(N^{+}, N^{-}\right)=B^{\times} \backslash \hat{B}^{\times} / \Sigma_{0}\left(N^{+}, N^{-}\right)
$$

the corresponding double coset space.

The strong approximation theorem for $B$ ([Vi], p. 61) asserts that, for any compact open subgroup $\Sigma$ of $\hat{B}^{\times}$,

$$
\hat{B}^{\times}=B^{\times} B_{p}^{\times} \Sigma .
$$

Hence the double coset space $\mathbb{X}_{0}\left(p N^{+}, N^{-}\right)$can be rewritten as

$$
\mathbb{X}_{0}\left(p N^{+}, N^{-}\right)=R^{\times} \backslash B_{p}^{\times} / \iota_{p}^{-1}\left(\Gamma_{0}\left(p \mathbb{Z}_{p}\right)\right)=\tilde{\Gamma} \backslash \mathbf{G L}_{2}\left(\mathbb{Q}_{p}\right) / \Gamma_{0}\left(p \mathbb{Z}_{p}\right),
$$

where

$$
\begin{aligned}
& R:=\left\{x \in \mathcal{R}[1 / p] \text { such that } \iota_{\ell}(x) \in \mathbb{Z}_{\ell}\left[\Gamma_{0}\left(N \mathbb{Z}_{\ell}\right)\right], \text { for all } \ell \mid N^{+}\right\}, \\
& \tilde{\Gamma}:=\iota_{p}\left(R^{\times}\right) .
\end{aligned}
$$

The ring $R$ is a so-called "Eichler $\mathbb{Z}[1 / p]$-order of level $N^{+}$" in $B$. Such $\mathbb{Z}[1 / p]$-orders are unique, up to conjugation by elements of $B^{\times}$. Thanks 
to (35), a modular form in $S_{k}\left(\Sigma_{0}\left(p N^{+}, N^{-}\right)\right)$can be viewed as a function $\phi: \mathbf{G L}_{2}\left(\mathbb{Q}_{p}\right) \longrightarrow V_{k}$ satisfying

$$
\phi(\gamma b \sigma)=\sigma^{-1} \phi(b), \quad \text { for all } \gamma \in \tilde{\Gamma}, b \in \mathbf{G L}_{2}\left(\mathbb{Q}_{p}\right) \text {, and } \sigma \in \Gamma_{0}\left(p \mathbb{Z}_{p}\right) \text {. }
$$

It is this point of view that will be taken from now on.

The spaces $S\left(\Sigma_{0}\left(p N^{+} ; N^{-}\right) ; A\right)$ are equipped with an action of Hecke operators $T_{\ell}$ indexed by the rational primes which do not divide $N$, defined as in [Gr] for example. If $\ell$ is a prime that does not divide $p N^{+} N^{-}$, then the Hecke operator $T_{\ell}$ on $S_{k}\left(N^{+}, N^{-}\right)$is defined in terms of the double coset decomposition

$$
\mathbf{G L}_{2}\left(\mathbb{Z}_{\ell}\right)\left(\begin{array}{ll}
\ell & 0 \\
0 & 1
\end{array}\right) \mathbf{G L}_{2}\left(\mathbb{Z}_{\ell}\right)=\bigcup_{a=0}^{\ell} \sigma_{a}(\ell) \mathbf{G} \mathbf{L}_{2}\left(\mathbb{Z}_{\ell}\right)
$$

by the rule

$$
\left(T_{\ell} \phi\right)(b)(P)=\sum_{a=0}^{\ell} \phi\left(b \sigma_{a}(\ell)\right)\left(P \mid \sigma_{a}(\ell)\right) .
$$

The Hecke operator $U_{p}$ plays a particularly important role in our discussion. It is defined in terms of the decomposition

$$
\Gamma_{0}\left(p \mathbb{Z}_{p}\right)\left(\begin{array}{ll}
1 & 0 \\
0 & p
\end{array}\right) \Gamma_{0}\left(p \mathbb{Z}_{p}\right)=\bigcup_{a=0}^{p-1} \sigma_{a} \Gamma_{0}\left(p \mathbb{Z}_{p}\right)
$$

of the double coset attached to the matrix $\operatorname{diag}(1, p) \in \mathbf{G L}_{2}\left(\mathbb{Q}_{p}\right)$ as a disjoint union of left cosets. Since $\Gamma_{0}\left(p \mathbb{Z}_{p}\right)$ is the stabiliser in $\mathbf{G L}_{2}\left(\mathbb{Q}_{p}\right)$ of the lattices $\mathbb{Z}_{p}^{2}$ and $\mathbb{Z}_{p} \oplus p \mathbb{Z}_{p}$, the double coset appearing in (38) consists of matrices in $\mathbf{G L}_{2}\left(\mathbb{Q}_{p}\right)$ which send the lattice $\mathbb{Z}_{p}^{2}$ to $\mathbb{Z}_{p} \oplus p \mathbb{Z}_{p}$. Let $L(0), L(1)$, $\ldots, L(p-1)$ denote the $p$ sublattices of index $p$ of $\mathbb{Z}_{p} \oplus p \mathbb{Z}_{p}$ which are different from $p\left(\mathbb{Z}_{p}^{2}\right)$. The matrices $\sigma_{a}$ appearing in (38) can be arranged so that

$$
\sigma_{a}\left(\mathbb{Z}_{p}^{2}\right)=\mathbb{Z}_{p} \oplus p \mathbb{Z}_{p}, \quad \sigma_{a}\left(\mathbb{Z}_{p} \oplus p \mathbb{Z}_{p}\right)=L(a)
$$

Note, for example, that (39) determines $\sigma_{a}$ uniquely up to multiplication by elements of $\Gamma_{0}\left(p \mathbb{Z}_{p}\right)$ on the right. The Hecke operator $U_{p}$ acting on $S\left(\Sigma_{0}\left(p N^{+}, N^{-}\right) ; A\right)$ is defined by

$$
\left(U_{p} \phi\right)(b)=\sum_{a=0}^{p-1} \sigma_{a} \phi\left(b \sigma_{a}\right) .
$$


In particular, if $\phi$ belongs to $S_{k}\left(\Sigma_{0}\left(p N^{+}, N^{-}\right)\right)$, then

$$
\left(U_{p} \phi\right)(b)(P)=\sum_{a=0}^{p-1} \phi\left(b \sigma_{a}\right)\left(P \mid \sigma_{a}\right),
$$

for any $b \in \mathbf{G L}_{2}\left(\mathbb{Q}_{p}\right)$, and all $P \in \mathfrak{P}_{k}$, while if $\phi$ belongs to the space $S_{\infty}\left(\Sigma_{0}\left(N^{+}, N^{-}\right)\right)^{\dagger}$, and has $U$ as a neighborhood of regularity, then

$$
\int_{X} F d\left(U_{p} \phi\right)(b)=\sum_{a=0}^{p-1} \int_{\sigma_{a}^{-1} X}\left(F \mid \sigma_{a}\right) d \phi\left(b \sigma_{a}\right),
$$

for all compact open $X \subset L_{*}^{\prime}$ and all homogeneous $F$ of degree $k-2$ with $k \in U$.

For future reference we note the following relation

$$
\mathbb{Z}_{p}^{\times} \times p \mathbb{Z}_{p}=\bigcup_{a=0}^{p-1} \sigma_{a}\left(\mathbb{Z}_{p}^{\times} \times p \mathbb{Z}_{p}\right) .
$$

This relation can be rewritten as

$$
\left(\mathbb{Z}_{p}^{2}\right)^{\prime} \cap\left(\mathbb{Z}_{p} \oplus p \mathbb{Z}_{p}\right)^{\prime}=\bigcup_{a=0}^{p-1}\left(\mathbb{Z}_{p} \oplus p \mathbb{Z}_{p}\right)^{\prime} \cap L(a)^{\prime},
$$

which can be checked by specialising to any specific collection of lattices satisfying the same inclusion relations as $\mathbb{Z}_{p}^{2}, \mathbb{Z}_{p} \oplus p \mathbb{Z}_{p}, L(0), \ldots, L(p-1)$, using the fact that $\mathbf{G L}_{2}\left(\mathbb{Q}_{p}\right)$ acts transitively on such collections.

2.2. The Bruhat-Tits tree. A modular form $\eta \in S\left(\Sigma_{0}\left(N^{+}, N^{-}\right) ; A\right)$ gives rise to a $\tilde{\Gamma}$-equivariant $A$-valued function on the set of $\mathbb{Z}_{p}$-lattices in $\mathbb{Q}_{p}^{2}$, by setting

$$
c_{\eta}(L)=g_{L} \eta\left(g_{L}\right),
$$

where $g_{L}$ is any element of $\mathbf{G L}_{2}\left(\mathbb{Q}_{p}\right)$ satisfying $g_{L}\left(\mathbb{Z}_{p}^{2}\right)=L$. Likewise, if $\eta$ belongs to $S\left(\Sigma_{0}\left(p N^{+}, N^{-}\right) ; A\right)$, it gives rise to a $\tilde{\Gamma}$-equivariant $A$-valued function on the set of pairs $\left(L_{1}, L_{2}\right)$ of $\mathbb{Z}_{p}$-lattices in $\mathbb{Q}_{p}^{2}$ for which $L_{1}$ contains $L_{2}$ with index $p$, by setting

$$
c_{\eta}\left(L_{1}, L_{2}\right)=g_{L_{1}, L_{2}} \eta\left(g_{L_{1}, L_{2}}\right),
$$

where $g_{L_{1}, L_{2}}$ is any element of $\mathbf{G L}_{2}\left(\mathbb{Q}_{p}\right)$ satisfying

$$
g_{L_{1}, L_{2}}\left(\mathbb{Z}_{p}^{2}\right)=L_{1}, \quad g_{L_{1}, L_{2}}\left(\mathbb{Z}_{p} \oplus p \mathbb{Z}_{p}\right)=L_{2} .
$$


The Hecke operators $T_{p}$ and $U_{p}$ acting on $S\left(\Sigma_{0}\left(N^{+}, N^{-}\right) ; A\right)$ and on $S\left(\Sigma_{0}\left(p N^{+}, N^{-}\right) ; A\right)$ respectively admit a particularly simple expression in terms of the associated function on lattices, namely:

$$
\begin{aligned}
c_{T_{p} \eta}(L) & =\sum_{\tilde{L} \subset L} c_{\eta}(\tilde{L}), \\
c_{U_{p} \eta}\left(L_{1}, L_{2}\right) & =\sum_{\tilde{L} \subset L_{2}} c_{\eta}\left(L_{2}, \tilde{L}\right),
\end{aligned}
$$

where the first sum is taken over the $p+1$ sublattices of $L$ of index $p$, and the second sum is taken over the $p$ sublattices of index $p$ of $L_{2}$ which are different from $p L_{1}$. These formulae are direct consequences of the definitions; for example, (48) follows from (40) and (45).

There are two natural "degeneracy maps"

$$
d_{1}, d_{2}: S\left(\Sigma_{0}\left(N^{+}, N^{-}\right) ; A\right) \longrightarrow S\left(\Sigma_{0}\left(p N^{+}, N^{-}\right) ; A\right)
$$

which are described as follows in terms of the associated functions on lattices:

$$
c_{d_{1}(\eta)}\left(L_{1}, L_{2}\right):=c_{\eta}\left(L_{1}\right), \quad c_{d_{2}(\eta)}\left(L_{1}, L_{2}\right):=c_{\eta}\left(L_{2}\right) .
$$

These degeneracy maps are compatible with the actions of the Hecke operators $T_{n}$ with $p \not \backslash n$, but do not intertwine the actions of the operators $T_{p}$ and $U_{p}$ on each side.

An eigenvector $\phi^{\sharp} \in S_{k}\left(\Sigma_{0}\left(N^{+}, N^{-}\right)\right)$for the Hecke operator $T_{p}$ is said to be ordinary if the associated eigenvalue $a_{p}\left(\phi^{\sharp}\right)$ is a $p$-adic unit. In that case we can define a $p$-adic unit $a_{p}$ by the rule

$$
x^{2}-a_{p}\left(\phi^{\sharp}\right) x+p^{k-1}=\left(x-a_{p}\right)\left(x-a_{p}^{-1} p^{k-1}\right) .
$$

The form $\phi \in S_{k}\left(\Sigma_{0}\left(p N^{+}, N^{-}\right)\right)$defined by

$$
c_{\phi}\left(L_{1}, L_{2}\right)=c_{\phi^{\sharp}}\left(L_{2}\right)-a_{p}^{-1} c_{\phi^{\sharp}}\left(p L_{1}\right)
$$

is an eigenvector for $U_{p}$ satisfying $U_{p} \phi=a_{p} \phi$. This can be checked by a direct calculation using (47) and (48) and the fact that $c_{\phi}$ and $c_{\phi^{\sharp}}$ are homogenous of degree $k-2$ in the sense that

$$
c_{\phi^{\sharp}}(p L)=p^{k-2} c_{\phi^{\sharp}}(L), \quad \text { and } \quad c_{\phi}\left(p L_{1}, p L_{2}\right)=p^{k-2} c_{\phi}\left(L_{1}, L_{2}\right) .
$$

A $U_{p^{-}}$-eigenform $\phi$ of level $\Sigma_{0}\left(p N^{+}, N^{-}\right)$which is obtained from an eigenform $\phi^{\sharp}$ of level $\Sigma_{0}\left(N^{+}, N^{-}\right)$as in (49) is said to be old at $p$.

When $k=2$ the functions $c_{\phi}$ and $c_{\phi^{\sharp}}$ are defined on homothety classes of lattices. It is then convenient to view $c_{\phi^{\sharp}}$ and $c_{\phi}$ as functions on the vertices and ordered edges respectively of the Bruhat-Tits tree of $\mathbf{G L}_{2}\left(\mathbb{Q}_{p}\right)$. This tree is the graph, denoted $\mathcal{T}$, whose set $\mathcal{T}_{0}$ of vertices is in bijection with the homothety classes of $\mathbb{Z}_{p}$-lattices in $\mathbb{Q}_{p}^{2}$, two such vertices being 
joined by an unordered edge if the corresponding homothety classes admit representatives which are contained in each other with index $p$. Write $\mathcal{T}_{1}$ for the set of unordered edges of $\mathcal{T}$.

The group $\mathbf{G L}_{2}\left(\mathbb{Q}_{p}\right)$, which acts naturally on $\mathbb{Q}_{p}^{2}$ via left multiplication on column vectors, also acts on $\mathcal{T}$, and this action preserves the adjacency relations between vertices of $\mathcal{T}$.

A vertex in $\mathcal{T}_{0}$ is said to be even if its distance from the standard vertex $v_{*}:=\left[\mathbb{Z}_{p}^{2}\right]$ is even, and is said to be odd otherwise. Denoting by $\mathcal{E}(\mathcal{T})$ the set of ordered edges of $\mathcal{T}$ (i.e., the set of ordered pairs of adjacent vertices in $\mathcal{T}$ ), we say that such an edge is even if its origin is even, and is odd otherwise. Thus we have decompositions

$$
\mathcal{T}_{0}=\mathcal{T}_{0}^{+} \cup \mathcal{T}_{0}^{-}, \quad \mathcal{E}(\mathcal{T})=\mathscr{E}(\mathcal{T})^{+} \cup \mathscr{E}(\mathcal{T})^{-}
$$

of the sets $\mathcal{T}_{0}$ and $\mathcal{E}(\mathcal{T})$ respectively into a disjoint union of the subsets of even and odd elements. The group $\mathbf{G L}_{2}\left(\mathbb{Q}_{p}\right)$ acts transitively on $\mathcal{T}_{0}$ and on $\mathcal{T}_{1}$, while the group $\mathbf{S L}_{2}\left(\mathbb{Q}_{p}\right)$ preserves the subsets $\mathcal{T}_{0}^{+}$and $\mathscr{E}(\mathcal{T})^{+}$.

The Petersson scalar product on $S_{k}\left(\Sigma_{0}\left(N^{+}, N^{-}\right)\right)$. Let $U:=\operatorname{ad}^{0}(B)$ be the representation of $B^{\times}$consisting of the elements of $B$ of reduced trace zero, equipped with the right action of $B^{\times}$given by

$$
u \cdot b:=b^{-1} u b .
$$

There is a $\mathbb{Q}$-valued perfect symmetric pairing

$$
\langle,\rangle: U \times U \longrightarrow \mathbb{Q},
$$

defined by $\langle u, v\rangle:=(1 / 2) \operatorname{tr}(u \bar{v})$, where $\operatorname{tr}$ denotes the reduced trace on $B$ and $\bar{v}$ is the image of $v$ by the canonical involution on $B$. This pairing is $B^{\times}$-invariant, in the sense that

$$
\langle u \cdot b, v \cdot b\rangle=\langle u, v\rangle
$$

for all $b \in B^{\times}$. The map sending $u \in U$ to

$$
P_{u}(x, y):=\operatorname{Trace}\left(\iota_{p}(u)\left(\begin{array}{ll}
-x y & x^{2} \\
-y^{2} & x y
\end{array}\right)\right) \in \mathfrak{P}_{4}
$$

defines a surjective homomorphism from $U \otimes \mathbb{C}_{p}$ to $\mathfrak{P}_{4}$, which intertwines the right actions of $B_{1}^{\times}$, the group of elements of $B^{\times}$of reduced norm one, and $\mathbf{S L}_{2}\left(\mathbb{Q}_{p}\right)$ on the first and second space, respectively. This yields a surjection from $\operatorname{Sym}^{k / 2-1}(U) \otimes \mathbb{C}_{p}$ to $\mathfrak{P}_{k}$. The pairing $\langle$,$\rangle induces a perfect$ symmetric pairing $\langle,\rangle_{k}$ on $\operatorname{Sym}^{k / 2-1}(U)$ by setting

$$
\begin{aligned}
\left\langle\left(u_{1}, \ldots, u_{k / 2-1}\right),\left(v_{1}, \ldots, v_{k / 2-1}\right)\right\rangle_{k} & :=\sum_{\sigma \in S_{k / 2-1}}\left\langle u_{1}, v_{\sigma(1)}\right\rangle \cdots\left\langle u_{k / 2-1}, v_{\sigma(k / 2-1)}\right\rangle,
\end{aligned}
$$

$S_{k / 2-1}$ being the symmetric group on $k / 2-1$ letters. 
By dualizing this map, and using $\langle,\rangle_{k}$ to identify $\operatorname{Sym}^{k / 2-1}(U) \otimes \mathbb{C}_{p}$ with its $\mathbb{C}_{p}$-dual, we obtain an inclusion of $V_{k}$ into $\operatorname{Sym}^{k / 2-1}(U) \otimes \mathbb{C}_{p}$. By an abuse of notation, we denote by $\langle,\rangle_{k}$ also the perfect symmetric pairing on $V_{k}$ obtained by restriction.

Given $\eta \in S_{k}\left(\Sigma_{0}\left(N^{+}, N^{-}\right)\right)$, let $c_{\eta}$ be the $\tilde{\Gamma}$-equivariant function on lattices defined earlier in this section. Write $|L|$ for the generalised index of the lattice $L$ in $\mathbb{Z}_{p}^{2}$, defined as the power of $p$ which exactly divides the determinant of any element of $\mathbf{G L}_{2}\left(\mathbb{Q}_{p}\right)$ sending $\mathbb{Z}_{p}^{2}$ to $L$. Since $c_{\eta}$ is homogenous of degree $k-2$, the quantity $|L|^{1-k / 2} c_{\eta}(L)$ depends only on the homothety class $v \in \mathcal{T}_{0}$ of the lattice $L$, and will be denoted $\bar{c}_{\eta}(v)$. Define the Petersson norm of $\eta$ to be

$$
\langle\eta, \eta\rangle:=\sum_{v \in \Gamma \backslash \mathcal{T}_{0}} w_{v} \cdot\left\langle\bar{c}_{\eta}(v), \bar{c}_{\eta}(v)\right\rangle_{k},
$$

where $w_{v}$ denotes the order of the stabiliser of $v$ in $\Gamma$. Note that $\langle\eta, \eta\rangle$ does not depend on the choice of representatives for the finite set $\Gamma \backslash \mathcal{T}_{0}$.

For $\phi \in S_{k}\left(\Sigma_{0}\left(N^{+} p, N^{-}\right)\right.$, it is possible to define its Petersson norm $\langle\eta, \eta\rangle$ in a similar way, with the set $\Gamma \backslash \mathcal{E}(\mathcal{T})$ replacing the role of $\Gamma \backslash \mathcal{T}_{0}$. In particular, given $\phi \in S_{2}\left(\Sigma_{0}\left(N^{+} p, N^{-}\right)\right.$, one has

$$
\langle\phi, \phi\rangle:=\sum_{e \in \Gamma \backslash \mathcal{E}(\mathcal{T})} w_{e} \cdot\left\langle c_{\phi}(e), c_{\phi}(e)\right\rangle_{2},
$$

where $w_{e}$ denotes the order of the stabiliser of $e$ in $\Gamma$.

2.3. The Jacquet-Langlands correspondence. Recall the Hecke congruence group $\Gamma_{0}(N)$ of $\mathbf{S L}_{2}(\mathbb{Z})$, and let $S_{k}\left(\Gamma_{0}(N)\right)$ denote the vector space of classical cusp forms of weight $k$ for $\Gamma_{0}(N)$ with rational Fourier expansion, tensored with $\mathbb{Q}_{p}$. Recall the factorisation $N=p M=p N^{+} N^{-}$of $N$ that was introduced earlier. A modular form in $S_{k}\left(\Gamma_{0}(N)\right)$ is said to be old at $N^{-}$if it can be expressed as a linear combination of modular forms of the form $g(d z)$ where $g(z)$ is of level not divisible by $N^{-}$. The orthogonal complement of this space of $N^{-}$-old forms is called the space of $N^{-}$-new forms on $\Gamma_{0}(N)$, and is denoted by $S_{k}^{\text {new }-N^{-}}\left(\Gamma_{0}(N)\right)$.

The following theorem, a special case of the Jacquet-Langlands correspondence, explains the interest of modular forms on definite quaternion algebras in our study.

Theorem 2.4. There exist Hecke-equivariant isomorphisms

$$
\begin{aligned}
S_{k}\left(\Sigma_{0}\left(N^{+}, N^{-}\right)\right) & \longrightarrow S_{k}^{\text {new }-N^{-}}\left(\Gamma_{0}(M)\right) \\
S_{k}\left(\Sigma_{0}\left(p N^{+}, N^{-}\right)\right) & \longrightarrow S_{k}^{\text {new }-N^{-}}\left(\Gamma_{0}(N)\right) .
\end{aligned}
$$

The assumption that the elliptic curve $E$ of the Introduction has at least one prime $\neq p$ of multiplicative reduction allows us to write its conductor 
as $N=p N^{+} N^{-}$, whose factors satisfy the hypotheses of (32). Hence, the normalised eigenform $f_{k}^{\sharp} \in S_{k}^{\text {new }-N^{-}}\left(\Gamma_{0}(M)\right)$ of weight $k \in U \cap \mathbb{Z}^{>2}$ associated to $f$ via Hida's theory in (12) and (13) corresponds to a modular form $\phi_{k}^{\sharp} \in S_{k}\left(\Sigma_{0}\left(N^{+}, N^{-}\right)\right)$which is an eigenvector for the Hecke operators with the same associated eigenvalues. The form $\phi_{k}^{\sharp}$ is only determined up to multiplication by a non-zero scalar. We normalise it in such a way that

$$
\left\langle\phi_{k}^{\sharp}, \phi_{k}^{\sharp}\right\rangle=1,
$$

so that $\phi_{k}^{\sharp}$ is now determined up to sign. Let $\phi_{k} \in S_{k}\left(\Sigma_{0}\left(p N^{+}, N^{-}\right)\right)$be the eigenvector for $U_{p}$ which is obtained from $\phi_{k}^{\sharp}$ via (49), and let $c_{k}$ and $c_{k}^{\sharp}$ denote the corresponding functions on lattices.

When $k=2$, let $\phi_{2} \in S_{2}\left(\Sigma_{0}\left(p N^{+}, N^{-}\right)\right)$be a modular form associated to $f$ via Theorem 2.4. Since $\phi_{2}$ is new at $p$, we set $\phi_{2}^{\sharp}:=0$. We do not impose a normalising condition on $\phi_{2}$, but assume instead that it takes its values in $\mathbb{Z}$. Note then that the quantity $\left\langle\phi_{2}, \phi_{2}\right\rangle$ is well-defined up to multiplication by elements of $\left(\mathbb{Q}^{\times}\right)^{2}$. Occasionally we will write $\phi$ instead of $\phi_{2}$.

The following result plays the role of Theorem 1.5 in the context of automorphic forms on definite quaternion algebras.

Theorem 2.5. There exists a family $\phi_{\infty} \in S_{\infty}^{\dagger}\left(\Sigma_{0}\left(N^{+}, N^{-}\right)\right)$such that

(1) $\rho_{2}\left(\phi_{\infty}\right)=\phi_{2}$;

(2) Let $U$ be a neighborhood of regularity for the measures $\phi_{\infty}(g) \in \mathbb{D}_{*}^{\dagger}$. For all $k \in U \cap \mathbb{Z}^{\geq 2}$, there exists a scalar $\lambda_{B}(k) \in \mathbb{C}_{p}$ such that

$$
\rho_{k}\left(\phi_{\infty}\right)=\lambda_{B}(k) \phi_{k} .
$$

In other words, for all $P \in \mathfrak{P}_{k}$ and for all $b \in \mathbf{G L}_{2}\left(\mathbb{Q}_{p}\right)$,

$$
\int_{\mathbb{Z}_{p}^{\times} \times p \mathbb{Z}_{p}} P(x, y) d \phi_{\infty}(b)=\lambda_{B}(k) \phi_{k}(b)(P) .
$$

Proof. The proof of Theorem (5.13) of [GS] adapts to the present context mutatis mutandis, after replacing "modular symbols" by "functions on $\hat{B}^{\times}$". See Sect. 6 of [GS] for more details. Further details on the case of definite quaternion algebras are given in some (as yet unpublished) notes of Glenn Stevens.

Remark 2.6. Theorem 2.5 is a consequence of the more general "JacquetLanglands correspondence for families" established in [Ch] which applies also to non-ordinary eigenforms. The methods developed in Sect. 6 of [GS] is sufficient to handle the case of interest in this paper, which is only concerned with Hida families of ordinary eigenforms, and this allows us to work with measures (elements of $\mathbb{D}^{\dagger}$ ) rather than the locally analytic functions and distributions that appear in $[\mathrm{Bu}]$ and $[\mathrm{Ch}]$. 
2.4. Measure-valued forms. Recall the space $\mathbb{D}$ of compactly supported measures on $\mathbb{W}$, which contains $\mathbb{D}_{*}$ in a natural way and is also equipped with a natural left action of the group $\mathbf{G L}_{2}\left(\mathbb{Q}_{p}\right)$ extending the action of $M_{2}\left(\mathbb{Z}_{p}\right)$ on $\mathbb{D}_{*}$.

Guided by the constructions of Sect. 1.3, (cf. in particular Proposition 1.8), we associate to the form $\phi_{\infty}$ of the previous section a collection of measures $\mu_{L} \in \mathbb{D}^{\dagger}$ indexed by the $\mathbb{Z}_{p}$-lattices in $\mathbb{Q}_{p}^{2}$, by setting

$$
\mu_{L}:=g_{L} \phi_{\infty}\left(g_{L}\right)
$$

where $g_{L} \in \mathbf{G L}_{2}\left(\mathbb{Q}_{p}\right)$ is any matrix satisfying $g_{L}\left(\mathbb{Z}_{p}^{2}\right)=L$. The measures $\mu_{L}$ satisfy the basic property

$$
\int_{X} F d \mu_{L}=\int_{g_{L}^{-1} X}\left(F \mid g_{L}\right) d \phi_{\infty}\left(g_{L}\right),
$$

for all compact open $X \subset \mathcal{W}$ and for all homogeneous $F$ of degree $k$ with $k \in U \cap \mathbb{Z}^{\geq 2}$.

We note the following properties of the $\mu_{L}$ :

Lemma 2.7. The measures $\mu_{L}$ are supported on $L^{\prime}$, for all $L$.

Proof. Property (56) implies that $\mu_{L}$ is supported on $g_{L}\left(\left(\mathbb{Z}_{p}^{2}\right)^{\prime}\right)=L^{\prime}$ since the measures $\phi_{\infty}(g)$ are supported on $\left(\mathbb{Z}_{p}^{2}\right)^{\prime}$.

Lemma 2.8. For all $\gamma \in \tilde{\Gamma}$, and all homogeneous functions $F(x, y)$ of degree $k-2$ with $k \in U$,

$$
\int_{\gamma X}\left(F \mid \gamma^{-1}\right) d \mu_{\gamma L}=\int_{X} F d \mu_{L} .
$$

In particular, if $P$ is a homogeneous polynomial of degree $k-2$ with $k \in U \cap \mathbb{Z}^{\geq 2}$,

$$
\int_{p L^{\prime}} P d \mu_{p L}=p^{k-2} \int_{L^{\prime}} P d \mu_{L} .
$$

Proof. This follows from the invariance property of $\phi_{\infty}$ under left multiplication by $\tilde{\Gamma}$.

The following is an analogue of Lemma 1.10 of Sect. 1.3.

Lemma 2.9. Suppose that $L_{2} \subset L_{1}$ is a sublattice of index $p$ in $L_{1}$. Then for all $k \in U$, and for all homogenous functions $F(x, y)$ of degree $k-2$, we have

$$
\int_{L_{1}^{\prime} \cap L_{2}^{\prime}} F d \mu_{L_{2}}=a_{p}(k) \int_{L_{1}^{\prime} \cap L_{2}^{\prime}} F d \mu_{L_{1}} .
$$


Proof. Let $\mathbb{D}^{\prime}$ be the $\Lambda$-module of measures on $\mathbb{Z}_{p}^{\times} \times p \mathbb{Z}_{p}$, and, as a natural extension of the notations adopted previously, write $\left(\mathbb{D}^{\prime}\right)^{\dagger}=\mathbb{D}^{\prime} \otimes \Lambda^{\dagger}$. The restriction map from $\mathbb{D}_{*}$ to $\mathbb{D}^{\prime}$ is $\Gamma_{0}\left(p \mathbb{Z}_{p}\right)$-equivariant and induces an isomorphism

$$
S_{\infty}\left(\Sigma_{0}\left(N^{+}, N^{-}\right)\right) \longrightarrow S\left(\Sigma_{0}\left(p N^{+}, N^{-}\right) ; \mathbb{D}^{\prime}\right) .
$$

The same holds, of course, for the corresponding modules tensored with $\Lambda^{\dagger}$. Let

$$
\phi_{\infty}^{\prime} \in S\left(\Sigma_{0}\left(p N^{+}, N^{-}\right) ; \mathbb{D}^{\prime}\right) \otimes \Lambda^{\dagger}
$$

be the image of $\phi_{\infty}$ under this isomorphism. Note that the matrices $\sigma_{a}$ that appear in formula (40) defining the action of $U_{p}$ satisfy

$$
\sigma_{a}^{-1}\left(\mathbb{Z}_{p}^{\times} \times p \mathbb{Z}_{p}\right) \supset \mathbb{Z}_{p}^{\times} \times p \mathbb{Z}_{p} .
$$

By formula (42) and the fact that $\phi_{\infty}^{\prime}$ is an eigenvector for $U_{p}$ with eigenvalue $a_{p}(k)$, we have

$$
\sum_{a=0}^{p-1} \int_{\mathbb{Z}_{p}^{\times} \times p \mathbb{Z}_{p}}\left(F \mid \sigma_{a}\right) d \phi_{\infty}^{\prime}\left(b \sigma_{a}\right)=a_{p}(k) \int_{\mathbb{Z}_{p}^{\times} \times p \mathbb{Z}_{p}} F d \phi_{\infty}^{\prime}(b),
$$

for all $b \in \mathbf{G L}_{2}\left(\mathbb{Q}_{p}\right)$, for all $k \in U$ and all homogenous $F$ of degree $k-2$. The same relation holds with $\phi_{\infty}^{\prime}$ replaced by $\phi_{\infty}$. By the definition of the action of $\mathbf{G L}_{2}\left(\mathbb{Q}_{p}\right)$ on $\mathbb{D}$, this equation can be rewritten as

$$
\begin{aligned}
& \sum_{a=0}^{p-1} \int_{b \sigma_{a}\left(\mathbb{Z}_{p}^{\times} \times p \mathbb{Z}_{p}\right)}\left(F \mid b^{-1}\right) d\left(b \sigma_{a} \phi_{\infty}\left(b \sigma_{a}\right)\right) \\
&=a_{p}(k) \int_{b\left(\mathbb{Z}_{p}^{\times} \times p \mathbb{Z}_{p}\right)}\left(F \mid b^{-1}\right) d\left(b \phi_{\infty}(b)\right) .
\end{aligned}
$$

Now let $b$ be any matrix in $\mathbf{G L}_{2}\left(\mathbb{Q}_{p}\right)$ such that $b\left(\mathbb{Z}_{p}^{2}\right)=L_{1}$ and $b\left(\mathbb{Z}_{p} \oplus p \mathbb{Z}_{p}\right)$ $=L_{2}$, so that $b\left(\mathbb{Z}_{p}^{\times} \times p \mathbb{Z}_{p}\right)=L_{1}^{\prime} \cap L_{2}^{\prime}$. Then the previous displayed equation becomes:

$$
\sum_{a=0}^{p-1} \int_{b \sigma_{a}\left(\mathbb{Z}_{p}^{\times} \times p \mathbb{Z}_{p}\right)}\left(F \mid b^{-1}\right) d \mu_{L_{2}}=a_{p}(k) \int_{L_{1}^{\prime} \cap L_{2}^{\prime}}\left(F \mid b^{-1}\right) d \mu_{L_{1}} .
$$

Multiplying (43) by $b$ on the left gives the following expression for $L_{1}^{\prime} \cap L_{2}^{\prime}$ as a disjoint union:

$$
L_{1}^{\prime} \cap L_{2}^{\prime}=\bigcup_{a=0}^{p-1} b \sigma_{a}\left(\mathbb{Z}_{p}^{\times} \times p \mathbb{Z}_{p}\right)
$$


Hence it follows that

$$
\int_{L_{1}^{\prime} \cap L_{2}^{\prime}}\left(F \mid b^{-1}\right) d \mu_{L_{2}}=a_{p}(k) \int_{L_{1}^{\prime} \cap L_{2}^{\prime}}\left(F \mid b^{-1}\right) d \mu_{L_{1}} .
$$

Since this is true for any homogenous $F$ of degree $k-2$, the result follows.

Lemma 2.10. For any $L_{1} \supset L_{2}$ with index $p$, and for any polynomial $P$ of degree $k-2$ with $k \in U \cap \mathbb{Z}^{\geq 2}$,

$$
\int_{L_{1}^{\prime} \cap L_{2}^{\prime}} P d \mu_{L_{1}}=\lambda_{B}(k) c_{k}\left(L_{1}, L_{2}\right)(P) .
$$

Proof. The defining property of $\phi_{\infty}$ states that

$$
\int_{\mathbb{Z}_{p}^{\times} \times p \mathbb{Z}_{p}} P d \phi_{\infty}(g)=\lambda_{B}(k) \phi_{k}(g)(P) .
$$

This implies that

$$
\int_{g\left(\mathbb{Z}_{p}^{\times} \times p \mathbb{Z}_{p}\right)}\left(P \mid g^{-1}\right) d\left(g \phi_{\infty}(g)\right)=\lambda_{B}(k) g \phi_{k}(g)\left(P \mid g^{-1}\right) .
$$

Taking $g$ to be the element $g_{L_{1}, L_{2}}$ of (46), this gives

$$
\int_{L_{1}^{\prime} \cap L_{2}^{\prime}}\left(P \mid g^{-1}\right) d \mu_{L_{1}}=\lambda_{B}(k) c_{k}\left(L_{1}, L_{2}\right)\left(P \mid g^{-1}\right) .
$$

The result follows.

Proposition 2.11. For any lattice $L$, for any $k \in U \cap \mathbb{Z}^{\geq 2}$, and any polynomial $P \in \mathfrak{P}_{k}$, the equality

$$
\int_{L^{\prime}} P(x, y) d \mu_{L}(x, y)=\lambda_{B}(k) a_{p}(k)\left(1-a_{p}(k)^{-2} p^{k-2}\right) \cdot c_{k}^{\sharp}(L)(P)
$$

holds.

Proof. Write $L_{1}$ for $L$, and let $L_{2}$ be any lattice which is contained in $L_{1}$ with index $p$. Then since $L_{1}^{\prime}$ can be expressed as the disjoint union of $L_{1}^{\prime} \cap L_{2}^{\prime}$ and $\frac{1}{p} L_{2}^{\prime} \cap L_{1}^{\prime}$, we have

$$
\begin{aligned}
\int_{L_{1}^{\prime}} P d \mu_{L_{1}} & =\int_{L_{1}^{\prime} \cap L_{2}^{\prime}} P d \mu_{L_{1}}+\int_{\frac{1}{p} L_{2}^{\prime} \cap L_{1}^{\prime}} P d \mu_{L_{1}} \\
& =\int_{L_{1}^{\prime} \cap L_{2}^{\prime}} P d \mu_{L_{1}}+a_{p}(k) \int_{\frac{1}{p} L_{2}^{\prime} \cap L_{1}^{\prime}} P d \mu_{\frac{1}{p} L_{2}},
\end{aligned}
$$


where the last equality follows from Lemma 2.9. By Lemma 2.10,

$$
\int_{L_{1}^{\prime}} P d \mu_{L_{1}}=\lambda_{B}(k)\left(c_{k}\left(L_{1}, L_{2}\right)(P)+a_{p}(k) c_{k}\left(\frac{1}{p} L_{2}, L_{1}\right)(P)\right) .
$$

Equation (49) allows us to rewrite $c_{k}$ in terms of $c_{k}^{\sharp}$, on any pair $\left(L_{1}, L_{2}\right)$ of lattices with $L_{1} \supset L_{2}$ with index $p$ :

$$
c_{k}\left(L_{1}, L_{2}\right)=c_{k}^{\sharp}\left(L_{2}\right)-a_{p}(k)^{-1} c_{k}^{\sharp}\left(p L_{1}\right) .
$$

Proposition 2.11 now follows from (57) and (58), by a direct calculation.

2.5. Forms of weight two and $p$-adic boundary measures. In this section, let $c_{\phi}: \mathcal{E}(\mathcal{T}) \longrightarrow \mathbb{Q}_{p}$ denote the $\tilde{\Gamma}$-invariant function on $\mathscr{E}(\mathcal{T})$ associated to $\phi=\phi_{2} \in S_{2}\left(\Sigma_{0}\left(p N^{+}, N^{-}\right)\right)$. Recall that the form $\phi$ has been normalised so that $\phi$ (and therefore also $c_{\phi}$ ) take values in $\mathbb{Z}$.

Let

$$
\pi: \mathcal{W} \longrightarrow \mathbb{P}_{1}\left(\mathbb{Q}_{p}\right)
$$

denote the natural projection which sends the vector $(x, y)$ to the element $x / y \in \mathbb{P}_{1}\left(\mathbb{Q}_{p}\right)$. If $L$ is any $\mathbb{Z}_{p}$-lattice in $\mathbb{Q}_{p}^{2}$, the restriction of $\pi$ to $L^{\prime}$ has compact fibers and therefore the pushforward of the measure $\mu_{L}$ by $\pi$ is defined, by the usual rule

$$
\int_{X} h(t) d\left(\pi_{*} \mu_{L}\right)(t):=\int_{\pi^{-1}(X)} h(x / y) d \mu_{L}(x, y),
$$

for any compact open $X \subset \mathbb{P}_{1}\left(\mathbb{Q}_{p}\right)$ and any continuous function $h$ on $\mathbb{P}_{1}\left(\mathbb{Q}_{p}\right)$.

Choose any lattice $L$ whose homothety class corresponds to an even vertex of $\mathcal{T}$, and consider the pushforward

$$
\mu_{\phi}:=\pi_{*}\left(\mu_{L}\right) .
$$

Lemma 2.12. The measure $\mu_{\phi}$ is independent of the choice of (even) $L$ that was made to define it.

Proof. Suppose that $L_{1}$ contains $L_{2}$ with index $p$, and that $L_{1}$ corresponds to an even vertex of $\mathcal{T}$, so that $L_{2}$ corresponds to an odd vertex. The region $\mathbb{P}_{1}\left(\mathbb{Q}_{p}\right)$ can be written as a disjoint union

$$
\mathbb{P}_{1}\left(\mathbb{Q}_{p}\right)=\pi\left(L_{1}^{\prime} \cap L_{2}^{\prime}\right) \cup \pi\left(\frac{1}{p} L_{2}^{\prime} \cap L_{1}^{\prime}\right) .
$$


Hence by Lemma 2.9, we have

$$
\begin{array}{ccc}
\pi_{*}\left(\mu_{L_{2}}\right)=a_{p}(2) \pi_{*}\left(\mu_{L_{1}}\right) & \text { on } \quad \pi\left(L_{1}^{\prime} \cap L_{2}^{\prime}\right) ; \\
\pi_{*}\left(\mu_{L_{2}}\right)=a_{p}(2)^{-1} \pi_{*}\left(\mu_{L_{1}}\right) & \text { on } \quad \pi\left(\frac{1}{p} L_{2}^{\prime} \cap L_{1}^{\prime}\right) .
\end{array}
$$

Since $\phi_{2}$ corresponds to a form which is new at $p$, we have $a_{p}(2)= \pm 1$, i.e., $a_{p}(2)=a_{p}(2)^{-1}$. Hence $\pi_{*}\left(\mu_{L_{1}}\right)=a_{p}(2) \pi_{*}\left(\mu_{L_{2}}\right)$. The result follows after noting that $\pi_{*}\left(\mu_{L}\right)$ depends only on the homothety class of $L$, and that $\mathcal{T}$ is connected.

Remark 2.13. The parity restriction in the definition of $\mu_{\phi}$ is only needed when $a_{p}(2)=-1$, which occurs when the modular forms $f$ and $\phi$ correspond to an elliptic curve over $\mathbb{Q}$ with non-split multiplicative reduction at $p$.

Let $\Gamma \subset \mathbf{S L}_{2}\left(\mathbb{Q}_{p}\right)$ denote the subgroup of $\tilde{\Gamma}$ of elements of determinant 1 .

Corollary 2.14. The measure $\mu_{\phi}$ is invariant under the action of $\Gamma$, and satisfies

$$
\mu_{\phi}\left(\mathbb{P}_{1}\left(\mathbb{Q}_{p}\right)\right)=0 .
$$

Proof. Any $\gamma \in \Gamma$ sends the even lattice $L$ to another even lattice $\gamma L$. The first part of the corollary is therefore a direct consequence of Lemmas 2.8 and 2.12. The second follows from Proposition 2.11 in the case $k=2$ and $P=1$.

Let $\mathscr{H}_{p}:=\mathbb{P}_{1}\left(\mathbb{C}_{p}\right)-\mathbb{P}_{1}\left(\mathbb{Q}_{p}\right)$ denote the $p$-adic upper half-plane equipped with its rigid analytic structure. Following [Te], we associate to $\mu_{\phi}$ the rigid analytic function on $\mathscr{H}_{p}$ defined by

$$
f_{\phi}(z):=\int_{\mathbb{P}_{1}\left(\mathbb{Q}_{p}\right)} \frac{d \mu_{\phi}(t)}{(t-z)} .
$$

The fact that $\mu_{\phi}$ is invariant under the action of $\Gamma$ implies that the rigid analytic differential form $\omega_{\phi}:=f_{\phi}(z) d z$ is also invariant under this group, i.e., that $f_{\phi}$ is a modular form of weight two on $\mathscr{H}_{p} / \Gamma$ in the sense of [Da], Sect. 5.2.

As in Sect. 5.3. of [Da], we define the Coleman line integral attached to $\omega_{\phi}$ by the rule

$$
\int_{\tau_{1}}^{\tau_{2}} \omega_{\phi}:=\int_{\mathbb{P}_{1}\left(\mathbb{Q}_{p}\right)} \log \left(\frac{t-\tau_{2}}{t-\tau_{1}}\right) d \mu_{\phi}(t),
$$

where $\log : \mathbb{C}_{p}^{\times} \longrightarrow \mathbb{C}_{p}$ is Iwasawa's branch of the $p$-adic logarithm, satisfying $\log (p)=0$. (Note that one could equally well have chosen any other branch of the $p$-adic logarithm, and in Sect. 2.7 we will see that another 
choice is in fact more appropriate.) This integral satisfies the following properties:

$$
\int_{\tau_{1}}^{\tau_{2}} \omega_{\phi}+\int_{\tau_{2}}^{\tau_{3}} \omega_{\phi}=\int_{\tau_{1}}^{\tau_{3}} \omega_{\phi}
$$

as well as

$$
\int_{\gamma \tau_{1}}^{\gamma \tau_{2}} \omega_{\phi}=\int_{\tau_{1}}^{\tau_{2}} \omega_{\phi}, \quad \text { for all } \gamma \in \Gamma
$$

Since $c_{\phi}$ takes values in $\mathbb{Z}$, Coleman's line integral (61) admits a multiplicative refinement which is more precise and has the virtue of not depending on the choice of a $p$-adic logarithm. It is defined by formally exponentiating the expression for the Coleman line integral given in (61),

$$
\oint_{\tau_{1}}^{\tau_{2}} \omega_{\phi}:=\oint_{\mathbb{P}_{1}\left(\mathbb{Q}_{p}\right)}\left(\frac{t-\tau_{2}}{t-\tau_{1}}\right) d \mu_{\phi}(t),
$$

where the expression on the right is a "multiplicative integral", defined as a limit of Riemann products rather than sums:

$$
f_{\mathbb{P}_{1}\left(\mathbb{Q}_{p}\right)} g(t) d \mu(t):=\lim _{\left\{U_{\alpha}\right\}} \prod_{\alpha} g\left(t_{\alpha}\right)^{\mu\left(U_{\alpha}\right)} .
$$

In this formula, the limit is taken over increasingly fine coverings of $\mathbb{P}_{1}\left(\mathbb{Q}_{p}\right)$ by mutually disjoint compact open subsets $U_{\alpha}$, and the $t_{\alpha}$ are sample points in $U_{\alpha}$. We emphasize that (63) only makes sense if $\mu$ is a $\mathbb{Z}$-valued measure on $\mathbb{P}_{1}\left(\mathbb{Q}_{p}\right)$.

Let $\mathbb{Q}_{p^{2}}$ denote the quadratic unramified extension of $\mathbb{Q}_{p}$, and let $\mathcal{O}_{p^{2}}$ be its ring of integers. Any $\tau$ in $\mathbb{Q}_{p^{2}}-\mathbb{Q}_{p}$ gives rise to a $\mathbb{Z}_{p}$-lattice

$$
L_{\tau}:=\left\{(x, y) \in \mathbb{Q}_{p}^{2} \text { such that } x-\tau y \text { belongs to } \mathcal{O}_{p^{2}}\right\} .
$$

A direct calculation shows that, for all $\gamma=\left(\begin{array}{ll}a & b \\ c & d\end{array}\right) \in \mathbf{S L}_{2}\left(\mathbb{Q}_{p}\right)$,

$$
L_{\gamma \tau}=p^{\operatorname{ord}_{p}(c \tau+d)} \gamma L_{\tau} \text {, }
$$

so that in particular $L_{\gamma \tau}$ and $\gamma L_{\tau}$ are homothetic lattices. In other words, if $v_{\tau} \in \mathcal{T}_{0}$ denotes the vertex of $\mathcal{T}$ associated to $L_{\tau}$, we have

$$
v_{\gamma \tau}=\gamma v_{\tau}, \quad \text { for all } \gamma \in \mathbf{G L}_{2}\left(\mathbb{Q}_{p}\right) .
$$

The set of $\tau \in \mathbb{Q}_{p^{2}}-\mathbb{Q}_{p}$ for which $v_{\tau}$ is an even vertex of $\mathcal{T}$ is called the quadratic unramified upper half-plane, and is denoted $\mathcal{H}_{p}^{\prime}$. Equation (65) shows that $\mathbf{P S L}_{2}\left(\mathbb{Q}_{p}\right)$ acts on $\mathscr{H}_{p}^{\prime}$ by Möbius transformations.

We now record a useful formula for the $p$-adic valuation of the multiplicative Coleman line integral. We do this in the case where the endpoints $\tau_{1}$ 
and $\tau_{2}$ belong to $\mathscr{H}_{p}^{\prime}$. Given an ordered edge $e \in \mathcal{E}(\mathcal{T})$, define the parity function $|e|$ by the rule

$$
|e|= \begin{cases}0 & \text { if } e \text { is even; } \\ 1 & \text { if } e \text { is odd }\end{cases}
$$

and recall that $a_{p}:=a_{p}(2)= \pm 1$.

Proposition 2.15. For all $\tau_{1}, \tau_{2} \in \mathcal{H}_{p}^{\prime}$,

$$
\operatorname{ord}_{p}\left(f_{\tau_{1}}^{\tau_{2}} \omega_{\phi}\right)=\sum_{e: v_{\tau_{1}} \rightarrow v_{\tau_{2}}} a_{p}^{|e|} c_{\phi}(e),
$$

where the sum is taken over the ordered edges in the path joining $v_{\tau_{1}}$ to $v_{\tau_{2}}$. Proof. See Proposition 2.5 of [BDG].

2.6. Indefinite integrals. Given $x \in \mathbb{Q}_{p^{2}}^{\times}$, write $\langle x\rangle$ for the principal unit attached to $x$, defined as the unique element of $1+p \mathcal{O}_{p^{2}}$ satisfying

$$
x=p^{\operatorname{ord}_{p}(x)} \zeta_{x}\langle x\rangle
$$

where $\zeta_{x}$ is a root of unity.

Let $\tau$ be any element of $\mathscr{H}_{p}^{\prime}$. We make the following key definition.

Definition 2.16. The indefinite integral attached to $\tau$ and $\phi$ is defined to be

$$
\int^{\tau} \omega_{\phi}:=\int_{L_{\tau}^{\prime}} \log (x-\tau y) d \mu_{L_{\tau}}(x, y):=\frac{d}{d k}\left(\int_{L_{\tau}^{\prime}}\langle x-\tau y\rangle^{k-2} d \mu_{L_{\tau}}\right)_{k=2} .
$$

We now collect some properties of this indefinite integral. The first of these is a basic $\Gamma$-invariance property.

Proposition 2.17. For all $\gamma \in \Gamma$, and all $\tau \in \mathscr{H}_{p}^{\prime}$,

$$
\int^{\gamma \tau} \omega_{\phi}=\int^{\tau} \omega_{\phi}
$$

Proof. Write $t=\operatorname{ord}_{p}(c \tau+d)$, where $\gamma:=\left(\begin{array}{ll}a & b \\ c & d\end{array}\right)$. By definition,

$$
\begin{aligned}
\int^{\gamma \tau} \omega_{\phi} & =\int_{L_{\gamma \tau}^{\prime}} \log (x-\gamma \tau y) d \mu_{L_{\gamma \tau}}(x, y) \\
& =\int_{p^{t} \gamma L_{\tau}^{\prime}}-\log (c \tau+d)+\log \left(x-\tau y \mid \gamma^{-1}\right) d \mu_{p^{t} \gamma L_{\tau}}(x, y)
\end{aligned}
$$


Note that the first function in this integral is a constant, while the second is periodic under the substitution $(x, y) \mapsto(p x, p y)$. Hence

$$
\begin{aligned}
\int^{\gamma \tau} \omega_{\phi} & =\int_{\gamma L_{\tau}^{\prime}} \log \left(x-\tau y \mid \gamma^{-1}\right) d \mu_{\gamma L_{\tau}}(x, y) \\
& =\int_{L_{\tau}^{\prime}} \log (x-\tau y) d \mu_{L_{\tau}}(x, y)=\int^{\tau} \omega_{\phi},
\end{aligned}
$$

where the last line follows from Lemma 2.8 (which, although it is stated only for integrands which are homogeneous of weight $k-2$ with $k \in U$, extends to the logarithmic integrand appearing in (68) thanks to Definition 2.16).

Recall the Hecke operator $T_{\ell}$ (for $\ell$ a prime, say, not dividing $N$ ) of (37), which gives rise to a correspondence of degree $\ell+1$ on the group $\operatorname{Div}\left(\mathcal{H}_{p} / \Gamma\right)$ of divisors on $\mathscr{H}_{p} / \Gamma$. We extend the indefinite integral by additivity to $\operatorname{Div}\left(\mathscr{H}_{p} / \Gamma\right)$.

Proposition 2.18. For all primes $\ell$ which do not divide $p N^{+} N^{-}$,

$$
\int^{T_{\ell} \tau} \omega_{\phi}=a_{\ell} \int^{\tau} \omega_{\phi}
$$

Proof. By replacing $U$ by a smaller neighborhood, we may assume that its elements are $\equiv 2\left(\bmod p^{2}-1\right)$, so that $\langle x-\tau y\rangle^{k-2}=(x-\tau y)^{k-2}$ on $L_{\tau}^{\prime}$. Hence, by definition of the indefinite integral

$$
\int^{\tau} \omega_{\phi}=\frac{d}{d k}\left(\int_{L_{\tau}^{\prime}}(x-\tau y)^{k-2} d \mu_{L_{\tau}}(x, y)\right) .
$$

On the other hand, the definition of the Hecke operator $T_{\ell}$, combined with the Hecke-equivariance of the specialisation map $\rho_{k}$, shows that

$$
\int^{T_{\ell} \tau} \omega_{\phi}=\frac{d}{d k}\left(\lambda_{B}(k)\left(T_{\ell} c_{k}^{\sharp}\right)\left(L_{\tau}\right)\left((x-\tau y)^{k-2}\right)\right) .
$$

Since $c_{k}^{\sharp}$ is an eigenvector for $T_{\ell}$ with eigenvalue $a_{\ell}(k)$,

$$
\begin{aligned}
\int^{T_{\ell} \tau} \omega_{\phi} & =\frac{d}{d k}\left(\lambda_{B}(k) a_{\ell}(k) c_{k}^{\sharp}\left(L_{\tau}\right)\left((x-\tau y)^{k-2}\right)\right) \\
& =\frac{d}{d k}\left(a_{\ell}(k) \int_{L_{\tau}^{\prime}}(x-\tau y)^{k-2} d \mu_{L_{\tau}}(x, y)\right) .
\end{aligned}
$$

The result follows from (70) and (72) by applying the product rule, after noting that the integral appearing in (72) vanishes at $k=2$.

The next proposition justifies the notation of the indefinite integral by relating it to the Coleman line integral of $\omega_{\phi}$ introduced earlier. 
Proposition 2.19. For all $\tau_{1}, \tau_{2} \in \mathscr{H}_{p}^{\prime}$, with $v_{j}:=v_{\tau_{j}}$, we have

$$
\int^{\tau_{2}} \omega_{\phi}-\int^{\tau_{1}} \omega_{\phi}=\int_{\tau_{1}}^{\tau_{2}} \omega_{\phi}+2 a_{p}^{\prime}(2) \sum_{e: v_{1} \rightarrow v_{2}} a_{p}^{|e|} c_{\phi}(e) .
$$

Remark 2.20. Since $c_{\phi}$ takes values in $\mathbb{Z}$, this formula can be restated in terms of the multiplicative integral attached to $\phi$, in light of Proposition 2.15, as

$$
\int^{\tau_{2}} \omega_{\phi}-\int^{\tau_{1}} \omega_{\phi}=\left(\log +2 a_{p}^{\prime}(2) \operatorname{ord}_{p}\right)\left(f_{\tau_{1}}^{\tau_{2}} \omega_{\phi}\right) .
$$

Before giving the proof of Proposition 2.19 it will be useful to record the following two lemmas.

Lemma 2.21. For all $\alpha \in \Lambda^{\dagger}$, for all $\tau \in \mathcal{H}_{p}^{\prime}$, and for all compact open $X \subset L^{\prime}$,

$$
\int_{X} \log (x-\tau y) d\left(\alpha \mu_{L}\right)=\alpha^{\prime}(2) \mu_{L}(X)+\alpha(2) \int_{X} \log (x-\tau y) d \mu_{L} .
$$

Proof. By definition

$$
\int_{X} \log (x-\tau y) d\left(\alpha \mu_{L}\right)=\frac{d}{d k}\left(\alpha(k) \int_{X}(x-\tau y)^{k-2} d \mu_{L}\right)_{k=2} .
$$

The result follows from the product formula for the derivative appearing in this expression.

Corollary 2.22. If $L_{2}$ is an index $p$ sublattice of $L_{1}$, and $e=\left(\left[L_{1}\right],\left[L_{2}\right]\right)$ denotes the corresponding ordered edge of $\mathcal{T}$, then

$$
\int_{L_{1}^{\prime} \cap L_{2}^{\prime}} \log (x-\tau y) d\left(\mu_{L_{2}}-\mu_{L_{1}}\right)=a_{p}^{\prime}(2) c_{\phi}(e)
$$

if $\phi$ is split multiplicative, and

$\int_{L_{1}^{\prime} \cap L_{2}^{\prime}} \log (x-\tau y) d\left(\mu_{L_{2}}-\mu_{L_{1}}\right)=a_{p}^{\prime}(2) c_{\phi}(e)-2 \int_{L_{1}^{\prime} \cap L_{2}^{\prime}} \log (x-\tau y) d \mu_{L_{1}}$

if $\phi$ is non-split multiplicative.

Proof. By Lemma 2.9,

$$
\int_{L_{1}^{\prime} \cap L_{2}^{\prime}} \log (x-\tau y) d\left(\mu_{L_{2}}-\mu_{L_{1}}\right)=\int_{L_{1}^{\prime} \cap L_{2}^{\prime}} \log (x-\tau y) d\left(\left(a_{p}(k)-1\right) \mu_{L_{1}}\right) .
$$

The result now follows from Lemma 2.21. 
Lemma 2.23. Let $L_{1}$ and $L_{2}$ be even lattices in $\mathbb{Q}_{p}^{2}$ and let $v_{1}$ and $v_{2}$ denote the corresponding vertices of $\mathcal{T}$. Then

$$
\int_{W} \log (x-\tau y) d\left(\mu_{L_{2}}-\mu_{L_{1}}\right)(x, y)=2 a_{p}^{\prime}(2) \sum_{e: v_{1} \rightarrow v_{2}} a_{p}^{|e|} c_{\phi}(e) .
$$

Proof. We treat the case where $\phi$ is split multiplicative so that $a_{p}(2)=1$. Assume first that $L_{1}$ contains $L_{2}$ with index $p$, and that $v_{1}$ is even (so that the adjacent vertex $v_{2}$ is odd.) Let $e$ be the ordered edge of $\mathcal{T}$ having $v_{1}$ as source and $v_{2}$ as target. The measure $\mu_{L_{1}}-\mu_{L_{2}}$ is supported on $L_{1}^{\prime} \cup L_{2}^{\prime}$, which can be expressed as a disjoint union

$$
L_{1}^{\prime} \cup L_{2}^{\prime}=\left(L_{1}^{\prime} \cap L_{2}^{\prime}\right) \cup\left(\frac{1}{p} L_{2}^{\prime} \cap L_{1}^{\prime}\right) \cup\left(L_{2}^{\prime} \cap p L_{1}^{\prime}\right) .
$$

By Corollary 2.22,

$$
\int_{L_{1}^{\prime} \cap L_{2}^{\prime}} \log (x-\tau y) d\left(\mu_{L_{2}}-\mu_{L_{1}}\right)(x, y)=a_{p}^{\prime}(2) c_{\phi}(e) .
$$

Furthermore, we note that the sets $\frac{1}{p} L_{2}^{\prime} \cap L_{1}^{\prime}$ and $\left(L_{2}^{\prime} \cap p L_{1}^{\prime}\right)$ are disjoint from the supports of the measures $\mu_{L_{2}}$ and $\mu_{L_{1}}$ respectively. Therefore

$$
\begin{aligned}
\int_{\frac{1}{p} L_{2}^{\prime} \cap L_{1}^{\prime}} \log (x-\tau y) d\left(\mu_{L_{2}}-\mu_{L_{1}}\right) & =-\int_{\frac{1}{p} L_{2}^{\prime} \cap L_{1}^{\prime}} \log (x-\tau y) d \mu_{L_{1}} \\
& =-\int_{L_{2}^{\prime} \cap p L_{1}^{\prime}} \log (x-\tau y) d \mu_{p L_{1}}, \\
\int_{L_{2}^{\prime} \cap p L_{1}^{\prime}} \log (x-\tau y) d\left(\mu_{L_{2}}-\mu_{L_{1}}\right) & =\int_{L_{2}^{\prime} \cap p L_{1}^{\prime}} \log (x-\tau y) d \mu_{L_{2}} .
\end{aligned}
$$

The result now follows by adding together the expressions for each of the three contributions given in (74), (76), and (77), invoking Corollary 2.22 to further simplify the sum of (76) and (77).

We are now ready to prove Proposition 2.19.

Proof. Set $L_{j}:=L_{\tau_{j}}$ for $j=1,2$. Observe that

$$
\begin{aligned}
\int^{\tau_{2}} \omega_{\phi}-\int^{\tau_{1}} \omega_{\phi}= & \int_{W} \log \left(\frac{x-\tau_{2} y}{x-\tau_{1} y}\right) d \mu_{L_{1}}(x, y) \\
& +\int_{W} \log \left(x-\tau_{2} y\right) d\left(\mu_{L_{2}}-\mu_{L_{1}}\right)(x, y) .
\end{aligned}
$$

The first integral appearing in the right hand side of (78) involves a function which is constant along the fibers of $\pi$. This integral is therefore equal to

$$
\int_{\mathbb{P}_{1}\left(\mathbb{Q}_{p}\right)} \log \left(\frac{t-\tau_{2}}{t-\tau_{1}}\right) d \mu_{\phi}(t)=\int_{\tau_{1}}^{\tau_{2}} \omega_{\phi}
$$


As for the second integral appearing in the right hand side of (78), Lemma 2.23 shows that

$$
\int_{W} \log \left(x-\tau_{2} y\right) d\left(\mu_{L_{2}}-\mu_{L_{1}}\right)(x, y)=2 a_{p}^{\prime}(2) \sum_{e: v_{1} \rightarrow v_{2}} a_{p}^{|e|} c_{\phi}(e) .
$$

The proof of Proposition 2.19 follows.

2.7. Elliptic curves and the $\mathcal{L}$-invariant. This section presents an exposition of the material developed in [BDI] in the more general setting of modular forms of arbitrary even weight, specialised to the setting of weight two. This is done for completeness, and for the convenience of the reader, because the weight two setting can be treated with less machinery. Notably the "locally analytic distributions" required in [BDI] are avoided in the more elementary treatment of the weight two situation given here.

The assumption that the elliptic curve $E$ of the Introduction has at least one prime $\neq p$ of multiplicative reduction allows us to write its conductor as $N=p N^{+} N^{-}$, whose factors satisfy the hypotheses of (32).

We now give a formula for the $\mathcal{L}$-invariant of $E$ in terms of the multiplicative line integrals attached to $\omega_{\phi}$, arising from the $p$-adic uniformisation theory of Cerednik and Drinfeld. Let $Q$ be an infinite discrete subgroup of $\mathbb{Q}_{p}^{\times}$. The slope of $Q$ is defined by choosing an element $q \in Q$ with $\operatorname{ord}_{p}(q) \neq 0$, and setting

$$
\mathcal{L}_{Q}:=\frac{\log (q)}{\operatorname{ord}_{p}(q)}
$$

Note that

(1) The slope of $Q$ does not depend on the choice of element $q$ that was made to define it, and in fact depends only on the commensurability class of the lattice $Q$.

(2) If $E$ is an elliptic curve having multiplicative reduction at $p$, and $Q_{E}$ is its lattice of Tate periods, then by definition

$$
\mathcal{L}_{E}=\mathcal{L}_{Q_{E}}
$$

is the $\mathcal{L}$-invariant attached to $E$.

Theorem 2.24. Let $Q_{\phi}$ be the multiplicative subgroup of periods of the rigid analytic differential $\omega_{\phi}$, defined by

$$
Q_{\phi}:=\left\{f_{\tau}^{\gamma \tau} \omega_{\phi} \text { such that } \tau \in \mathscr{H}_{p}^{\prime} \text {, and } \gamma \in \Gamma\right\} .
$$

Then $Q_{\phi}$ is a lattice in $\mathbb{Q}_{p}^{\times}$which is commensurable with $Q_{E}$. In particular,

$$
\mathcal{L}_{Q_{\phi}}=\mathscr{L}_{E} .
$$


Proof. This follows from the Cerednik-Drinfeld theory of $p$-adic uniformisation of Shimura curves, as it is explained for example in [GvdP]. Some more details are also given in Sect. 4.2.

We use the notions that have been developed so far to give a new proof of (5) of the introduction. This equation was originally proved by Greenberg and Stevens, by a different approach.

Theorem 2.25. $\mathcal{L}_{E}=-2 a_{p}^{\prime}(2)$.

Proof. Choose an element $\gamma \in \Gamma$ in such a way that the associated multiplicative period

$$
q_{\gamma}:=f_{\tau}^{\gamma \tau} \omega_{\phi}
$$

generates a lattice in $\mathbb{Q}_{p}^{\times}$. By Theorem 2.24,

$$
\mathcal{L}_{E}=\frac{\log \left(q_{\gamma}\right)}{\operatorname{ord}_{p}\left(q_{\gamma}\right)} .
$$

Now we observe that

(1) By Proposition 2.17,

$$
\int^{\gamma \tau} \omega_{\phi}-\int^{\tau} \omega_{\phi}=0
$$

(2) On the other hand, by (73),

$$
\int^{\gamma \tau} \omega_{\phi}-\int^{\tau} \omega_{\phi}=\log \left(q_{\gamma}\right)+2 a_{p}^{\prime}(2) \operatorname{ord}_{p}\left(q_{\gamma}\right) .
$$

By combining these two facts it follows that

$$
\frac{\log \left(q_{\gamma}\right)}{\operatorname{ord}_{p}\left(q_{\gamma}\right)}=-2 a_{p}^{\prime}(2) .
$$

Theorem 2.25 now follows from (79) and (81).

Corollary 2.26. If $\log _{q}$ denotes the branch of the p-adic logarithm which vanished on $q$, where $Q_{E}=\langle q\rangle$ modulo torsion. Then

$$
\int^{\tau_{2}} \omega_{\phi}-\int^{\tau_{1}} \omega_{\phi}=\log _{q}\left(f_{\tau_{1}}^{\tau_{2}} \omega_{\phi}\right) .
$$

Proof. This follows from (73) and (81).

\section{Hida $p$-adic $L$-functions attached to imaginary quadratic fields}

This chapter attaches a $p$-adic $L$-function to the Hida family $\phi_{\infty}$ and to an imaginary quadratic field $K$, using the system of measures $\left\{\mu_{L}\right\}$ constructed in Sect. 2. 
3.1. Optimal embeddings and special values. Let $N=p N^{+} N^{-}$be the factorization introduced in (32). Recall that the factors $p, N^{+}$and $N^{-}$are pairwise relatively prime, and that $N^{-}$is a square-free product of an odd number of prime factors. We maintain the notations of Sect. 2. Thus $B$ denotes the definite quaternion algebra of discriminant $N^{-} \infty$, and $R$ is a fixed Eichler $\mathbb{Z}[1 / p]$-order of level $N^{+}$in $B$. We also fix an Eichler $\mathbb{Z}$-order $\underline{R}$ of $B$ of level $N^{+}$, choosing it in such a way that

$$
\underline{R}[1 / p]=R .
$$

As usual let $\underline{\hat{R}}:=\underline{R} \otimes \hat{\mathbb{Z}}$ denote the adelisation of $\underline{R}$.

Definition 3.1. An imaginary quadratic field $K$ is said to be admissible relative to the factorisation (32) if it satisfies

(1) All primes dividing $N^{+}$, resp. $N^{-}$are split, resp. inert in $K$.

(2) The prime $p$ is unramified in $K$.

Fix an admissible imaginary quadratic field $K$, of discriminant $D<0$ say. Let $\mathcal{O}_{K}$ and $\mathcal{O}:=\mathcal{O}_{K}[1 / p]$ denote its ring of integers and $p$-integers respectively. Denote by $\operatorname{hom}(K, B)$ the set of $\mathbb{Q}$-algebra homomorphisms from $K$ to $B$.

Definition 3.2. An optimal embedding of $K$ into $B$ of level $N^{+}$is a pair $(\Psi, b) \in \operatorname{hom}(K, B) \times\left(\hat{B}^{\times} / \underline{\hat{R}}^{\times}\right)$satisfying

$$
\Psi(K) \cap\left(b \underline{\hat{R}} b^{-1}\right)=\Psi\left(\mathcal{O}_{K}\right) .
$$

The assumption that $K$ is admissible implies the existence of such optimal embeddings. More precisely, the condition in Definition 3.1 that all the primes dividing $N^{-}$are inert in $K$ implies that the $\operatorname{set} \operatorname{hom}(K, B)$ is non-empty. Given $\Psi \in \operatorname{hom}(K, B)$, the possibility of producing $b \in \hat{B}^{\times}$ satisfying (82) is then guaranteed by the theory of local embeddings, in light of the assumption that the primes dividing $N^{+}$are split in $K$. See Sect. 3 of [Gr] for more details.

Occasionally we commit an abuse of notation and use the symbol $\Psi$ to denote the optimal embedding $(\Psi, b)$, omitting the extra datum of $b \in \hat{B}^{\times}$ when this results in no ambiguity. It should be kept in mind that such a $b$ is always part of the data associated to an optimal embedding, even if it is suppressed from the notation in order to lighten it.

Any optimal embedding $\Psi=(\Psi, b)$ gives rise to a pair $\left(\Psi, \underline{R}_{b}\right)$ where $\underline{R}_{b}$ is the Eichler order of level $N^{+}$in $B$ determined from $b$ by the rule

$$
\underline{R}_{\Psi}=\left(b \underline{\hat{R}} b^{-1}\right) \cap B,
$$

so that $\Psi$ is an embedding of $\mathcal{O}_{K}$ into $\underline{R}_{b}$. 
The group $B^{\times}$acts on $\operatorname{hom}(K, B)$ on the left by conjugation, and on $\left(\hat{B}^{\times} / \underline{\hat{R}}^{\times}\right)$by left multiplication. The resulting diagonal action of $B^{\times}$on the cartesian product hom $(K, B) \times\left(\hat{B}^{\times} / \underline{\hat{R}}^{\times}\right)$defined by

$$
g \cdot(\Psi, b)=\left(g \Psi g^{-1}, g b\right)
$$

preserves the collection of optimal embeddings of level $N^{+}$. Let

$$
\operatorname{Emb}\left(\mathcal{O}_{K}, N^{+}, N^{-}\right) \subset B^{\times} \backslash\left(\operatorname{hom}(K, B) \times\left(\hat{B}^{\times} / \underline{\hat{R}}^{\times}\right)\right)
$$

be the set of orbits for this action. Given an optimal embedding $(\Psi, b)$, the notation $[\Psi, b]$, or sometimes just $[\Psi]$, will be used to denote the associated element of $\operatorname{Emb}\left(\mathcal{O}_{K}, N^{+}, N^{-}\right)$.

The class group $G_{D}$ of $K$, which is described adelically as the quotient

$$
G_{D}:=\hat{K}^{\times} / K^{\times} \hat{\mathcal{O}}_{K}^{\times},
$$

acts naturally on $\operatorname{Emb}\left(\mathcal{O}_{K}, N^{+}, N^{-}\right)$by the rule

$$
\sigma \cdot[\Psi, b]=[\Psi, \hat{\Psi}(\sigma) b],
$$

where $\hat{\Psi}$ denotes the map from $\hat{K}$ to $\hat{B}$ induced from $\Psi$ by tensoring with $\hat{\mathbb{Z}}$.

Associated to any $\Psi \in \operatorname{hom}(K, B)$ is the binary quadratic form $Q_{\Psi}(x, y)$ of discriminant $4 D$ with coefficients in $\mathbb{Q}_{p}$ defined by

$$
Q_{\Psi}(x, y)=c x^{2}+(d-a) x y-b y^{2}, \quad \text { where }\left(\begin{array}{ll}
a & b \\
c & d
\end{array}\right):=\iota_{p} \Psi(\sqrt{D}) .
$$

Note that $Q_{\Psi}$ coincides with the polynomial $P_{\Psi(\sqrt{D})}$ attached to the trace zero element $\Psi(\sqrt{D})$, defined in (50). This quadratic form is determined, up to sign, by its discriminant and the fact that

$$
Q_{\Psi}\left(\tau_{\Psi}, 1\right)=Q_{\Psi}\left(\tau_{\Psi}^{\prime}, 1\right)=0,
$$

where $\tau_{\Psi}$ and $\tau_{\Psi}^{\prime}$ are the fixed points for the action of $\iota_{p} \Psi\left(K_{p}^{\times}\right)$on $\mathbb{P}_{1}\left(\mathbb{C}_{p}\right)$ by Möbius transformations. Thus, $Q_{\Psi}(x, y)=0$ if and only if $(x, y)$ is either the zero vector or an eigenvector for this torus action. This remark makes it apparent that for any $g \in B^{\times}$, we have

$$
Q_{g \Psi g^{-1}}=\operatorname{det}(g)\left(Q_{\Psi} \mid g^{-1}\right),
$$

where $\operatorname{det}(g):=\operatorname{det}\left(\iota_{p}(g)\right)$ is the reduced norm on $B$.

The fixed points $\tau_{\Psi}$ and $\tau_{\Psi}^{\prime}$ of $\iota_{p} \Psi\left(K^{\times}\right)$acting on $\mathbb{P}_{1}\left(\mathbb{C}_{p}\right)$ play an important role, and it is convenient to make a consistent ordering of these by fixing an embedding of $K$ into $\mathbb{C}_{p}$ and requiring that

$$
\iota_{p} \Psi(\alpha)\left(\begin{array}{c}
\tau_{\Psi} \\
1
\end{array}\right)=\alpha\left(\begin{array}{c}
\tau_{\Psi} \\
1
\end{array}\right) .
$$


The assignment (84) makes it possible to associate to the weight $k$ newform $\phi_{k}^{\sharp} \in S_{k}\left(N^{+}, N^{-}\right)$and to each optimal embedding $\Psi=(\Psi, b)$ of level $N^{+}$a numerical invariant $\phi_{k}^{\sharp}[\Psi]$ by the rule

$$
\phi_{k}^{\sharp}[\Psi]:=\operatorname{det}\left(b_{p}\right)^{1-k / 2} \phi_{k}^{\sharp}(b)\left(Q_{\Psi}^{\frac{k-2}{2}} \mid b_{p}\right),
$$

where $b_{p} \in B_{p}$ denotes the component at $p$ of the idèle $b$. A direct verification shows that $\phi_{k}^{\sharp}[\Psi]$ depends only on the class of $\Psi$ in $\operatorname{Emb}\left(\mathcal{O}_{K}, N^{+}, N^{-}\right)$ and hence is a well-defined function on this quotient set.

Likewise, if $p$ is split in $K$, then the set $\operatorname{Emb}\left(\mathcal{O}_{K}, p N^{+}, N^{-}\right)$is nonempty and we can thus associate to $[\Psi, b]$ and $\phi_{2}$ the well-defined numerical invariant

$$
\phi_{2}[\Psi]:=\phi_{2}(b) .
$$

We now introduce further notations that are better adapted to the $p$-adic calculations developed in the next two sections.

The $\mathbb{Z}[1 / p]$-order $R$ in $B$ satisfies the Eichler condition, and hence, by strong approximation, any optimal embedding of $K$ of level $N^{+}$can be translated on the left by an element of $B^{\times}$so that it lies in $\operatorname{hom}(\mathcal{O}, R) \times\left(\hat{R}^{\times} / \underline{\hat{R}}^{\times}\right)$. This makes it possible to identify $\operatorname{Emb}\left(\mathcal{O}_{K}, N^{+}, N^{-}\right)$with a subset of

$$
R^{\times} \backslash\left(\operatorname{hom}(\mathcal{O}, R) \times\left(\mathbf{G L}_{2}\left(\mathbb{Q}_{p}\right) / \mathbf{G L}_{2}\left(\mathbb{Z}_{p}\right)\right)\right) .
$$

Given $[\Psi] \in \operatorname{Emb}\left(\mathcal{O}_{K}, N^{+}, N^{-}\right)$, denote by $[\Psi, g]$ with $g \in \mathbf{G L}_{2}\left(\mathbb{Q}_{p}\right)$ any representative of $[\Psi]$ lying in (88). The resulting pair $(\Psi, g)$ is entirely determined by the embedding $\Psi$ together with the lattice $L_{\Psi}:=g\left(\mathbb{Z}_{p}^{2}\right)$ so that it is convenient to label $[\Psi]$ by such data and write $\left[\Psi, L_{\Psi}\right]$ for $[\Psi]$. The optimality condition satisfied by $\Psi$ implies that the lattice $L_{\Psi}$ is preserved under the action of $\iota_{p} \Psi\left(\mathcal{O}_{K}\right)$. Note also that, since the scalar $p$ belongs to $R^{\times}$, we have $\left[\Psi, L_{\Psi}\right]=\left[\Psi, p L_{\Psi}\right]$.

A similar discussion applies for $\operatorname{Emb}\left(\mathcal{O}_{K}, p N^{+}, N^{-}\right)$, which can be identified with a subset of

$$
R^{\times} \backslash\left(\operatorname{hom}(\mathcal{O}, R) \times \mathbf{G L}_{2}\left(\mathbb{Q}_{p}\right) / \Gamma_{0}\left(p \mathbb{Z}_{p}\right)\right) .
$$

An element $[\Psi]=[\Psi, g]$ of this space can thus be labelled by a triple [ $\left.\Psi, L_{1}, L_{2}\right]$, where the $\mathbb{Z}_{p}$-lattices $L_{1}$ and $L_{2}$, defined by $L_{1}:=g\left(\mathbb{Z}_{p}^{2}\right)$ and $L_{2}:=g\left(\mathbb{Z}_{p} \oplus p \mathbb{Z}_{p}\right)$, are both preserved under the action $\iota_{p} \Psi\left(\Gamma_{0}\left(p \mathbb{Z}_{p}\right)\right)$. It shall sometimes be convenient to describe optimal embeddings of level $p N^{+}$in this way.

The invariants $\phi_{k}^{\sharp}[\Psi]$ and $\phi_{2}[\Psi]$ can be expressed in terms of the lattice functions $c_{k}^{\sharp}(L)$ and $c_{2}\left(L_{1}, L_{2}\right)$ of Sects. 2.2 and 2.3 by the formulae

$$
\begin{aligned}
\phi_{k}^{\sharp}\left[\Psi, L_{\Psi}\right] & =\left|L_{\Psi}\right|^{1-k / 2} c_{k}^{\sharp}\left(L_{\Psi}\right)\left(Q_{\Psi}^{\frac{k-2}{2}}\right), \\
\phi_{2}\left[\Psi, L_{1}, L_{2}\right] & =c_{2}\left(L_{1}, L_{2}\right),
\end{aligned}
$$

where $|L|$ is the generalised index of the lattice $L$ in $\mathbb{Z}_{p}^{2}$. 
Let $h$ denote the class number of $K$ and let

$$
G_{D}:=\left\{\sigma_{1}, \ldots, \sigma_{h}\right\}
$$

be a complete list of elements of $G_{D}$, with $\sigma_{1}=1$. Choose any element $[\Psi]:=[\Psi, b] \in \operatorname{Emb}\left(\mathcal{O}_{K}, N^{+}, N^{-}\right)$and write

$$
\left[\Psi^{\sigma_{j}}\right]=\left[\Psi^{\sigma_{j}}, b^{\sigma_{j}}\right]:=\sigma_{j} \cdot[\Psi, b] .
$$

The importance of the optimal embeddings and the invariants $\phi_{k}^{\sharp}[\Psi]$ lies in their relation with the central critical values of $L$-series attached to $f_{k}^{\sharp}$ and to abelian characters of $\operatorname{Gal}(\bar{K} / K)$. We restrict our attention to the quadratic characters of the class group $G_{D}$. These are called genus characters. If $\chi$ is a non-trivial genus character, it cuts out a quadratic extension of $K=\mathbb{Q}(\sqrt{D})$ which is a biquadratic extension of the form

$$
K_{\chi}=\mathbb{Q}\left(\sqrt{D_{1}}, \sqrt{D_{2}}\right), \quad \text { with } D=D_{1} D_{2} .
$$

In fact, the genus characters of $K$ are in bijection with the factorisations of $D$ into a product of two fundamental discriminants $D_{1}$ and $D_{2}$, with the trivial character corresponding to the factorisation $D_{1}=1, D_{2}=D$. Let $\chi_{1}, \chi_{2}$ and $\epsilon_{K}$ denote the Dirichlet characters attached to the discriminants $D_{1}, D_{2}$ and $D$ respectively. Then $\chi_{1} \chi_{2}=\epsilon_{K}$. The $L$-series $L\left(f_{k}^{\sharp} / K, \chi, s\right)$ is defined by the usual Euler product expansion whose Euler factors at the good primes are of degree 4 . This definition makes it apparent that

$$
L\left(f_{k}^{\sharp} / K, \chi, s\right)=L\left(f_{k}^{\sharp}, \chi_{1}, s\right) L\left(f_{k}^{\sharp}, \chi_{2}, s\right) .
$$

Assume from now on in the paper that $D \neq-3,-4$, so that $\mathcal{O}_{K}^{\times}=\{ \pm 1\}$. This condition can always be ensured in the arguments of the next sections. The algebraic part of $L\left(f_{k}^{\sharp} / K, \chi, k / 2\right)$ is defined as follows:

$$
L^{*}\left(f_{k}^{\sharp} / K, \chi, k / 2\right):=\frac{(k / 2-1) !^{2} D^{\frac{k-1}{2}}}{(2 \pi)^{k-2}\left\langle f_{k}^{\sharp}, f_{k}^{\sharp}\right\rangle} L\left(f_{k}^{\sharp} / K, \chi, k / 2\right),
$$

where $\left\langle f_{k}^{\sharp}, f_{k}^{\sharp}\right\rangle$ denotes as before the Petersson scalar product of $f_{k}^{\sharp}$ with itself. Likewise for $k=2$ we write

$$
L^{*}(f / K, \chi, 1):=\frac{\sqrt{D}}{\langle f, f\rangle} L(f / K, \chi, 1),
$$

Proposition 3.3 (Hatcher, Hui Xue). For all $k \in U \cap \mathbb{Z}^{>2}$,

$$
L^{*}\left(f_{k}^{\sharp} / K, \chi, k / 2\right)=\left(\sum_{j=1}^{h} \chi\left(\sigma_{j}\right) \phi_{k}^{\sharp}\left[\Psi^{\sigma_{j}}\right]\right)^{2} .
$$


Proof. This result follows from the one that is given in [Ha1] and [Ha2] in the special case where $N^{-}$is prime and $N^{+}=1$. For the convenience of the reader, we briefly explain how to translate the current formalism into the one used in [Ha1]; see also [BDIS], pp. 435-436. Hatcher considers the finite dimensional Hecke-module

$$
\mathcal{V}=\left\{v: \underline{\hat{R}}^{\times} \backslash \hat{B}^{\times} \longrightarrow \mathfrak{P}_{k}: v(b g)=v(b) \cdot \iota_{p}(g), \text { for all } g \in B^{\times}\right\} .
$$

To any optimal embedding $(\Psi, b)$ of level one, we attach the natural image of the pair $\left(b, Q_{\Psi}(x, y)^{k / 2-1}\right)$ in $\mathcal{V}$, say $v_{\Psi}$. Set

$$
v_{\chi}:=\sum_{j=1}^{h} \chi\left(\sigma_{j}\right) \nu_{\Psi_{j}^{\sigma}} \in \mathcal{V} .
$$

Then, Hatcher's result states the equality

$$
L^{*}\left(f_{k}^{\sharp} / K, \chi, k / 2\right)=\left\langle v_{\chi, f_{k}^{\sharp}}, v_{\chi, f_{k}^{\sharp}}\right\rangle_{k},
$$

where $v_{\chi, f_{k}^{\sharp}}$ denotes the $f_{k}^{\sharp}$-isotypic projection of $v_{\chi}$, and $\langle,\rangle_{k}$ denotes the natural perfect pairing on $\mathcal{V}$ induced by the scalar product of (51). In view of (90), and of the normalisation condition $\left\langle f_{k}^{\sharp}, f_{k}^{\sharp}\right\rangle=1, v_{\chi, f_{k}^{\sharp}}$ can be written as $\left(\sum_{j=1}^{h} \chi\left(\sigma_{j}\right) \phi_{k}^{\sharp}\left[\Psi^{\sigma_{j}}\right]\right) \cdot v_{f_{k}^{\sharp}}$, where $v_{f_{k}^{\sharp}}$ is a normalised eigenvector for the action of the Hecke algebra on $\mathcal{V}$ via the character attached to $f_{k}^{\sharp}$. This explains our special value formula in the setting of Hatcher's paper. Work of Hui Xue provides an extension of Hatcher's formula to the level of generality that is required. Cf. Theorem 3 of [HX1] (and also [HX2]), generalizing the results of [Gr] and [Ha1], [Ha2].

When $k=2$, it is necessary to work with the newforms $f_{2}$ and $\phi_{2}$ of level $p N^{+} N^{-}$. The set $\operatorname{Emb}\left(\mathcal{O}_{K}, p N^{+}, N^{-}\right)$is non-empty if and only if $p$ is split in $K$. In this case, choose the elements $\left[\Psi^{\sigma_{j}}\right]$ as in (92), but starting this time with $[\Psi, b] \in \operatorname{Emb}\left(\mathcal{O}_{K}, p N^{+}, N^{-}\right)$.

Proposition 3.4. If $p$ is inert in $K$, then $L^{*}(f / K, \chi, 1)=0$. If $p$ is split in $K$,

$$
L^{*}(f / K, \chi, 1)=\left\langle\phi_{2}, \phi_{2}\right\rangle^{-1}\left(\sum_{j=1}^{h} \chi\left(\sigma_{j}\right) \phi_{2}\left[\Psi^{\sigma_{j}}\right]\right)^{2} .
$$

Proof. The first statement follows from the fact that the sign in the functional equation for $L(f / K, \chi, s)$ is -1 when $p$ is inert in $K$. The formula for $p$ split follows from Proposition 3.3 by replacing $N^{+}$by $p N^{+}$and specialising to $k=2$, noting that the form $\phi_{2}$, unlike $\phi_{k}^{\sharp}$, has not been normalised to be of length 1 . 
3.2. Two-variable $p$-adic $L$-functions. Let $[\Psi]$ be a class of optimal embeddings of level $N^{+}$, represented by an element $\left[\Psi, L_{\Psi}\right]$ of the space (88).

If $p$ is inert in $K$, then the lattice $L_{\Psi}$ is determined up to homothety by the requirement that it be preserved under the action of the non-split torus $\iota_{p} \Psi\left(K_{p}^{\times}\right)$.

If $p$ is split in $K$, then the lattice $L_{\Psi}$ is stable under the action of the split quadratic algebra $\iota_{p} \Psi\left(\mathcal{O}_{K} \otimes \mathbb{Z}_{p}\right)$, and hence admits a $\mathbb{Z}_{p}$-basis $\left(v_{1}, v_{2}\right)$ consisting of eigenvectors for the action of this algebra.

Define a region $L_{\Psi}^{\prime \prime}$ in $L_{\Psi}^{\prime}$ by the rule

$$
L_{\Psi}^{\prime \prime}:= \begin{cases}L_{\Psi}^{\prime} & \text { if } p \text { is inert in } K \\ \mathbb{Z}_{p}^{\times} v_{1} \times \mathbb{Z}_{p}^{\times} v_{2} & \text { if } p \text { is split in } K .\end{cases}
$$

Definition 3.5. (1) The partial $p$-adic $L$-function associated to $f_{\infty}$ and [ $\left.\Psi\right]$ is the analytic function of the variable $k \in U$ defined by

$$
\mathcal{L}_{p}\left(f_{\infty} / K, \Psi, k\right):=\int_{L_{\Psi}^{\prime \prime}}\left\langle Q_{\Psi}(x, y)\right\rangle^{\frac{k-2}{2}} d \mu_{L \Psi}(x, y) .
$$

(2) The $p$-adic $L$-function attached to $f_{\infty}$ and the quadratic character $\chi$ of $G_{D}$ is the analytic function of $k \in U$ defined by

$$
L_{p}\left(f_{\infty} / K, \chi, k\right)=\left(\mathscr{L}_{p}\left(f_{\infty} / K, \chi, k\right)\right)^{2},
$$

where

$$
\mathcal{L}_{p}\left(f_{\infty} / K, \chi, k\right):=\sum_{j=1}^{h} \chi\left(\sigma_{j}\right) \mathcal{L}_{p}\left(f_{\infty} / K, \Psi^{\sigma_{j}}, k\right) .
$$

Remark 3.6. In this remark, assume for simplicity that $h=1$. Motivated by Definition 1.11, it is tempting to associate to $f_{\infty}$ and $K$ a two-variable $p$-adic $L$-function of the variables $(k, s) \in U \times \mathbb{Z}_{p}$ by introducing the factorisation

$$
Q_{\Psi}(x, y)=A\left(x-\tau_{\Psi} y\right)\left(x-\tau_{\Psi}^{\prime} y\right)
$$

of $Q_{\Psi}$ into a product of two linear forms, and setting

$$
\begin{aligned}
& \mathcal{L}_{p}\left(f_{\infty} / K, k, s\right)=A^{\frac{k-2}{2}} \int_{L_{\Psi}^{\prime \prime}}\left\langle x-\tau_{\Psi} y\right\rangle^{s-1}\left\langle x-\tau_{\Psi}^{\prime} y\right\rangle^{k-s-1} d \mu_{L_{\Psi}}(x, y) \\
& L_{p}\left(f_{\infty} / K, k, s\right)=\mathcal{L}_{p}\left(f_{\infty} / K, k, s\right) \mathcal{L}_{p}\left(f_{\infty} / K, k, k-s\right)
\end{aligned}
$$

This two-variable $L$-function specialises to the function $L_{p}\left(f_{\infty} / K, \chi_{\text {triv }}, k\right)$ when restricted to the central critical line $s=k / 2$. Its restriction to the "weight two" line $k=2$ is the anticyclotomic $p$-adic $L$-function explored in [BD1] and [BD2], which interpolates the special values of the $L$-function of $E$ over $K$ at $s=1$, twisted by ring class characters of $K$ of $p$-power conductor. It is only the function $L_{p}\left(f_{\infty} / K, \chi, k\right)$ that plays a role in the constructions of the present paper, although it is clear that a systematic study of 
the leading term of $L_{p}\left(f_{\infty} / K, k, s\right)$ at $(k, s)=(2,1)$ would be worthwhile. For instance, in the case where $p$ is inert, the methods of [BD2] are exploited in Sect. 3.4 to relate this leading term to Heegner points. This strengthens the main result of [BD2] concerning the anticyclotomic $p$-adic $L$-function $L_{p}\left(f_{\infty} / K, 2, s\right)$, and places this result in a broader and more natural setting.

We close this section by recording an elementary property of the subsets $L_{\Psi}^{\prime \prime}$.

Lemma 3.7. The function $\operatorname{ord}_{p}\left(Q_{\Psi}(x, y)\right)$ is constant on $L_{\Psi}^{\prime \prime}$. More precisely,

$$
\operatorname{ord}_{p}\left(Q_{\Psi}(x, y)\right)=\operatorname{ord}_{p}\left(\left|L_{\Psi}\right|\right) \quad \text { on } L_{\Psi}^{\prime \prime} .
$$

Proof. If $p$ is split in $K$, then the quadratic form $Q_{\Psi}$ can be factored as a product of two linear forms $q_{1}(x, y)$ and $q_{2}(x, y)$ satisfying

$$
q_{1}\left(v_{1}\right)=q_{2}\left(v_{2}\right)=0 \text {. }
$$

For any $v=a v_{1}+b v_{2} \in L_{\Psi}^{\prime \prime}$, with $a, b \in \mathbb{Z}_{p}^{\times}$, we have

$$
Q_{\Psi}\left(a v_{1}+b v_{2}\right)=a b q_{1}\left(v_{2}\right) q_{2}\left(v_{1}\right) .
$$

The independence of $\operatorname{ord}_{p}\left(Q_{\Psi}\right)$ follows. The more precise formula (95) can be checked by noting that it is invariant under conjugating the embedding $\iota_{p}$, and then verifying it in the case where $\iota_{p} \Psi$ gives rise to the diagonal embedding of $K_{p}=\mathbb{Q}_{p} \times \mathbb{Q}_{p}$ into $M_{2}\left(\mathbb{Q}_{p}\right)$ and $L_{\Psi}=\mathbb{Z}_{p}^{2}$, so that $Q_{\Psi}=2 \sqrt{D} x y$, and $\left(v_{1}, v_{2}\right)$ can be chosen to be the standard basis $(1,0),(0,1)$. The proof when $p$ is inert in $K$ proceeds along similar, but simpler, lines, and is left to the reader.

3.3. The interpolation formula. This section further justifies the designation of $p$-adic $L$-function that is given to $L_{p}\left(f_{\infty} / K, \chi, k\right)$ by giving an interpolation formula relating this $p$-adic $L$-function to classical $L$-values.

Recall (93) defining the algebraic part of the special value of the $L$-series $L\left(f_{k}^{\sharp} / K, \chi, k / 2\right)$.

Theorem 3.8. Assume that $p$ is inert in $K$. Then $L_{p}\left(f_{\infty} / K, \chi, 2\right)=0$, and for all $k \in U \cap \mathbb{Z}^{>2}$,

$$
L_{p}\left(f_{\infty} / K, \chi, k\right)=\lambda_{B}(k)^{2} a_{p}(k)^{2}\left(1-a_{p}(k)^{-2} p^{k-2}\right)^{2} L^{*}\left(f_{k}^{\sharp} / K, \chi, k / 2\right) .
$$

Proof. The statement for $k=2$ follows from the fact that

$$
L_{p}\left(f_{\infty} / K, \Psi, 2\right)=\int_{L_{\Psi}^{\prime}} 1 d \mu_{L_{\Psi}}(x, y)=0 .
$$


Suppose now that $k$ belongs to $U \cap \mathbb{Z}^{>2}$. By eventually translating ( $\Psi, L_{\Psi}$ ) by an appropriate element of $R^{\times}$, we may assume that $\left|L_{\Psi}\right|=1$. Once this normalisation is made, Lemma 3.7 implies that

$$
\left\langle Q_{\Psi}(x, y)\right\rangle=Q_{\Psi}(x, y) \quad \text { on } L_{\Psi}^{\prime} .
$$

Definition 3.5 then implies that, for all $k \in U \cap \mathbb{Z}^{\geq 2}$,

$$
\mathcal{L}_{p}\left(f_{\infty} / K, \Psi, k\right)=\int_{L_{\Psi}^{\prime}} Q_{\Psi}(x, y)^{\frac{k-2}{2}} d \mu_{L_{\Psi}}(x, y) .
$$

Proposition 2.11 then yields

$$
\mathcal{L}_{p}\left(f_{\infty} / K, \Psi^{\sigma_{j}}, k\right)=\lambda_{B}(k) a_{p}(k)\left(1-a_{p}(k)^{-2} p^{k-2}\right) \cdot c_{k}^{\sharp}\left(L_{\Psi_{j}^{\sigma_{j}}}\right)\left(Q_{\Psi^{\sigma_{j}}}^{\frac{k-2}{2}}\right) .
$$

The result now follows from Proposition 3.3, in light of equation (90).

We need a similar interpolation formula in the case where $p$ is split in $K$. Assume for this rest of this section that $p$ satisfies this condition.

Fix an optimal embedding $(\Psi, L)$ and assume that $|L|=1$, after eventually translating $(\Psi, L)$ by an appropriate element of $R^{\times}$. Write $L=\mathbb{Z}_{p} v_{1} \oplus \mathbb{Z}_{p} v_{2}$, where $\left(v_{1}, v_{2}\right)$ is a $\mathbb{Z}_{p}$-basis of $L$ consisting of eigenvectors for the action of $\iota_{p} \Psi\left(K_{p}^{\times}\right)$. For each $j \in \mathbb{Z}$, define the lattice $L_{j}$ by

$$
L_{j}:=p^{j} \mathbb{Z}_{p} v_{1} \oplus \mathbb{Z}_{p} v_{2}
$$

Note that

$\left[\Psi, L_{j}\right] \in \operatorname{Emb}\left(\mathcal{O}_{K}, N^{+}, N^{-}\right), \quad\left[\Psi, L_{j}, L_{j+1}\right] \in \operatorname{Emb}\left(\mathcal{O}_{K}, p N^{+}, N^{-}\right)$, since the $L_{j}$ are preserved under the action of $\iota_{p} \Psi\left(\mathcal{O}_{K}\right)$.

Lemma 3.9. The value of $\mathcal{L}_{p}\left(f_{\infty} / K, \Psi, k\right)$ at $k=2$ is given by

$$
\mathcal{L}_{p}\left(f_{\infty} / K, \Psi, 2\right)=a_{p} \phi_{2}\left[\Psi, L_{-1}, L_{0}\right]-\phi_{2}\left[\Psi, L_{0}, L_{1}\right],
$$

while for $k \in U \cap \mathbb{Z}^{>2}$,

$$
\begin{aligned}
\mathcal{L}_{p}\left(f_{\infty} / K, \Psi, k\right)= & \lambda_{B}(k)\left(\left(a_{p}(k)+a_{p}(k)^{-1} p^{k-2}\right) \phi_{k}^{\sharp}\left[\Psi, L_{0}\right]\right. \\
& \left.-p^{\frac{k-2}{2}}\left(\phi_{k}^{\sharp}\left[\Psi, L_{-1}\right]+\phi_{k}^{\sharp}\left[\Psi, L_{1}\right]\right)\right) .
\end{aligned}
$$

Proof. Since

$$
L_{-1}^{\prime} \cap L_{0}^{\prime}=\mathbb{Z}_{p} v_{1} \times \mathbb{Z}_{p}^{\times} v_{2}, \quad \text { and } \quad L_{0}^{\prime} \cap L_{1}^{\prime}=p \mathbb{Z}_{p} v_{1} \times \mathbb{Z}_{p}^{\times} v_{2},
$$

we have

$$
L_{0}^{\prime \prime}=\left(L_{-1}^{\prime} \cap L_{0}^{\prime}\right)-\left(L_{0}^{\prime} \cap L_{1}^{\prime}\right)
$$


Therefore, since $\left\langle Q_{\Psi}\right\rangle^{k / 2-1}=Q_{\Psi}^{k / 2-1}$ on $L_{0}^{\prime \prime}$, we have

$$
\mathcal{L}_{p}\left(f_{\infty} / K, \Psi, k\right)=\int_{L_{-1}^{\prime} \cap L_{0}^{\prime}} Q_{\Psi}^{\frac{k-2}{2}} d \mu_{L_{0}}-\int_{L_{0}^{\prime} \cap L_{1}^{\prime}} Q_{\Psi}^{\frac{k-2}{2}} d \mu_{L_{0}} .
$$

By Lemma 2.9, this expression can be rewritten as

$$
\mathcal{L}_{p}\left(f_{\infty} / K, \Psi, k\right)=a_{p}(k) \int_{L_{-1}^{\prime} \cap L_{0}^{\prime}} Q_{\Psi}^{\frac{k-2}{2}} d \mu_{L_{-1}}-\int_{L_{0}^{\prime} \cap L_{1}^{\prime}} Q_{\Psi}^{\frac{k-2}{2}} d \mu_{L_{0}} .
$$

By Lemma 2.10,

$$
\mathcal{L}_{p}\left(f_{\infty} / K, \Psi, k\right)=\lambda_{B}(k)\left(a_{p}(k) c_{k}\left(L_{-1}, L_{0}\right)\left(Q_{\Psi}^{\frac{k-2}{2}}\right)-c_{k}\left(L_{0}, L_{1}\right)\left(Q_{\Psi}^{\frac{k-2}{2}}\right)\right) .
$$

This implies Lemma 3.9 for $k=2$, using (91). The case $k>2$ follows from (98), (90) and (58) by a direct calculation.

Choose a prime $\mathfrak{p}$ of $K$ above $p$, and let $\sigma_{\mathfrak{p}} \in G_{D}$ be its associated Frobenius element. Let $H_{0}$ be the subfield of $H$ which is fixed by $\sigma_{\mathfrak{p}}$, i.e., the maximal subfield of $H$ in which $\mathfrak{p}$ splits completely. Its Galois group over $K$ is identified with

$$
\Delta:=\hat{K}^{\times} / \hat{\mathcal{O}}^{\times} K^{\times}=G_{D} /\left\langle\sigma_{\mathfrak{p}}\right\rangle .
$$

We set $t=: \#\left\langle\sigma_{\mathfrak{p}}\right\rangle$, and $n:=\# \Delta$, so that $h=t n$.

Let $j_{p}: K \longrightarrow K_{\mathfrak{p}}=\mathbb{Q}_{p}$ denote the natural map of $K$ into its completion at $\mathfrak{p}$. The eigenbasis $\left(v_{1}, v_{2}\right)$ of $L$ for the action of $\iota_{p} \Psi\left(K_{p}\right)$ can be ordered in such a way that $\iota_{p} \Psi(\alpha)$ acts on $v_{1}$ by multiplication by $j_{p}(\alpha)$. With this convention, we have

\section{Lemma 3.10.}

$$
\sigma_{\mathfrak{p}} \cdot\left[\Psi, L_{j}\right]=\left[\Psi, L_{j+1}\right], \quad \sigma_{\mathfrak{p}} \cdot\left[\Psi, L_{j}, L_{j+1}\right]=\left[\Psi, L_{j+1}, L_{j+2}\right] .
$$

Proof. By (83),

$$
\sigma_{\mathfrak{p}} \cdot\left[\Psi, L_{j}\right]=\left[\Psi, \iota_{p} \hat{\Psi}\left(\gamma_{p}\right) L_{j}\right]
$$

where $\gamma_{p} \in \hat{K}^{\times}$is any idèle which is 1 at all places except $\mathfrak{p}$, where it is a uniformiser $\pi_{p}$ of $K_{\mathfrak{p}}^{\times}=\mathbb{Q}_{p}^{\times}$satisfying $\operatorname{ord}_{p}\left(\pi_{p}\right)=1$. The matrix $\iota_{p} \hat{\Psi}\left(\gamma_{p}\right)$ sends $v_{1}$ to $\pi_{p} v_{1}$, and multiplies $v_{2}$ by a scalar in $\mathbb{Z}_{p}^{\times}$. Hence

$$
\iota_{p} \hat{\Psi}\left(\gamma_{p}\right)\left(p^{j} \mathbb{Z}_{p} v_{1} \oplus \mathbb{Z}_{p} v_{2}\right)=\left(p^{j+1} \mathbb{Z}_{p} v_{1} \oplus \mathbb{Z}_{p} v_{2}\right) .
$$

The result follows. 
Write $\left[\Psi_{j}\right]=\left[\Psi, L_{j}\right]$. Recall that the genus character $\chi$ corresponds to a pair of Dirichlet characters $\chi_{1}$ and $\chi_{2}$, one of which is even and the other odd, and which are determined by the condition that for all rational primes $\ell=\lambda \bar{\lambda}$ which split in $K$,

$$
\chi(\lambda)=\chi(\bar{\lambda})=\chi_{1}(\ell)=\chi_{2}(\ell) .
$$

In particular, we have $\chi\left(\sigma_{\mathfrak{p}}\right)=\chi_{1}(p)=\chi_{2}(p)$.

Lemma 3.11. For all $[\Psi, L] \in \operatorname{Emb}\left(\mathcal{O}_{K}, N^{+}, N^{-}\right)$,

$$
\sum_{j=0}^{t-1} \chi\left(\sigma_{\mathfrak{p}}^{j}\right) \mathcal{L}_{p}\left(f_{\infty} / K, \Psi_{j}, 2\right)=a_{p}\left(1-a_{p}^{-1} \chi_{1}(p)\right) \sum_{j=0}^{t-1} \phi_{2}\left[\Psi, L_{j}, L_{j+1}\right],
$$

and for all $k \in U \cap \mathbb{Z}^{>2}$,

$$
\begin{aligned}
\sum_{j=0}^{t-1} \chi\left(\sigma_{\mathfrak{p}}^{j}\right) \mathcal{L}_{p}\left(f_{\infty} / K,\right. & \left.\Psi_{j}, k\right) \\
& =\lambda_{B}(k) a_{p}(k)\left(1-a_{p}(k)^{-1} \chi_{1}(p) p^{\frac{k-2}{2}}\right)^{2} \sum_{j=0}^{t-1} \phi_{k}^{\sharp}\left[\Psi_{j}\right] .
\end{aligned}
$$

Proof. Suppose that $k>2$. Lemma 3.10 implies that $\left[\Psi_{j}\right]=\left[\Psi_{j+t}\right]$, so that the function on $\mathbb{Z}$ which sends $j$ to $\phi_{k}^{\sharp}\left[\Psi_{j}\right]$ is periodic with period $t$. Lemma 3.11 is therefore a consequence of Lemma 3.9 by summing over the $\left[\Psi_{j}\right]$ with $0 \leq j \leq t-1$. The proof for $k=2$ is similar.

We now come to the analogue of Theorem 3.8 in the case where $p$ is split in $K$.

Theorem 3.12. Assume that $p$ is split in $K$. Then

$$
L_{p}\left(f_{\infty} / K, \chi, 2\right)=\left(1-\chi_{1}(p) a_{p}^{-1}\right)^{2}\left\langle\phi_{2}, \phi_{2}\right\rangle L^{*}(f / K, \chi, 1),
$$

and for any $k \in U \cap \mathbb{Z}^{>2}$,

$$
\begin{aligned}
& L_{p}\left(f_{\infty} / K, \chi, k\right) \\
& \qquad \lambda_{B}(k)^{2} a_{p}(k)^{2}\left(1-\chi_{1}(p) a_{p}(k)^{-1} p^{\frac{k-2}{2}}\right)^{4} L^{*}\left(f_{k}^{\sharp} / K, \chi, k / 2\right) \\
& =\lambda_{B}(k)^{2} a_{p}(k)^{2}\left(1-\chi_{1}(p) a_{p}(k)^{-1} p^{\frac{k-2}{2}}\right)^{2} L^{*}\left(f_{k} / K, \chi, k / 2\right) .
\end{aligned}
$$

Proof. We only give the details in the case $k>2$ since the case where $k=2$ is treated by a similar method but is a bit simpler. Let

$$
\mathcal{L}_{p}\left(f_{\infty} / K, \chi, k\right)=\sum_{j=1}^{h} \chi\left(\sigma_{j}\right) \mathcal{L}_{p}\left(f_{\infty} / K, \Psi^{\sigma_{j}}, k\right)
$$


denote the square root of the quantity to be evaluated in Theorem 3.12. It is convenient to rewrite the sum appearing in (99) by choosing lifts $\delta_{1}, \ldots, \delta_{n}$ of the $n$ distinct elements of $\Delta$ to the group $G_{D}$, and setting

$$
\left[\Psi^{(i)}, L^{(i)}\right]=\delta_{i} \cdot[\Psi, L] .
$$

After normalising these elements so that $\left|L^{(i)}\right|=1$, we define $\left[\Psi_{j}^{(i)}\right]=$ $\left[\Psi^{(i)}, L_{j}^{(i)}\right]$, with $L_{j}^{(i)}$ described as in (97) with $L$ replaced by $L^{(i)}$. By Lemma 3.10,

$$
\left[\Psi_{j}^{(i)}, L_{j}^{(i)}\right]=\delta_{i} \sigma_{\mathfrak{p}}^{j} \cdot[\Psi, L],
$$

and hence we may rewrite $\mathcal{L}_{p}\left(f_{\infty} / K, \chi, k\right)$ as a double sum:

$$
\mathcal{L}_{p}\left(f_{\infty} / K, \chi, k\right)=\sum_{i=1}^{n} \chi\left(\delta_{i}\right)\left(\sum_{j=0}^{t-1} \chi\left(\sigma_{\mathfrak{p}}^{j}\right) \mathcal{L}_{p}\left(f_{\infty} / K, \Psi_{j}^{(i)}, k\right)\right) .
$$

The inner sum can be evaluated using Lemma 3.11. This gives, after setting

$$
\begin{gathered}
\Theta:=\lambda_{B}(k) a_{p}(k)\left(1-a_{p}(k)^{-1} \chi_{1}(p) p^{\frac{k-2}{2}}\right)^{2}, \\
\mathcal{L}_{p}\left(f_{\infty} / K, \chi, k\right)=\Theta \times\left(\sum_{i=1}^{n} \chi\left(\delta_{i}\right) \sum_{j=0}^{t-1} \chi\left(\sigma_{\mathfrak{p}}^{j}\right) \phi_{k}^{\sharp}\left[\Psi_{j}^{(i)}\right]\right) \\
=\Theta \times\left(\sum_{j=1}^{h} \chi\left(\sigma_{j}\right) \phi_{k}^{\sharp}\left[\Psi^{\sigma_{j}}\right]\right) .
\end{gathered}
$$

The result follows from this last identity after invoking Proposition 3.3.

3.4. Calculation of derivatives. Assume throughout this section that $p$ is inert in $K$.

For any $[\Psi] \in \operatorname{Emb}\left(\mathcal{O}_{K}, N^{+}, N^{-}\right)$, we have $\mathcal{L}_{p}\left(f_{\infty} / K, \Psi ; 2\right)=0$ by (96). One is therefore led to consider the first derivative of this function at $k=2$. Given $\Psi=\left[\Psi, L_{\Psi}\right] \in \operatorname{Emb}\left(\mathcal{O}_{K}, N^{+}, N^{-}\right)$, use the indefinite integral of Definition 2.16 to write

$$
J_{\Psi}:=\int^{\tau_{\Psi}} \omega_{\phi}, \quad \bar{J}_{\Psi}:=\int^{\tau_{\Psi}^{\prime}} \omega_{\phi}
$$

The reader may check that the periods $J_{\Psi}$ and $\bar{J}_{\Psi}$ are well-defined and represent genuine invariants attached to elements of $\operatorname{Emb}\left(\mathcal{O}_{K}, N^{+}, N^{-}\right)$. Note that $J_{\Psi}$ belongs to $\mathbb{Q}_{p^{2}}$ and that $\bar{J}_{\Psi}$ is its conjugate under the action of $\operatorname{Gal}\left(\mathbb{Q}_{p^{2}} / \mathbb{Q}_{p}\right)$.

Proposition 3.13. For all $\Psi \in \operatorname{Emb}\left(\mathcal{O}_{K}, N^{+}, N^{-}\right)$,

$$
\frac{d}{d k} \mathcal{L}_{p}\left(f_{\infty} / K, \Psi ; k\right)_{k=2}=\frac{1}{2}\left(J_{\Psi}+\bar{J}_{\Psi}\right) \text {. }
$$


Proof. By definition of the $p$-adic $L$-function,

$$
\begin{aligned}
\frac{d}{d k} \mathcal{L}_{p}\left(f_{\infty} / K, \Psi ; k\right)_{k=2}= & \frac{1}{2} \int_{L_{\Psi}^{\prime}} \log \left(A\left(x-\tau_{\Psi} y\right)\left(x-\tau_{\Psi}^{\prime} y\right)\right) d \mu_{L_{\Psi}}(x, y) \\
=\frac{1}{2} & \left(\int_{L_{\Psi}^{\prime}} \log \left(x-\tau_{\Psi} y\right) d \mu_{L_{\Psi}}\right. \\
& \left.+\int_{L_{\Psi}^{\prime}} \log \left(x-\tau_{\Psi}^{\prime} y\right) d \mu_{L_{\Psi}}\right) .
\end{aligned}
$$

The claim follows from the definition of the indefinite integral and of $J_{\Psi}$.

The interest of Proposition 3.13 is revealed in Sect. 4, where the period $J_{\Psi}$ is equated with the formal group logarithm of certain Heegner points on $E(H)$, which are defined in Sect. 4.3.

Remark 3.14. Assume, as in Remark 3.6, that $h=1$ and consider the twovariable $p$-adic $L$-function $\mathscr{L}_{p}\left(f_{\infty} / K, k, s\right)$ defined in that remark. Given $k \in U \cap \mathbb{Z}^{\geq 2}$, the arguments of the classical $L$-function $L\left(f_{k}, s\right)$ that are critical in the sense of Deligne are the integers in the interval $[1, k-1]$. The restriction of $\mathcal{L}_{p}\left(f_{\infty} / K, k, s\right)$ to the boundary lines of this critical region admit particularly simple first derivatives:

$$
\begin{aligned}
& \bar{J}_{\Psi}=\frac{d}{d k} \mathcal{L}_{p}\left(f_{\infty} / K, k, 1\right)_{k=2}, \\
& J_{\Psi}=\frac{d}{d k} \mathcal{L}_{p}\left(f_{\infty} / K, k, k-1\right)_{k=2} .
\end{aligned}
$$

In the same vein, we note that

$$
\frac{d}{d s} \mathcal{L}_{p}\left(f_{\infty} / K, 2, s\right)_{s=1}=\int_{\tau_{\Psi}^{\prime}}^{\tau_{\Psi}} \omega_{\phi}=J_{\Psi}-\bar{J}_{\Psi} .
$$

This last result, expressing the leading term at $s=1$ of the "square root" of the anticyclotomic $p$-adic $L$-function of $E / K$ in terms of certain periods of $\omega_{\phi}$ attached to CM points, was the main result of [BD2].

We conclude with a formula for the first derivative of $\mathcal{L}_{p}\left(f_{\infty} / K, \chi, k\right)$ at $k=2$. Set

$$
J_{\chi}:=\sum_{\sigma \in G_{D}} \chi(\sigma) J_{\Psi^{\sigma}}
$$

and let $\bar{J}_{\chi}$ denote its conjugate over $\mathbb{Q}_{p^{2}}$.

Corollary 3.15. For all genus characters $\chi$ of $K$,

$$
\frac{d}{d k} \mathcal{L}_{p}\left(f_{\infty} / K, \chi, k\right)_{k=2}=\frac{1}{2}\left(J_{\chi}+\bar{J}_{\chi}\right) .
$$

Proof. This follows from Proposition 3.13. 


\section{Heegner points on Shimura curves}

The goal of this section is to prove that the period $J_{\Psi}$ appearing in Proposition 3.13 is the formal group logarithm of a Heegner point on $E$, arising from a parametrization of $E$ by a Shimura curve.

This is done in a sequence of steps. The first one reviews the theory of $p$-adic uniformisation of Shimura curves. Then, an explicit description of the modular parametrisation of $E$ by a Shimura curve is given in terms of the theory of $p$-adic integration. Using this theory, the periods $J_{\Psi}$ are interpreted as the formal logarithm of certain local points on E. Finally, the theory of complex multiplication is used to show that these local points are global points corresponding to moduli of certain abelian surfaces with complex multiplication.

4.1. Shimura curves. The weight two rigid analytic modular form $f_{\phi}(z)$ attached to $E$ in Sect. 2 defines a $\Gamma$-invariant differential $\omega_{\phi}$ on the $p$-adic upper half plane or, equivalently, a differential on the rigid analytic space $X_{\Gamma}:=\mathscr{H}_{p} / \Gamma$. Mumford's theory of $p$-adic uniformisation of nodal curves implies that $X_{\Gamma}$ is the rigid analytic space attached to the $\mathbb{C}_{p}$-points of a projective curve $X_{\mathbb{Q}_{p}}$ defined over $\mathbb{Q}_{p}$ : see for example [GvdP].

The Cerednik-Drinfeld theorem states that $X_{\mathbb{Q}_{p}}$ has a model over $\mathbb{Q}$. More precisely, let $\mathcal{B}$ be the indefinite quaternion algebra of discriminant $N^{-} p$ obtained from the quaternion algebra $B$ of Sect. 2 by interchanging the invariants $\infty$ and $p$. Write $\delta$ for a fixed Eichler order in $\mathcal{B}$ of level $N^{+}$. Fix an embedding

$$
\iota_{\infty}: \mathscr{B} \longrightarrow M_{2}(\mathbb{R}),
$$

and set

$$
\Gamma_{\infty}:=\iota_{\infty}\left(f_{1}^{\times}\right) \subset \mathbf{S L}_{2}(\mathbb{R}),
$$

where $\delta_{1}^{\times}$denotes the group of units in $\delta$ of reduced norm one. Let $\mathscr{H}_{\infty}$ denote the Poincaré upper half plane, and define the compact Riemann surface

$$
X_{\Gamma_{\infty}}:=\mathscr{H}_{\infty} / \Gamma_{\infty} .
$$

Shimura's work shows that $X_{\Gamma_{\infty}}$ is the complex analytic space attached to a projective curve $X$ defined over $\mathbb{Q}$. This is done by identifying $X_{\Gamma_{\infty}}$ with the complex points of the coarse moduli space for triples $(A, j, C)$, where:

(1) $A$ is an abelian scheme of relative dimension 2 over a $\mathbb{Q}$-scheme $U$;

(2) $j: \underline{s} \longrightarrow \operatorname{End}_{U}(A)$ is the action on $A$ of a fixed maximal order $\underline{\mathcal{s}}$ of $\mathscr{B}$ containing 8

(3) $C$ is a subgroup scheme of $A$, which is locally isomorphic to $\mathbb{Z} / N^{+} \mathbb{Z}$ and which is stable and locally cyclic for the action of $\&$ on $A$ induced by $j$. 
Using a variant of the above definition, it is possible to extend $X$ to a scheme over $\mathbb{Z}$.

Theorem 4.1 (Cerednik-Drinfeld). The space $X_{\Gamma}$ is the rigid analytic space attached to the $\mathbb{C}_{p}$-points of $X$. More precisely, there is an isomorphism defined over $\mathbb{Q}_{p^{2}}$ between the curves $X$ and $X_{\mathbb{Q}_{p}}$, where $\mathbb{Q}_{p^{2}}$ denotes the quadratic unramified extension of $\mathbb{Q}_{p}$.

Proof. See [BC].

Remark 4.2. The paper [BC] contains a detailed exposition of Drinfeld's proof of Theorem 4.1. This proof relies on an interpretation of the $p$-adic upper half plane $\mathcal{H}_{p}$ as the classifying space of certain formal groups of dimension 2 and height 4 , equipped with an action of the local quaternionic order $s_{p}:=\& \otimes \mathbb{Z}_{p}$. The identification of these formal groups with the formal groups at $p$ of the abelian surfaces parametrised by the Shimura curve $X$ yields a uniformisation of $X$ by $\mathscr{H}_{p}$.

The arithmetic structure carried by the groups $\Gamma$, resp. $\Gamma_{\infty}$ gives rise to a family of Hecke correspondences $T_{n}^{(p)}$, resp. $T_{n}^{(\infty)}$ on $X_{\Gamma}$, resp. $X_{\Gamma_{\infty}}$, indexed by the positive integers. The correspondences $T_{n}^{(p)}$ and $T_{n}^{(\infty)}$ induce the same correspondence on $X$, denoted by $T_{n}$. The operator $T_{n}$ is defined over $\mathbb{Q}$.

\subsection{Modular parametrizations. Let}

$$
J:=\operatorname{Div}^{0}(X) / P(X)
$$

denote the Jacobian of the Shimura curve $X$ introduced in the previous section, whose points over an extension $F$ of $\mathbb{Q}$ correspond to $F$-rational classes of degree zero divisors on $X$ modulo the subgroup $P(X)$ of principal divisors. The Jacquet-Langlands correspondence combined with a version of the Eichler-Shimura theory for the Shimura curve $X$ shows that $J$ has a simple factor $A_{\phi}$ satisfying

$$
L\left(A_{\phi}, s\right)=L(E, s) .
$$

By the isogeny theorem, the elliptic curves $A_{\phi}$ and $E$ are isogenous over $\mathbb{Q}$. Hence there exists a non-constant morphism

$$
\varphi_{E}: J \longrightarrow E
$$

defined over $\mathbb{Q}$, which is called a modular parametrisation attached to $X$ and $E$. (One might want to normalise $\varphi_{E}$ by requiring, for example, that it not factor through any non-trivial endomorphism of $E$, but this is not really necessary.)

Let $\operatorname{Div}^{0}\left(X_{\Gamma}\right)$ denote the groups of degree zero divisors supported on $X_{\Gamma}:=\mathscr{H}_{p} / \Gamma$, and let

$$
J_{\Gamma}:=\operatorname{Div}^{0}\left(X_{\Gamma}\right) / P\left(X_{\Gamma}\right)
$$


Theorem 4.1 identifies $J\left(\mathbb{C}_{p}\right)$ with $J_{\Gamma}$. We wish to give an explicit $p$-adic analytic description of $\varphi_{E}$ in terms of this identification.

Let $\omega_{E}$ be a Néron differential on $E$, and let $\varphi_{E}^{*}\left(\omega_{E}\right)$ denote its pullback to $J$. This differential is proportional to $\omega_{\phi}$. After possible replacing $\omega_{\phi}$ by a non-zero rational multiple, we may assume that

$$
\omega_{\phi}=\varphi_{E}^{*}\left(\omega_{E}\right) .
$$

The $p$-adic lattice $Q_{\phi}$ defined in Theorem 2.24 is then contained in the Tate lattice of $E$, so that the function from $\operatorname{Div}^{0}\left(\mathscr{H}_{p} / \Gamma\right)$ to $E\left(\mathbb{C}_{p}\right)$ defined by

$$
d \mapsto \Phi_{\text {Tate }}\left(f_{d} \omega_{\phi}\right)
$$

is well-defined. The next result is proved in [GvdP].

Theorem 4.3. The modular parametrisation $\varphi_{E}: \operatorname{Div}^{0}(X) \longrightarrow E$ can be described over $\mathbb{Q}_{p^{2}}$ by the formula

$$
\varphi_{E}(d)=\Phi_{\text {Tate }}\left(\oint_{d} \omega_{\phi}\right) .
$$

4.3. Heegner points. Recall that $H$ denotes the Hilbert class field of $K$, whose Galois group over $K$ is identified by class field theory with the class group $G_{D}$ of $K$. Since $p$ is inert in $K$, it splits completely in the extension $H / K$. Fix a prime $\mathfrak{p}$ of $H$ above $p$; this determines an embedding of $H$ into $\mathbb{Q}_{p^{2}}$ and we can thus view $H$ as a subfield of this $p$-adic field.

Let $[\Psi]=[\Psi, b]=[\Psi, L] \in \operatorname{Emb}\left(\mathcal{O}_{K}, N^{+}, N^{-}\right)$be a class of optimal embeddings, normalised so that $b$ belongs to $\hat{R}^{\times}$and so that $L$ is an even lattice in $\mathbb{Q}_{p}^{2}$. Let $\tau_{\Psi} \in \mathcal{H}_{p}^{\prime}$ denote the fixed point of $\iota_{p} \Psi\left(K_{p}^{\times}\right)$on $\mathscr{H}_{p}$ normalised as in (85). Since the embedding $\Psi$ is determined by $[\Psi]$ up to conjugation by $\Gamma$, the image of $\tau_{\Psi}$ in $\mathscr{H}_{p} / \Gamma$ depends only on $[\Psi]$. Let $\tilde{P}_{\Psi} \in X_{\Gamma}\left(\mathbb{Q}_{p^{2}}\right)=X\left(\mathbb{Q}_{p^{2}}\right)$ be the point on $X$ associated to $\tau_{\Psi}$.

The next result is the main theorem of complex multiplication.

Proposition 4.4. For all $[\Psi] \in \operatorname{Emb}\left(\mathcal{O}_{K}, N^{+}, N^{-}\right)$, the point $\tilde{P}_{\Psi}$ belongs to $X(H)$, and for all $\sigma \in G_{D}$,

$$
\tilde{P}_{\Psi^{\sigma}}=\sigma\left(\tilde{P}_{\Psi}\right) .
$$

Sketch. The proof of this result rests on Drinfeld's theory of $p$-adic uniformisation of Shimura curves alluded to in Remark 4.2. This theory shows that the image of $\tau_{\Psi}$ in $\mathscr{H}_{p} / \Gamma$ corresponds to an abelian surface with quaternionic multiplication, having complex multiplication by the ring of integers of $K$. Such a surface is isogenous to a product of elliptic curves with complex multiplication by $\mathcal{O}_{K}$. The claim then follows from the theory of complex multiplication for abelian varieties. See Theorem 5.3 of [BD2] for more details. 
Given a quadratic character $\chi$ of $G_{D}$, let $H_{\chi}=\mathbb{Q}\left(\sqrt{D_{1}}, \sqrt{D_{2}}\right)$ denote as before the biquadratic field cut out by $\chi$ (or the quadratic field $K$, if $\chi$ is the trivial character). Define a Heegner divisor of degree 0 attached to $\chi$ by choosing an auxiliary prime $\ell \not N$ if $\chi=1$ and setting

$$
\tilde{P}_{\chi}= \begin{cases}\left(\ell+1-a_{\ell}\right)^{-1}\left(\ell+1-T_{\ell}\right)\left(\sum_{\sigma \in G_{D}} \tilde{P}_{\Psi^{\sigma}}\right) & \text { if } \chi=1, \\ \sum_{\sigma \in G_{D}} \chi(\sigma) \tilde{P}_{\Psi^{\sigma}} & \text { if } \chi \neq 1 .\end{cases}
$$

By Proposition 4.4, the element $\tilde{P}_{\chi}$ belongs to $\operatorname{Div}^{0}(X)\left(H_{\chi}\right)^{\chi}$ if $\chi \neq 1$, and to $\operatorname{Div}^{0}(X)(K) \otimes \mathbb{Q}$ if $\chi$ is the trivial character. Furthermore, the Galois group of $H_{\chi}$ over $K$ acts on $\tilde{P}_{\chi}$ via the character $\chi$.

We define

$$
P_{\chi}:=\varphi_{E}\left(\tilde{P}_{\chi}\right) \in \begin{cases}E\left(H_{\chi}\right)^{\chi} & \text { if } \chi \neq 1 \\ E(K) \otimes \mathbb{Q} & \text { if } \chi=1 .\end{cases}
$$

The global point $P_{\chi}$ is called the Heegner point attached to $\Psi$ and $\chi$.

Recall the formal group logarithm $\log _{E}$ defined in (7) in the Introduction, and let frob ${ }_{p}$ denote the Frobenius element attached to the rational prime $p$ in the abelian extension $H_{\chi} / \mathbb{Q}$.

Proposition 4.5. For all genus characters $\chi$ of $G_{D}$,

$$
J_{\chi}=\log _{E}\left(P_{\chi}\right), \quad \bar{J}_{\chi}=a_{p} \log _{E}\left(\operatorname{frob}_{p} P_{\chi}\right) .
$$

Proof. If $\chi \neq 1$, then $\tilde{P}_{\chi}$ is a degree zero divisor on $X$ and therefore by Theorem 4.3,

$$
P_{\chi}=\Phi_{\text {Tate }}\left(f_{\tilde{P}_{\chi}} \omega_{\phi}\right) .
$$

Applying $\log _{E}$ to both sides gives

$$
\log _{E}\left(P_{\chi}\right)=\int_{\tilde{P}_{\chi}} \omega_{\phi}
$$

But this last expression is equal to $J_{\chi}$, by Corollary 2.26 and the definition of the period $J_{\chi}$ in terms of the indefinite integral. The proof for $\chi=1$ follows in a similar way, using the compatibility of the indefinite integral with Hecke operators asserted in Proposition 2.18. The formula for $\bar{J}_{\chi}$ is obtained by applying the generator $s_{p}$ of $\operatorname{Gal}\left(\mathbb{Q}_{p^{2}} / \mathbb{Q}_{p}\right)$ to both sides, and noting that $\Phi_{\text {Tate }}$ and $\log _{E}$ do not commute with this element when $E$ has non-split multiplicative reduction at $p$, and that, more precisely, for any $P \in E\left(\mathbb{Q}_{p^{2}}\right)$,

$$
s_{p}\left(\log _{E}(P)\right)=a_{p} \log _{E}\left(\operatorname{frob}_{p} P\right) .
$$


We note the following facts about the Heegner point $P_{\chi}$.

Theorem 4.6. The point $P_{\chi}$ is of infinite order if and only if $L^{\prime}(E / K, \chi, 1)$ $\neq 0$. In that case, the rank of $E\left(H_{\chi}\right)^{\chi}$ is equal to one.

Proof. The first statement follows from the work of Zhang [Zh2] extending the formula of Gross and Zagier to Shimura curve parametrisations (attached to a quaternion algebra over a totally real field $F$, although only the case $F=\mathbb{Q}$ is needed here.) The second statement follows from the methods of Kolyvagin and Logachev [KL] (cf. Theorem A of [Zh1]).

For each integer $m \mid N$ satisfying $\operatorname{gcd}(m, N / m)=1$, let $w_{m} \in\{-1,1\}$ denote the eigenvalue of the Fricke involution $W_{m}$ acting on $f$. The key facts about $w_{m}$ that will be used are:

(1) The sign in the functional equation for $L(f, s)$ is equal to $-w_{N}$. More generally, if $\chi_{1}$ is a quadratic Dirichlet character of conductor prime to $N$, the sign in the functional equation for $L\left(f, \chi_{1}, s\right)$ is $-w_{N} \chi_{1}(-N)$.

(2) The element $w_{p}$ is equal to $-a_{p}$. In particular, $L_{p}\left(f, \chi_{1}, s\right)$ has an exceptional zero at $s=1$ if and only if $\chi_{1}(p)=-w_{p}$, i.e., $\chi_{1}(p)=a_{p}$. Since

Let $\chi_{1}$ and $\chi_{2}$ denote the quadratic Dirichlet characters associated to $\chi$.

$$
\chi_{1}(-N) \chi_{2}(-N)=\epsilon_{K}(-N)=-1,
$$

we may order $\left(\chi_{1}, \chi_{2}\right)$ in such a way that

$$
\chi_{1}(-N)=w_{N}, \quad \chi_{2}(-N)=-w_{N} .
$$

With this fixed ordering, we have the following result which gives a more precise information about the location of the Heegner point $P_{\chi}$.

Theorem 4.7. The image of $P_{\chi}$ in $E\left(H_{\chi}\right) \otimes \mathbb{Q}$ belongs to $\left(E\left(\mathbb{Q}\left(\sqrt{D_{1}}\right)\right)\right.$ $\otimes \mathbb{Q})^{x_{1}}$.

Proof. If $P_{\chi}$ is of finite order, its image in $E\left(H_{\chi}\right) \otimes \mathbb{Q}$ is trivial and there is nothing to prove. Otherwise, Theorem 4.6 asserts that

$$
L(E / K, \chi, s)=L\left(E, \chi_{1}, s\right) L\left(E, \chi_{2}, s\right)
$$

vanishes to order 1 at $s=1$. By the chosen ordering of the characters $\chi_{1}$ and $\chi_{2}$, the function $L\left(E, \chi_{j}, s\right)$ vanishes to odd order when $j=1$, and to even order when $j=2$. Hence the factors on the right in (101) vanish to order 1 and 0 respectively. Kolyvagin's theorem implies that

$$
\operatorname{dim}_{\mathbb{Q}}\left(E\left(\mathbb{Q}\left(\sqrt{D_{1}}\right)\right) \otimes \mathbb{Q}\right)^{\chi_{1}}=1 ; \quad \operatorname{dim}_{\mathbb{Q}}\left(E\left(\mathbb{Q}\left(\sqrt{D_{2}}\right)\right) \otimes \mathbb{Q}\right)^{\chi_{2}}=0 .
$$

The result follows. 
Corollary 4.8. $\operatorname{frob}_{p}\left(P_{\chi}\right)=\chi_{1}(p) P_{\chi}$.

Proof. This follows directly from Theorem 4.7.

4.4. Heegner points and derivatives of Hida $L$-functions. Assume as in the previous section that the pair $\left(\chi_{1}, \chi_{2}\right)$ of Dirichlet characters associated to the genus character $\chi$ of $K$ has been ordered in such a way that $\chi_{1}(-N)=w_{N}$.

\section{Theorem 4.9.}

$$
\frac{d}{d k} \mathcal{L}_{p}\left(f_{\infty} / K, \chi, k\right)_{k=2}=\frac{1}{2}\left(1+\chi_{1}(p) a_{p}\right) \log _{E}\left(P_{\chi}\right) .
$$

Proof. Applying Corollary 3.15 followed by Proposition 4.5,

$$
\begin{aligned}
\frac{d}{d k} \mathcal{L}_{p}\left(f_{\infty} / K, \chi, k\right)_{k=2} & =\frac{1}{2}\left(J_{\chi}+\bar{J}_{\chi}\right) \\
& =\frac{1}{2}\left(\log _{E}\left(P_{\chi}\right)+a_{p} \log _{E}\left(\operatorname{frob}_{p} P_{\chi}\right)\right) .
\end{aligned}
$$

The theorem now follows from Corollary 4.8.

\section{Corollary 4.10.}

$$
\frac{d^{2}}{d k^{2}} L_{p}\left(f_{\infty} / K, \chi ; k\right)_{k=2}= \begin{cases}2 \log _{E}\left(P_{\chi}\right)^{2} & \text { if } \chi_{1}(p)=a_{p} \\ 0 & \text { if } \chi_{1}(p)=-a_{p} .\end{cases}
$$

\section{The main result}

5.1. A factorisation of $p$-adic $L$-functions. Define the function

$$
\eta(k):= \begin{cases}\left\langle\phi_{2}, \phi_{2}\right\rangle & \text { if } k=2 ; \\ a_{p}(k)^{2} D^{k / 2-1} \frac{\lambda_{B}(k)^{2}}{\lambda^{+}(k) \lambda^{-}(k)}, & \text { for } k \in U \cap \mathbb{Z}^{>2} .\end{cases}
$$

Note that the scalar $\eta(k) \in \mathbb{C}_{p}$ depends only on $k$, and on the factorisation (32), not on $K$.

Proposition 5.1. For all $k \in U \cap \mathbb{Z}^{\geq 2}$, and all genus characters $\chi$,

$$
L_{p}\left(f_{\infty} / K, \chi ; k\right)=\eta(k) L_{p}\left(f_{\infty}, \chi_{1}, k, k / 2\right) L_{p}\left(f_{\infty}, \chi_{2}, k, k / 2\right) .
$$

Proof. Assume first that $k>2$. The following formula follows from comparing the Euler products on both sides:

$$
L\left(f_{k}^{\sharp} / K, \chi, k / 2\right)=L\left(f_{k}^{\sharp}, \chi_{1}, k / 2\right) L\left(f_{k}^{\sharp}, \chi_{2}, k / 2\right) .
$$


By definition (cf. Proposition 1.3)

$$
\begin{aligned}
& L^{*}\left(f_{k}^{\sharp}, \chi_{1}, k / 2\right):=\frac{(k / 2-1) ! \tau\left(\chi_{1}\right)}{(-2 \pi i)^{k / 2-1} \Omega_{k}^{ \pm}} L\left(f_{k}^{\sharp}, \chi_{1}, k / 2\right) \\
& L^{*}\left(f_{k}^{\sharp}, \chi_{2}, k / 2\right):=\frac{(k / 2-1) ! \tau\left(\chi_{2}\right)}{(-2 \pi i)^{k / 2-1} \Omega_{k}^{\mp}} L\left(f_{k}^{\sharp}, \chi_{2}, k / 2\right) .
\end{aligned}
$$

The choice of complex periods that was made in (14) implies that

$$
\Omega_{k}^{ \pm} \Omega_{k}^{\mp}=\left\langle f_{k}^{\sharp}, f_{k}^{\sharp}\right\rangle .
$$

Hence by referring to (93), we get

$$
L^{*}\left(f_{k}^{\sharp} / K, \chi, k / 2\right)=D^{k / 2-1} L^{*}\left(f_{k}^{\sharp}, \chi_{1}, k / 2\right) L^{*}\left(f_{k}^{\sharp}, \chi_{2}, k / 2\right) .
$$

By comparing the Euler factors that appear in (31) following Theorem 1.12 (with $\chi=\chi_{1}$ and $\chi=\chi_{2}$ ) with the Euler factor that occurs in Theorem 3.12 (if $p$ is split in $K$ ) and Theorem 3.8 (if $p$ is inert in $K$ ), we find

$$
L_{p}\left(f_{\infty} / K, \chi ; k\right)=\eta(k) L_{p}\left(f_{\infty}, \chi_{1}, k, k / 2\right) L_{p}\left(f_{\infty}, \chi_{2}, k, k / 2\right),
$$

as was to be shown. The proof for $k=2$ is identical, and is therefore omitted.

Although Hida theory gives little control on the variation with $k$ of the quantities $\lambda^{+}(k), \lambda^{-}(k)$, and $\lambda_{B}(k)$ taken individually, it is still possible to prove the following result.

Proposition 5.2. The function $\eta(k)$ extends to a p-adic analytic function of $k \in U$.

Proof. Consider the set of primitive quadratic Dirichlet characters $\chi_{1}$ of conductor prime to $N$, satisfying

$$
\chi_{1}(-N)=-w_{N} ; \quad \chi_{1}(p)=-a_{p} .
$$

The condition on $\chi_{1}(-N)$ implies that $L\left(f, \chi_{1}, s\right)$ vanishes to even order at $s=1$. By the main result of [MM], there are infinitely many such $\chi_{1}$ satisfying

$$
L\left(f, \chi_{1}, 1\right) \neq 0 .
$$

The second condition in (104) imposed on $\chi_{1}$ implies that $L_{p}\left(f, \chi_{1}, s\right)$ does not have an exceptional zero, so that

$$
L_{p}\left(f, \chi_{1}, 1\right) \text { belongs to } \mathbb{Q}^{\times} .
$$

Fix one character $\chi_{1}$ satisfying (104) as well as the non-vanishing condition (105). Consider now the set of Dirichlet characters $\chi_{2}$ of conductor prime to $N$ and to the conductor of $\chi_{1}$, satisfying 
(1) $\chi_{2}(\ell)=\chi_{1}(\ell)$, for all $\ell \mid p N^{+}$;

(2) $\chi_{2}(\ell)=-\chi_{1}(\ell)$, for all $\ell \mid N^{-}$and $\ell=-1$;

Note that, since $N^{-}$has an odd number of prime factors, $\chi_{2}(-N)=$ $\chi_{1}(-N)$, so that the $L$-series $L\left(f, \chi_{2}, s\right)$ also vanishes to even order at $s=1$. Invoking the non-vanishing result of $[\mathrm{MM}]$ one more time, and noting that the restriction placed on $\chi_{2}(p)$ prevents $L_{p}\left(f, \chi_{2}, s\right)$ from acquiring an extra zero at $s=1$, one obtains a character $\chi_{2}$ such that

$$
L\left(f, \chi_{2}, 1\right) \neq 0, \quad L_{p}\left(f, \chi_{2}, 1\right) \neq 0 .
$$

It follows from these choices of $\chi_{1}$ and $\chi_{2}$ that the functions

$$
L_{p}\left(f_{\infty} / K, \chi, k\right), \quad L_{p}\left(f_{\infty}, \chi_{1}, k, k / 2\right), \quad \text { and } \quad L_{p}\left(f_{\infty}, \chi_{2}, k, k / 2\right)
$$

are analytic functions on $U$ that do not vanish at $k=2$. The result now follows from Proposition 5.1.

In particular, we have proved:

Corollary 5.3. For all $k \in U$,

$$
L_{p}\left(f_{\infty} / K, \chi, k\right)=\eta(k) L_{p}\left(f_{\infty}, \chi_{1}, k, k / 2\right) L_{p}\left(f_{\infty}, \chi_{2}, k, k / 2\right),
$$

where $\eta(k)$ is a p-adic analytic function of $k$ satisfying $\eta(2)=\left\langle\phi_{2}, \phi_{2}\right\rangle$.

5.2. Proof of the main result. We can now state and prove the main result of this paper, which implies Theorem 1 of the Introduction when specialised to the trivial character.

Theorem 5.4. Suppose that $E$ has at least two distinct primes of semistable reduction. Let $\chi_{1}$ be a Dirichlet character of conductor prime to $N$, satisfying $\chi_{1}(-N)=w_{N}$, and $\chi_{1}(p)=a_{p}$. Then

(1) The $p$-adic L-function $L_{p}\left(f_{\infty}, \chi_{1}, k, k / 2\right)$ vanishes to order at least 2 at $k=2$.

(2) There exists a global point $\mathbf{P}_{\chi_{1}} \in\left(E\left(\mathbb{Q}\left(\sqrt{D_{1}}\right)\right) \otimes \mathbb{Q}\right)^{\chi_{1}}$, and $\ell \in \mathbb{Q}^{\times}$, such that

$$
\frac{d^{2}}{d k^{2}} L_{p}\left(f_{\infty}, \chi_{1}, k, k / 2\right)_{k=2}=\ell \log ^{2}\left(\mathbf{P}_{\chi_{1}}\right)
$$

(3) The point $\mathbf{P}_{\chi_{1}}$ is of infinite order if and only if $L^{\prime}\left(E / \mathbb{Q}, \chi_{1}, 1\right) \neq 0$.

(4) The image of $\ell$ in $\mathbb{Q}^{\times} /\left(\mathbb{Q}^{\times}\right)^{2}$ is equal to that of $L^{*}(f, \psi, 1)$, where $\psi$ is any quadratic Dirichlet character satisfying

(a) $\psi(\ell)=\chi_{1}(\ell)$ for all primes $\ell$ dividing $M=N / p$;

(b) $\psi(p)=-\chi_{1}(p)$;

(c) $L(f, \psi, 1) \neq 0$. 
Proof. 1. Since $\chi_{1}(-N)=w_{N}$, the classical $L$-function $L\left(f, \chi_{1}, s\right)$ has sign -1 in its functional equation, and in particular vanishes at $s=1$. Furthermore, the condition $\chi_{1}(p)=a_{p}$ implies that $L_{p}\left(f, \chi_{1}, s\right)$ has an exceptional zero at $s=1$, so that the sign in the functional equation in this $p$-adic $L$-function is 1 . In particular, the order of vanishing of $L_{p}\left(f, \chi_{1}, s\right)$ at $s=1$ is at least 2 :

$$
\operatorname{ord}_{s=1} L_{p}\left(f_{\infty}, \chi_{1}, 2, s\right) \geq 2 .
$$

On the other hand, formula (30) expresses $L_{p}\left(f_{\infty}, \chi_{1}, k, 1\right)$ as a product of two analytic functions of $k$ which both vanish at $k=2$ :

$$
L_{p}\left(f, \chi_{1}, k, 1\right)=\left(1-a_{p}(k)^{-1} \chi_{1}(p)\right) L_{p}^{*}\left(f_{\infty}, \chi_{1}, k\right) .
$$

It follows that

$$
\operatorname{ord}_{k=2} L_{p}\left(f_{\infty}, \chi_{1}, k, 1\right) \geq 2 .
$$

Equations (107) and (108) together imply that $L_{p}\left(f_{\infty}, \chi_{1}, k, s\right)$ vanishes to order at least 2 at $(k, s)=(2,1)$, and Part 1 of Theorem 5.4 follows.

2. Choose an auxiliary quadratic Dirichlet character $\chi_{2}$ of conductor prime to the conductor of $\chi_{1}$, and satisfying the following conditions, by invoking the non-vanishing result of $[\mathrm{MM}]$, much as in the proof of Proposition 5.2.

(1) $\chi_{2}(\ell)=\chi_{1}(\ell)$, for all $\ell \mid N^{+}$;

(2) $\chi_{2}(\ell)=-\chi_{1}(\ell)$, for all $\ell \mid p N^{-}$or $\ell=-1$;

(3) $L\left(f, \chi_{2}, 1\right) \neq 0$.

The conditions placed on $\chi_{2}$ imply that the quadratic field $K$ attached to the character $\epsilon_{K}:=\chi_{1} \chi_{2}$ is admissible relative to $N=p N^{+} N^{-}$, and that $p$ is inert in $K$. In particular, the $p$-adic $L$-function $L_{p}\left(f, \chi_{2}, s\right)$ does not have an exceptional zero at $s=1$, so that $L_{p}\left(f_{\infty}, \chi_{2}, 2,1\right)$ belongs to $\mathbb{Q}^{\times}$.

Let $\chi$ be the genus character of $K$ associated to the pair of quadratic characters $\chi_{1}$ and $\chi_{2}$. By Corollary 5.3, we have

$$
L_{p}\left(f_{\infty} / K, \chi, k\right)=\eta(k) L_{p}\left(f_{\infty}, \chi_{1}, k, k / 2\right) L_{p}\left(f_{\infty}, \chi_{2}, k, k / 2\right) .
$$

Taking second derivatives at $k=2$, and applying Corollary 4.10, we find

$$
2 \log _{E}\left(P_{\chi}\right)^{2}=\eta(2) \frac{d^{2}}{d k^{2}} L_{p}\left(f_{\infty}, \chi_{1}, k, k / 2\right)_{k=2} L_{p}\left(f, \chi_{2}, 1\right) .
$$

It follows that

$$
\frac{d^{2}}{d k^{2}} L_{p}\left(f_{\infty}, \chi_{1}, k, k / 2\right)_{k=2}=\ell \log _{E, p}\left(P_{\chi}\right)^{2} .
$$

where

$$
\ell^{-1}=\eta(2) L^{*}\left(f, \chi_{2}, 1\right)=\left\langle\phi_{2}, \phi_{2}\right\rangle L^{*}\left(f, \chi_{2}, 1\right) .
$$

This proves Part 2, setting $\mathbf{P}_{\chi_{1}}:=P_{\chi}$. 
3. To show Part 3, recall that Theorem 4.6 states that the Heegner point $\mathbf{P}_{\chi_{1}}$ is of infinite order if and only if the derivative of the classical $L$-series

$$
L^{\prime}(E / K, \chi, 1)=L^{\prime}\left(E, \chi_{1}, 1\right) L\left(E, \chi_{2}, 1\right)
$$

is non-zero. Since the second factor in the expression on the right is nonvanishing by the choice of $\chi_{2}$, the result follows.

4. Finally, (109) shows that

$$
\ell=\left\langle\phi_{2}, \phi_{2}\right\rangle L^{*}\left(f, \chi_{2}, 1\right) \quad\left(\bmod \left(\mathbb{Q}^{\times}\right)^{2}\right) .
$$

Choose a character $\psi$ satisfying the three conditions of Part 4 of Theorem 1, and having conductor prime to that of $\chi_{2}$. Then the quadratic field $\tilde{K}$ cut out by the character $\epsilon=\psi \chi_{2}$ is admissible relative to the factorisation $p N^{+} N^{-}$, and $p$ is split in $\tilde{K}$. Let $\tilde{\chi}$ be the genus character attached to $\left(\psi, \chi_{2}\right)$. Then it follows from Proposition 3.4 that

$$
\left\langle\phi_{2}, \phi_{2}\right\rangle=L^{*}(f / \tilde{K}, \tilde{\chi}, 1)=L^{*}(f, \psi, 1) L^{*}\left(f, \chi_{2}, 1\right) \quad\left(\bmod \left(\mathbb{Q}^{\times}\right)^{2}\right) .
$$

The result follows by combining (110) and (111).

\section{References}

[BC] Boutot, J.-F., Carayol, H.: Uniformisation $p$-adique des courbes de Shimura: les théorèmes de Cerednik et de Drinfeld. Courbes modulaires et courbes de Shimura (Orsay, 1987/1988). Astérisque 196-197(7), 45-158 (1991/1992)

[BD1] Bertolini, M., Darmon, H.: Heegner points on Mumford-Tate curves. Invent. Math. 126, 413-456 (1996)

[BD2] Bertolini, M., Darmon, H.: Heegner points, $p$-adic $L$-functions and the Cerednik-Drinfeld uniformization. Invent. Math. 131(3), 453-491 (1998)

[BD3] Bertolini, M., Darmon, H.: The rationality of Stark-Heegner points over genus fields of real quadratic fields. To appear in Ann. Math.

[BDG] Bertolini, M., Darmon, H., Green, P.: Periods and points attached to quadratic algebras. MSRI Publ. 49, pp. 323-367. Cambridge University Press 2004

[BDIS] Bertolini, M., Darmon, H., Iovita, A., Spiess, M.: Teitelbaum's exceptional zero conjecture in the anticyclotomic setting. Am. J. Math. 124, 411-449 (2002)

[BDI] Bertolini, M., Darmon, H., Iovita, A.: Families of automorphic forms on definite quaternion algebras and Teitelbaum's conjecture. Submitted

$[\mathrm{Bu}] \quad$ Buzzard, K.: On $p$-adic families of automorphic forms. Modular curves and abelian varieties. Prog. Math. vol. 224, pp. 23-44. Birkhäuser, Basel (2004)

[Ch] Chenevier, G.: Une correspondance de Jacquet-Langlands $p$-adique. Duke Math. J. 126(1), 161-194 (2005)

[Da] Darmon, H.: Rational points on modular elliptic curves. CBMS Regional Conference Series in Mathematics, 101. Published for the Conference Board of the Mathematical Sciences, Washington, DC. Providence, RI: Am. Math. Soc. 2004

[Gre] Greenberg, R.: Elliptic curves and p-adic deformations. Elliptic curves and related topics. CRM Proc. Lect. Notes vol. 4, pp. 101-110. Providence, RI: Am. Math. Soc. 1994 
[Gr] Gross, B.H.: Heights and the special values of $L$-series. Number theory (Montreal, Que., 1985). CMS Conf. Proc. vol. 7, pp. 115-187. Providence, RI: Am. Math. Soc. 1987

[GS] Greenberg, R., Stevens, G.: $p$-adic $L$-functions and $p$-adic periods of modular forms. Invent. Math. 111(2), 407-447 (1993)

[GvdP] Gerritzen, L., van der Put, M.: Shottky Groups and Mumford Curves. Lect. Notes Math., vol. 817. Berlin: Springer 1980

[Ha1] Hatcher, R.: Heights and $L$-series. Canad. J. Math. 42(3), 533-560 (1990)

[Ha2] Hatcher, R.: Special values of $L$-series. Proc. Am. Math. Soc. 114(2), 337-343 (1992)

[HX1] Hui, X.: Central values of Rankin $L$-series. To appear in Int. Math. Res. Not.

[HX2] Hui, X.: Central values of $L$-functions over CM fields. To appear in J. Number Theory

[JL] Jacquet, H., Langlands, R.P.: Automorphic forms on GL(2). Lect. Notes Math., vol. 114. Springer, Berlin New York (1970)

[KL] Kolyvagin, V.A., Logachev, D.Y.: Finiteness of $\amalg$ over totally real fields. Izv. Akad. Nauk SSSR Ser. Mat. 55(4), 851-876 (1991); translation in Math. USSR-Izv. 39(1), 829-853 (1992)

[Man] Manin, J.I.: Parabolic Points and Zeta Functions of Modular Curves. Izv. Akad. Nauk SSSR Ser. Mat. 36(1), 19-66 (1972)

[MM] Murty, M.R., Murty, K.V.: Non-vanishing of $L$-functions and applications. Prog. Math., vol. 157. Birkhäuser, Basel (1997)

[M-Sw-D] Mazur, B., Swinnerton-Dyer, P.: Arithmetic of Weil curves. Invent. Math. 25, 1-61 (1974)

[MTT] Mazur, B., Tate, J., Teitelbaum, J.: On $p$-adic analogues of the conjectures of Birch and Swinnerton-Dyer. Invent. Math. 84(1), 1-48 (1986)

[PR] Perrin-Riou, B.: Fonctions $L p$-adiques d'une courbe elliptique et points rationnels. Ann. Inst. Fourier 43(4), 945-995 (1993)

[Ru] Rubin, K.: $p$-adic $L$-functions and rational points on elliptic curves with complex multiplication. Invent. Math. 107(2), 323-350 (1992)

[Se] Serre, J.P.: Trees. Springer, Berlin (1980)

[Te] Teitelbaum, J.T.: Values of $p$-adic $L$-functions and a $p$-adic Poisson kernel. Invent. Math. 101(2), 395-410 (1990)

[Vi] Vignéras, M.F.: Arithmétique des algèbres de quaternions. Lect. Notes Math., vol. 800. Springer, Berlin (1980)

[Wa] Waldspurger, J.-L.: Sur les valeurs de certaines fonctions $L$ automorphes en leur centre de symétrie. Compos. Math. 54(2), 173-242 (1985)

[W] Wiles, A.: Modular elliptic curves and Fermat's Last Theorem. Ann. Math. 141, 443-551 (1995)

[Zh1] Zhang, S.: Heights of Heegner points on Shimura curves. Ann. Math. (2) 153(1), 27-147 (2001)

[Zh2] Zhang, S.: Gross-Zagier formula for GL 2 . Asian J. Math. 5(2), 183-290 (2001) 